\title{
Use of Adjectives in Abstracts when Reporting Results of Randomized, Controlled Trials from Industry and Academia
}

\author{
M. Soledad Cepeda $\cdot$ Jesse A. Berlin $\cdot$ Susan C. Glasser • \\ Wendy P. Battisti $\cdot$ Martijn J. Schuemie
}

Published online: 7 March 2015

(C) The Author(s) 2015. This article is published with open access at Springerlink.com

\begin{abstract}
Objective Accurate representation of study findings is crucial to preserve public trust. The language used to describe results could affect perceptions of the efficacy or safety of interventions. We sought to compare the adjectives used in clinical trial reports of industry-authored and non-industry-authored research.

Methods We included studies in PubMed that were randomized trials and had an abstract. Studies were classified as "non-industry-authored" when all authors had academic or governmental affiliations, or as "industry-authored" when any of the authors had industry affiliations. Abstracts were analyzed using a part-of-speech tagger to identify adjectives. To reduce the risk of false positives, the analysis was restricted to adjectives considered relevant to "coloring" (influencing interpretation) of trial results. Differences between groups were determined using exact tests, stratifying by journal.

Results A total of 306,007 publications met the inclusion criteria. We were able to classify 16,789 abstracts; 9,085 were industry-authored research, and 7,704 were non-industry-authored research. We found a differential use of adjectives between industry-authored and non-industryauthored reports. Adjectives such as "well tolerated" and
\end{abstract}

M. S. Cepeda $(\bowtie)$. W. P. Battisti - M. J. Schuemie

Janssen Research \& Development, LLC, 1125 Trenton

Harbourton Road, Titusville, NJ 08560, USA

e-mail: scepeda@its.jnj.com

\section{J. A. Berlin}

Johnson \& Johnson Chief Medical Office, 410 George Street,

New Brunswick, NJ 08901, USA

S. C. Glasser

Janssen Research \& Development, LLC, 920 Rt 202, Raritan, NJ 08869, USA "meaningful" were more commonly used in the title or conclusion of the abstract by industry authors, while adjectives such as "feasible" were more commonly used by non-industry authors.

Conclusions There are differences in the adjectives used when study findings are described in industry-authored reports compared with non-industry-authored reports. Authors should avoid overusing adjectives that could be inaccurate or result in misperceptions. Editors and peer reviewers should be attentive to the use of adjectives and assess whether the usage is context appropriate.

\section{Key Points}

The language used to describe results could affect perceptions of the efficacy or safety of interventions.

There are differences in the adjectives used when study findings are described in industry-authored reports compared with non-industry-authored reports.

Authors should avoid overusing adjectives that could be inaccurate or result in misperceptions.

Editors and peer reviewers should be attentive to the use of adjectives and assess whether the usage is context appropriate.

\section{Background}

Accurate understanding of the efficacy and safety of health interventions is crucial for public health. Major impediments to such understanding include selective reporting of trial results and inadequate reporting of trial results. 
Publication of only studies that show benefit, known as publication bias, leads to overestimation of the efficacy of interventions. Inadequate reporting of trial results limits the ability of the reader to assess the validity of trial findings $[1,2]$. The CONSORT initiative [3] has led to improvements in the quality of reporting of trial results $[4,5]$. In addition, mandatory registration of clinical trials and mandatory publication of trial results are strategies implemented to diminish the impact of publication bias [6].

How trial results are described in publications may influence the reader's perception of the efficacy and safety of interventions. For example, an intervention can be portrayed as beneficial in the publication despite having failed to differentiate statistically from placebo. In this type of bias, called spin bias, the reader is distracted from the nonsignificant results [7]. The language used to describe trial results could also affect perceptions of the efficacy or safety of health interventions as well as the quality of the study. We studied the vocabulary used to report trial results and compared it between two authorship groups (industry versus non-industry).

\section{Objective}

The objective of this study was to compare the adjectives used to report results of clinical trials between industry and non-industry (academia and government). We focused on adjectives because their use adds "color" (potentially biasing interpretation) to the description of study findings.

\section{Methods}

\subsection{Inclusion Criteria}

We included studies indexed in PubMed that were randomized, controlled trials; assessed humans; had an abstract; and were published in English. The search was conducted on October 7, 2013, without any time limit (all articles present in PubMed until that time). The PubMed query used to identify the studies was "English[lang] AND Randomized Controlled Trial[ptyp] AND humans[MeSH Terms] AND has abstract[text]".

\subsection{Classification of Abstracts}

Studies were classified as industry-authored or non-industry-authored (academia and government), depending on the affiliation of the authors, using an automated algorithm. To determine the affiliation of an author, the affiliation field in PubMed was used to scan for word patterns indicating an industry (e.g., “janssen”, “johnson \& johnson”), academic affiliation (e.g., "university", "school") or government (e.g., "centers? for disease control", "ul\.?s\.? agency"). Because the PubMed affiliation field contains the affiliation of only one of the authors and therefore could not be used as conclusive evidence in papers written by multiple authors, we supplemented the search for the authors' affiliation, using PubMed Central ${ }^{\circledR}$. PubMed Central is a free full-text archive of biomedical journals and therefore lists the affiliations of each one of the authors of a manuscript. Appendix 1 contains the complete list of patterns used for the abstract classification.

For the abstracts not included in PubMed Central, we developed an algorithm to predict the affiliation of the authors. We assumed that if an author had a particular affiliation in one manuscript, that author would also have that affiliation in any other manuscript written by that author in the same year. Because there are no unique identifiers for authors in PubMed, we used an author name disambiguation algorithm similar to Authority [8], which models the probability that two articles sharing the same author name were written by the same individual. The probability was estimated using a random forest [9] classifier using these features as input: length of author name, author name frequency in Medline, similarity in MeSH terms, words in the title or words in the abstract, whether the paper was in the same journal, overlap of other authors, and time between publication in years. The classifier was trained on a set where positive cases were identified using author e-mail addresses (only available for very few authors), and negative controls cases were identified based on mismatch in author first name. The probability was subsequently used in a greedy cluster algorithm to group all papers by an author.

An abstract was classified as non-industry-authored when all authors of the publication had academic or government affiliations. An abstract was classified as industryauthored when any of the authors in the publication had an industry affiliation. Publications in which the algorithm found none of the patterns to classify an author, or found an author with affiliations to both industry and academia or government, were excluded from the analysis.

To assess the accuracy of the algorithm that predicted author affiliation, we selected a random sample of 250 abstracts and manually checked the affiliation of each one of the authors in the full manuscript and compared these results with the algorithm's classification.

\subsection{Adjective Selection}

To compare the use of language between industry and nonindustry authors, we downloaded the Medline database. The abstracts that met the inclusion criteria were run through the part-of-speech tagger of OpenNLP [10], which allowed us to classify the adjectives. OpenNLP uses the Penn Treebank 
tagset, and we considered all tokens with tags JJ (adjective), JJR (adjective, comparative) and JJS (adjective, superlative) [11]. OpenNLP is an open-source machine learning-based toolkit for the processing of natural language text, made available by the Apache Foundation. It has an overall accuracy of around $97 \%$ [12]. We focused on abstracts because more people read the abstract than the whole article, and because only abstracts are available in Medline.

After extracting all adjectives from the abstracts, we selected a set of adjectives we considered relevant to coloring the results of a trial. This selection was performed independently by two authors (MSC and MS), after which discrepancies were resolved in conference. All subsequent analyses were limited to this set to reduce the risk of false positives. Examples of excluded adjectives are "viscous" and "intellectual". The list of adjectives included and excluded is shown in Appendix 2.

\subsection{Location in the Abstract}

To locate where in the abstract the differences in adjective use occurred, we looked separately at the title and conclusion. The title is clearly identified in PubMed records. For unstructured abstracts, the conclusion was considered to be in the last two sentences of the abstract. Sentences were detected using the OpenNLP toolkit [10].

\subsection{Analysis}

To determine whether an adjective was used more often by industry or non-industry authors, we used an exact test for contingency tables [13], stratifying by journal to adjust for any differences in language in the different journals. This test is similar to the well-known Mantel-Haenszel test in that it tests for an overall difference between groups through differences within strata, but it uses an exact method, making it more robust for small numbers within each stratum. We further restricted the adjectives to those that were present in at least 100 papers in our final data set. Because of the large number of tests, we corrected for multiple testing, using Holm's technique [14].

We also calculated a relative estimate. Values $>1$ mean that industry uses that adjective more. Values $<1$ mean that the adjective is used more often by academia and government. A value of exactly 1.0 would indicate equal use by both groups of authors. We report $95 \%$ confidence intervals (CIs), but these intervals are not adjusted for multiple testing.

We further computed the average number of "colored" adjectives used in the title and abstract, where any adjective that was used multiple times in an abstract was counted multiple times. For this analysis, we used all adjectives we considered relevant, including those that appeared in fewer than 100 articles.

\subsection{Source of Funding}

Using authors' affiliation is one way to classify studies as either industry or non-industry. Funding of the study is another way. PubMed identifies financial support of the research, but it would only allow for a US-government and non-US-government funding classification. Sources of financial support are often listed in the full manuscript. For the subset of abstracts that had the full-text articles in PubMed Central, we identified the source of funding and then compared that classification with our affiliation-based classification to provide an estimate of the degree of potential discordance. For example, a trial conducted by an academic institute may be authored by academicians only but funded by a pharmaceutical company. Under the affiliation classification, the research would be considered as non-industry, while under the sponsorship classification, it would be considered as industry. Because the information was only available for a limited number of abstracts, we could not conduct sensitivity analyses, but we are reporting the findings.

\section{Results}

A total of 306,007 publications met the inclusion criteria. We were able to classify 16,789 abstracts; 9,085 were classified as industry, and 7,704 were classified as nonindustry. The algorithm correctly identified 235 of the 250 manuscripts sampled for accuracy (15 were incorrectly assessed as non-industry-authored), indicating that the accuracy of the classifying algorithm was $94 \%$ with a Kappa value of 0.88 (Table 1 ).

The abstracts were published from 1981 to 2013, and $92.5 \%$ were published in 2000 or after. The abstracts were published in 1,788 journals, and $50 \%$ were published in 98 journals. Appendix 3 provides the list of journals, with the number of abstracts by journal.

The 16,789 abstracts had a total of 4,690 adjectives: 298 were considered relevant by both of the authors (see Appendix 4), and 72 adjectives were present in at least 100 papers in our final data set and were analyzed (Table 2). With few exceptions, these were positive adjectives.

The use of adjectives differed between industry and nonindustry (Table 3). Ten adjectives located in the title or conclusion, and 15 adjectives located anywhere in the abstract, had relative use values $>1$, indicating preferential use by industry. Most notably, adjectives such as "well tolerated" and "meaningful" were more commonly used by industry-authored reports in the title or conclusion of the abstracts [relative use 5.20 (CI 2.73-10.03) and 3.08 (CI 1.73-5.44), respectively], whereas adjectives such as "feasible" were more commonly used in abstracts 
Table 1 Assessment of the accuracy of the automated algorithm to classify abstracts as "industry-authored" and "nonindustry-authored", compared with a manual classification, in a subsample of abstracts

Table 2 Adjectives in the final analysis, with the numbers of abstracts in which each adjective appears

\begin{tabular}{lccc}
\hline & \multicolumn{2}{l}{ Manual classification based on full manuscript } \\
\cline { 2 - 4 } & Non-industry-authored & Industry-authored & Total \\
\hline Classification based on automated algorithm & & \\
Non-industry-authored & 113 & 0 & 113 \\
Industry-authored & 15 & 122 & 137 \\
Total & 128 & 122 & 250 \\
\hline
\end{tabular}

\begin{tabular}{|c|c|c|c|c|c|}
\hline Adjective & Industry use & Non-industry use & Adjective & Industry use & Non-industry use \\
\hline Acceptable & 195 & 120 & Meaningful & 285 & 60 \\
\hline Adequate & 116 & 125 & Mild & 732 & 211 \\
\hline Apparent & 274 & 76 & Mild-to-moderate & 110 & 21 \\
\hline Appropriate & 165 & 190 & Minor & 132 & 93 \\
\hline Comparable & 699 & 300 & Moderate & 715 & 385 \\
\hline Complete & 267 & 226 & Modest & 164 & 93 \\
\hline Comprehensive & 50 & 125 & Necessary & 131 & 164 \\
\hline Considerable & 44 & 84 & Novel & 179 & 77 \\
\hline Consistent & 608 & 196 & Poor & 185 & 286 \\
\hline Conventional & 227 & 310 & Potent & 383 & 52 \\
\hline Cost-effective & 55 & 143 & Promising & 82 & 158 \\
\hline Detectable & 68 & 36 & Protective & 96 & 96 \\
\hline Difficult & 69 & 154 & Relevant & 458 & 247 \\
\hline Effective & 1,345 & 1,332 & Reliable & 73 & 95 \\
\hline Efficacious & 239 & 108 & Responsible & 45 & 56 \\
\hline Efficient & 48 & 96 & Robust & 86 & 69 \\
\hline Enhanced & 81 & 108 & Safe & 547 & 291 \\
\hline Equal & 114 & 110 & Serious & 723 & 194 \\
\hline Essential & 101 & 106 & Severe & 741 & 393 \\
\hline Evidence-based & 20 & 135 & Significant & 3,238 & 2,809 \\
\hline Evident & 91 & 94 & Similar & 2,405 & 997 \\
\hline Excellent & 73 & 67 & Simple & 58 & 171 \\
\hline Extensive & 77 & 53 & Strong & 116 & 155 \\
\hline Fast & 77 & 28 & Stronger & 34 & 74 \\
\hline Faster & 66 & 45 & Substantial & 180 & 157 \\
\hline Favorable & 196 & 83 & Successful & 83 & 254 \\
\hline Favourable & 73 & 37 & Sufficient & 65 & 101 \\
\hline Feasible & 43 & 167 & Traditional & 62 & 153 \\
\hline Good & 309 & 253 & Unchanged & 236 & 101 \\
\hline Important & 539 & 730 & Unexpected & 100 & 29 \\
\hline Improved & 176 & 149 & Unlikely & 105 & 41 \\
\hline Inadequate & 145 & 45 & Useful & 180 & 252 \\
\hline Inferior & 40 & 62 & Usual & 131 & 587 \\
\hline Intensive & 99 & 246 & Valid & 64 & 60 \\
\hline Long-term & 590 & 507 & Validated & 151 & 84 \\
\hline Major & 596 & 524 & Well tolerated & 249 & 38 \\
\hline
\end{tabular}

classified as non-industry-authored [relative use 0.34 (CI 0.18-0.6)]. Adjectives such as "successful" and "usual" were also more commonly used by non-industry, when considering the abstract overall [relative use 0.46 (CI 0.31-0.68) and 0.40 (CI $0.30-0.53$ ), respectively] (Table 3). 
Table 3 Adjectives favored by industry or non-industry (academia and government) by location in the abstract

\begin{tabular}{|c|c|c|}
\hline \multirow[t]{2}{*}{ Adjective } & \multicolumn{2}{|c|}{ Relative use (95\% confidence interval) } \\
\hline & Title or conclusion & Entire abstract including title \\
\hline Well tolerated & $5.20(2.73-10.03)$ & $4.96(2.99-8.22)$ \\
\hline Meaningful & $3.08(1.73-5.44)$ & $3.42(2.24-5.21)$ \\
\hline Efficacious & $2.78(1.81-4.24)$ & $1.96(1.40-2.74)$ \\
\hline Consistent & $2.49(1.74-3.53)$ & $2.21(1.72-2.83)$ \\
\hline Mild & $2.40(1.60-3.57)$ & $2.91(2.28-3.70)$ \\
\hline Good & $2.37(1.56-3.56)$ & \\
\hline Severe & $2.06(1.51-2.79)$ & $1.82(1.49-2.21)$ \\
\hline Comparable & $1.98(1.41-2.76)$ & $1.92(1.55-2.36)$ \\
\hline Similar & $1.98(1.60-2.45)$ & $1.89(1.67-2.13)$ \\
\hline Significant & $1.52(1.28-1.79)$ & \\
\hline Feasible & $0.34(0.18-0.60)$ & $0.40(0.23-0.66)$ \\
\hline Serious & & $3.29(2.53-4.26)$ \\
\hline Inadequate & & $2.84(1.66-4.79)$ \\
\hline Potent & & $2.26(1.48-3.445)$ \\
\hline Acceptable & & $1.96(1.35-2.80)$ \\
\hline Relevant & & $1.67(1.27-2.16)$ \\
\hline Moderate & & $1.62(1.33-1.97)$ \\
\hline Safe & & $1.58(1.25-1.98)$ \\
\hline Successful & & $0.46(0.31-0.68)$ \\
\hline Usual & & $0.40(0.30-0.53)$ \\
\hline
\end{tabular}

The numbers represent a relative estimate: $>1$ means favored by industry; $<1$ means favored by non-industry. The adjectives shown in bold text are more commonly used by non-industry authors

All of the adjectives in this table are statistically significant between industry and non-industry after adjustment for multiple testing

Confidence intervals are not adjusted for multiple testing

Results are stratified by journal to adjust for differences in language in the different journals

Examples of the contexts in which the adjectives were used in the title or conclusion of the abstract are presented in Table 4.

On average, there were 2.6 "colored" adjectives in each abstract, and this number was the same for both industryauthored and non-industry-authored research.

\subsection{Source of Funding}

When we estimated the degree of potential discordance between abstracts classified by author affiliation or by source of funding, we found that of the 16,789 abstracts that we could classify as industry-authored or non-industryauthored research, only $189(1.1 \%)$ had the full text available in PubMed Central and disclosed either partial or total funding by industry; $16 \%$ of these studies were classified as being from non-industry when looking at authors' affiliations.
Table 4 Selected examples of the context of adjective use in the abstract

"The data suggest that enalapril is efficacious and well tolerated by the high-risk patients" [Industry-authored]

"Patients with severe asthma who received FP [at approximately half the dose of BDP/BUD] experienced statistically significant as well as clinically meaningful, improvements in their HRQoL" [Industry-authored]

"Evaluation of pre-discharge home visits by occupational therapists in a rehabilitation setting is feasible, but a more effective recruitment strategy for a main study is favored by application of a multi-centre setting" [Non-industry-authored]

"All treatment regimens were well tolerated; drug-related adverse reaction rates were similar across the 3 groups" [Industryauthored]

"Adalimumab is the first TNF antagonist to demonstrate good clinical efficacy and safety in patients with axial SpA without radiographically defined sacroiliitis" [Industry-authored]

"This simple, feasible and inexpensive bioassay may promote the development of models more predictive of microbicide efficacy" [Non-industry-authored]

"Successful treatment of tobacco dependence in respiratory patients requires repeated intensive interventions" [Nonindustry-authored]

\section{Discussion}

There are differences in the adjectives used when study findings are described in industry-authored compared with non-industry-authored reports. Certain adjectives are five times more commonly used by industry, although, on average, both groups of authors use the same number of "colored" adjectives.

The differences in the adjectives used that were noted in the present study support anecdotal evidence about the way results of clinical trials are reported by industry. The Medical Publishing Insights and Practices (MPIP) initiative in 2012 recommended avoiding broad statements such as "generally safe and well tolerated" [15] when reporting trial resultsprecisely the type of adjectives we found were more commonly used by industry. The MPIP initiative was founded by members of the pharmaceutical industry and the International Society for Medical Publication Professionals to elevate trust, transparency, and integrity in publishing industrysponsored studies. Describing an intervention as "well tolerated", which is the adjective with the largest use in industry-authored manuscripts compared with academic or government-authored manuscripts-although accurate in certain circumstances, considering the nature of the trials conducted by industry-might not be generalizable to the broader population when the trial is small, when relatively "healthy" or "stable" participants are recruited (compared with the broader population with the target indication), or when the follow-up is short. 
The use of those adjectives (such as "acceptable", "meaningful", "potent", or "safe") more commonly present in industry-authored reports than in non-industry-authored reports could suggest that industry-authored reports tend to focus on the positive aspects of the health intervention being evaluated. However, the differences in adjective use could also reflect variations in the types of trials conducted by industry and academic or governmental institutions. Industry studies tend to focus on drugs or devices, while non-industry work is likely to be more inclusive of other types of heath interventions. By controlling for journal, we adjusted partially for potential differences in the studies.

We used the affiliation of the authors to classify publications as industry-authored and non-industry-authored. Even if one author out of many was from industry, the paper was classified as industry-authored. Although this approach could seem extreme, it has also been recommended by others [16]. It might seem preferable to look at the source of funding instead of the author affiliation; however, this approach too has its shortcomings. Funding mechanisms are complex, some journals do not report funding sources, full reporting of all sources of financial support is not complete, and there are different levels of support, from unrestricted educational grants to support that includes input of the manufacturers into trial designs, conduct of the analysis and publication [16, 17]. We assessed how many of the abstracts in which the full manuscript reported support from industry were classified as being non-industry in origin. We found that $16 \%$ of those papers with industry support were classified as being from non-industry. It is difficult to predict the direction of bias related to any potential misclassification because of the shortcomings listed above, but in the worst-case scenario, it would lead to an underestimation of the relative measure and consequently to a smaller set of adjectives because of loss of power.

The results of this study are based on assessing the abstract instead of the full paper. Many readers just read the abstract of the published article and may be influenced by it, so we argue that it is an important place to look for differences in reporting style. The results of a study that assessed the impact of "spin" on interpretation of cancer trials showed that clinicians' interpretation was affected by reading the abstracts of the trials [18]. In addition, the study focused on randomized, controlled trials, thus the findings may not apply to other types of study designs.

The present study was limited to counting adjectives and assessing the difference in those counts in industry-authored and non-industry-authored reports. Although we have provided some examples to illustrate how they were used, the study ignored the context of the usage. A thematic analysis could allow detection of patterns, and a critical review of the full text of the paper could determine whether the use of the adjectives was indeed appropriate in view of the data or what it is known in the field.

\section{Conclusion}

We assessed a very large number of abstracts and found differences in how study findings are described by industry-affiliated authors as compared with non-industry-affiliated authors. The language used to describe trial results could affect perceptions of the efficacy or safety of health interventions. Authors should avoid overusing adjectives that could be inaccurate or potentially lead to misperceptions. Editors and peer reviewers should be attentive to the use of adjectives in the abstract (and the manuscript in its entirety) and assess whether their use is appropriate.

Acknowledgments and declaration of competing interests All authors are employees of Janssen Research \& Development, LLC, except for Jesse A. Berlin, who is an employee of Johnson \& Johnson, the parent company of Janssen Research \& Development, LLC.

Author Contributions MSC: study conception and design, interpretation of study findings, manuscript preparation.

JAB: interpretation of study findings, revising manuscript critically for important intellectual content.

SCG: interpretation of study findings, revising manuscript critically for important intellectual content.

WPB: interpretation of study findings, revising manuscript critically for important intellectual content.

MS: study design and execution, analysis, interpretation of study findings, manuscript preparation.

All authors read and approved the final manuscript.

Open Access This article is distributed under the terms of the Creative Commons Attribution Noncommercial License which permits any noncommercial use, distribution, and reproduction in any medium, provided the original author(s) and the source are credited.

\section{Appendix 1: Industry and academic/governmental affiliation patterns}

\begin{tabular}{llll}
\hline $\begin{array}{l}\text { Industry } \\
\text { patterns }\end{array}$ & $\begin{array}{l}\text { Industry } \\
\text { patterns }\end{array}$ & $\begin{array}{l}\text { Academic/ } \\
\text { government } \\
\text { patterns }\end{array}$ & $\begin{array}{l}\text { Academic/ } \\
\text { government } \\
\text { patterns }\end{array}$ \\
\hline Abbott & Merz & Clinic & School \\
Amgen & Mundipharma & Clinique & Sjukhuset \\
Astrazeneca & Nitec & College & Universidad \\
Aventis & Nordis & Ecole & Universidade \\
Bayer & Novartis & Foundation & Universitaires \\
Boehringer & Pfizer & Gasthuis & Universitária \\
Centocor & Pharmaceuticals & Hôpital & Universitat \\
Genentech & Procter & Hospital & Universite \\
Glaxo & Sanofi & Hospitalario & Université \\
\hline
\end{tabular}




\begin{tabular}{|c|c|c|c|}
\hline $\begin{array}{l}\text { Industry } \\
\text { patterns }\end{array}$ & $\begin{array}{l}\text { Industry } \\
\text { patterns }\end{array}$ & $\begin{array}{l}\text { Academic/ } \\
\text { government } \\
\text { patterns }\end{array}$ & $\begin{array}{l}\text { Academic/ } \\
\text { government } \\
\text { patterns }\end{array}$ \\
\hline Glaxosmithkline & Shionogi squibb & Hospitales & Universiteit \\
\hline Gsk & Takeda & Hospitalier & Universiti \\
\hline Humax & Ucb & Hospitaller & University \\
\hline Janssen & Vifor & Karolinska & Ziekenhuis \\
\hline Kaken & Zymogenetics & Kliniek & $\begin{array}{l}\text { Centers? for } \\
\text { disease } \\
\text { control }\end{array}$ \\
\hline Lilly & $\begin{array}{l}\text { Industrie } \\
\text { farmaceutiche }\end{array}$ & Kliniken & Centro de salud \\
\hline Mcneil & $\begin{array}{l}\text { Johnson (\&land) } \\
\text { johnson }\end{array}$ & Klinikum & Centro médico \\
\hline Medimmune & $\begin{array}{l}\text { Pharmaceutical } \\
\text { company }\end{array}$ & Krankenhaus & $\begin{array}{l}\text { Memorial sloan } \\
\text { kettering } \\
\text { cancer center }\end{array}$ \\
\hline \multirow[t]{4}{*}{ Medtronic } & $\begin{array}{l}\left(\left[{ }^{\wedge} \mathrm{a}\right]\left[\left[^{\wedge} \mathrm{a}-\right.\right.\right. \\
\left.\mathrm{z}]^{\wedge}\right) \operatorname{roche}\left(\left[{ }^{\wedge} \mathrm{a}-\right.\right. \\
\mathrm{z}] \mid \$)\end{array}$ & National & ul\.?s\\
.? agency \\
\hline & & Nazionale & $\begin{array}{l}\text { Health } \\
\text { department }\end{array}$ \\
\hline & & Policlinico & $\begin{array}{l}\text { Food and drug } \\
\text { administration }\end{array}$ \\
\hline & & Polyclinique & \\
\hline
\end{tabular}

Patterns with symbols represent standard regular expressions (a pattern used to search for text)

\section{Appendix 2: All adjectives present in the analyzed abstracts}

\begin{tabular}{|c|c|}
\hline $1000-\mathrm{mg}$ & 10-year \\
\hline $100-\mathrm{mg}$ & 11-day \\
\hline $100-\mathrm{mm}$ & 11-fold \\
\hline 100-point & 11-point \\
\hline $10-\mathrm{cm}$ & 11-week \\
\hline 10-day & $120-\mathrm{mg}$ \\
\hline 10-fold & 12-day \\
\hline $10-\mathrm{h}$ & $12-h$ \\
\hline 10 -item & 12-hour \\
\hline $10 \mathrm{mg}$ & 12-item \\
\hline $10-\mathrm{mg}$ & 12-lead \\
\hline $10-\min$ & $12-\mathrm{mg}$ \\
\hline 10-minute & 12-month \\
\hline $10-\mathrm{mm}$ & 12-months \\
\hline 10-month & 12 th \\
\hline 10-point & 12-week \\
\hline 10 th & $12-w k$ \\
\hline 10 -valent & 13-week \\
\hline 10-week & 14-day \\
\hline
\end{tabular}

14-week

150-mg

15-day

15-item

$15-\min$

15-minute

15-month

15 th

15-year

16-week

17beta-estradiol

17-item

18-month

18-week

$1 \mathrm{~b}$

$1 \mathrm{c}$

1-compartment

1-day

1-g

1-h

1-hour

1-infected

1-mg

1-month

1-point

$1 \mathrm{rm}$

$1 \mathrm{~s}$

1-sided

1 st

1-week

1-year

$1-\mathrm{yr}$

$200 \mathrm{mg}$

200-mg

20-item

$20 \mathrm{mg}$

20-mg

20-min

20-minute

20-month

20-week

21-day

21-item

$23 \mathrm{f}$

23-valent

$24 \mathrm{~h}$

24-h

24-hour

24-hr

24-month

24-months

24-week

250-mg 25-hydroxyvitamin

25-item

25-mg

25 th

26-week

28-day

28-item

28-joint

28-week

2-arm

2-compartment

$2 \mathrm{~d}$

2-day

2-dose

2-fold

2-g

2-h

2-hour

2-hr

$2 \max$

2-mg

2-minute

2-month

2-part

2-period

2-phase

2-point

2-sequence

2-sided

2-stage

2-way

2-week

2-wk

2-year

$2-y r$

300-mg

30-day

30-fold

30-item

30-mg

30-min

30-minute

30-month

30-second

30-week

32-week

35-day

36-item

36-week

3-arm

$3 d$

3-d

3-day 


\begin{tabular}{|c|c|c|c|}
\hline 3-dimensional & 5-fold & 80-mg & acid \\
\hline 3 -dose & $5-f u$ & 85 th & acidic \\
\hline 3-drug & 5-hmt & 8-day & acid-related \\
\hline 3-fold & 5-ht & 8-fold & acid-suppressive \\
\hline $3-h$ & 5-ht3 & $8-h$ & acne \\
\hline 3-hour & 5-httlpr & 8-hour & acoustic \\
\hline 3-hydroxy-3- & 5-hydroxyindoleacetic & $8-m g$ & acr20 \\
\hline methylglutaryl & 5-hydroxymethyl & 8-month & acromegaly \\
\hline $3 \mathrm{mg}$ & 5-hydroxytryptamine & 8th & acting \\
\hline $3-\mathrm{mg}$ & 5-lipoxygenase & 8-week & actinic \\
\hline 3-month & $5 \mathrm{mg}$ & 90-day & activate \\
\hline 3-months & $5-m g$ & 90-minute & activated \\
\hline 3-period & 5-minute & 90th & active \\
\hline $3 \mathrm{rd}$ & 5-month & 95 th & active-comparator- \\
\hline 3-treatment & 5-period & 96-week & controlled \\
\hline 3-way & 5-point & 9-day & active-control \\
\hline 3-week & 5 th & 9-month & active-controlled \\
\hline 3-weekly & 5-way & 9-week & activity-related \\
\hline $3-w k$ & 5-week & $\mu \mathrm{g}$ & actual \\
\hline 3-year & 5-year & $\mu$-opioid & acute \\
\hline $400 \mathrm{mg}$ & 5-year-old & a1c & acutely \\
\hline 400-mg & $5-y r$ & abacavir & acute-phase \\
\hline $40-\mathrm{mg}$ & $600-\mathrm{mg}$ & abatacept-treated & adalimumab \\
\hline 40-week & 60-mg & abbreviated & adalimumab-treated \\
\hline 45-min & $60-\min$ & abciximab & adaptive \\
\hline 46-week & 6-day & abdominal & adas-cog \\
\hline $48-h$ & $6-h$ & aberrant & added \\
\hline 48-hour & 6-hour & abiraterone & additional \\
\hline 48-week & 6-min & able & additive \\
\hline 4-day & 6-minute & abnormal & add-on \\
\hline 4-fold & 6-month & aboriginal & adenosine \\
\hline $4-h$ & 6-months & above & adequate \\
\hline 4-hour & $6 \mathrm{mwt}$ & abrupt & adherent \\
\hline 4-mg & 6-point & absent & adhesive \\
\hline 4-month & 6th & absolute & adipose \\
\hline 4-period & 6-week & absorbable & adjacent \\
\hline 4-point & $6-w k$ & abstinent & adjudicated \\
\hline 4th & 6-year & abstract & adjunct \\
\hline 4-way & $70-\mathrm{kg}$ & abused & adjunctive \\
\hline 4-week & 70-mg & academic & adjustable \\
\hline 4-weeks & $72-\mathrm{h}$ & acarbose & adjusted \\
\hline 4-wk & 72-hour & acceptable & adjuvant \\
\hline 4-year & $750-\mathrm{mg}$ & accepted & adjuvanted \\
\hline $4-y r$ & $75-g$ & accessible & administrative \\
\hline 500-mg & 75-mg & accidental & adolescent \\
\hline $50 \mathrm{mg}$ & 75 th & accompanying & adp-induced \\
\hline $50-\mathrm{mg}$ & 7-day & accurate & adrenal \\
\hline 50-week & 7-point & acellular & adrenergic \\
\hline 52-week & 7-valent & acetabular & adult \\
\hline 54-week & 7verm & acetic & advanced \\
\hline 5alpha-reductase & 7-week & acetylsalicylic & advantageous \\
\hline 5-day & 7-year & achievable & adverse \\
\hline 5-fluorouracil & 800-mg & achieved & advisable \\
\hline
\end{tabular}




\begin{tabular}{|c|c|c|c|}
\hline aerobic & ambulatory & anthropometric & antioxidative \\
\hline aerosol & ameliorate & anti-aging & antiparkinsonian \\
\hline aerosolized & amenable & anti-ama1 & antiplaque \\
\hline aesthetic & american & antianginal & anti-plaque \\
\hline afebrile & amg & anti-angiogenic & antiplatelet \\
\hline affected & amino & antiarrhythmic & anti-platelet \\
\hline affective & amino-terminal & anti-atherogenic & anti-prp \\
\hline affordable & aminotransferase & antibacterial & antipsychotic \\
\hline aforementioned & amiodarone & antibiotic & antipyretic \\
\hline african & amlodipine & anticalculus & antipyrine \\
\hline african-american & amodiaquine & anticholinergic & antiresorptive \\
\hline age-adjusted & amplitude & anticipated & antiretroviral \\
\hline age-appropriate & amprenavir & anticipatory & anti-retroviral \\
\hline aged & amyloid & anti-circumsporozoite & antiretroviral-experienced \\
\hline age-dependent & anabolic & anticoagulant & antiretroviral-naïve \\
\hline age-matched & anaemic & antidepressant & antiretroviral-naive \\
\hline age-related & anaerobic & antidepressive & antirheumatic \\
\hline age-specific & anaesthetic & antidiabetic & anti-rheumatic \\
\hline aggregate & analgesic & anti-diabetic & antisecretory \\
\hline aggressive & analogous & antiemetic & antisense \\
\hline aging & analysed & antiepileptic & antiseptic \\
\hline agitated & analytic & anti-factor & antithrombin \\
\hline agonist & analytical & antifracture & antithrombotic \\
\hline ai & anamnestic & antifungal & anti-tnf \\
\hline$a-i$ & anastomotic & antigen & anti-tnf-alpha \\
\hline air & anastrozole & antigenic & antitumor \\
\hline airborne & anatomic & antigen-specific & anti-tumor \\
\hline al & anatomical & anti-hav & antitumour \\
\hline alanine & anchor-based & anti-hbs & anti-tumour \\
\hline alcohol-dependent & ancillary & anti-hcv & anti-vegf \\
\hline alcohol-free & anecdotal & antihistamine & antiviral \\
\hline alcoholic & anemic & anti-hiv & anti-xa \\
\hline alcohol-related & anesthetic & anti-hpv & antral \\
\hline aldosterone & anesthetized & anti-hpv-16 & anxiolytic \\
\hline alendronate & aneurysmal & anti-human & anxious \\
\hline alert & anginal & antihyperglycaemic & aortic \\
\hline alive & angiogenic & antihyperglycemic & aphthous \\
\hline all-cause & angiographic & antihypertensive & apical \\
\hline allele & angioplasty & anti-hypertensive & apoa-i \\
\hline allelic & angiotensin-converting & anti-ige & apolipoprotein \\
\hline allergen-specific & angry & antiinflammatory & apoptotic \\
\hline allergic & angular & anti-inflammatory & apparent \\
\hline allometric & animal & anti-influenza & applicable \\
\hline allosteric & ankle-brachial & 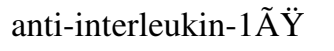 & appreciable \\
\hline all-patients-treated & ankylosing & antimalarial & appropriate \\
\hline alone & annual & anti-malarial & approved \\
\hline alpha-linolenic & annular & antimicrobial & approximate \\
\hline altered & antacid & antimigraine & aprepitant \\
\hline alternate & antagonistic & antimuscarinic & aqueous \\
\hline alternative & antecedent & antimycobacterial & arachidonic \\
\hline alveolar & antenatal & antineoplastic & arbitrary \\
\hline ambient & anterior & antinociceptive & ar-c124910xx \\
\hline ambiguous & anteroposterior & antioxidant & arginine-stimulated \\
\hline
\end{tabular}




\begin{tabular}{|c|c|c|c|}
\hline aripiprazole & atopic & base & biopsy-confirmed \\
\hline aripiprazole-treated & atorvastatin & baseline & biopsy-proven \\
\hline arithmetic & atp-binding & baseline-adjusted & bioresorbable \\
\hline armed & atrial & baseline-corrected & biphasic \\
\hline aromatase & atrioventricular & baseline-to-endpoint & bipolar \\
\hline arousal & at-risk & basic & bis-guided \\
\hline arrhythmic & atrophic & b-cell & bispectral \\
\hline artemether-lumefantrine & attentional & bcr-abl & bisphosphonate \\
\hline artemisinin-based & attenuated & bdi-ii & bivalent \\
\hline arterial & attractive & behavioral & bivariate \\
\hline arteriovenous & attributable & behavioural & biventricular \\
\hline artesunate & atypical & belgian & biweekly \\
\hline artesunate-amodiaquine & auc & beneficial & bi-weekly \\
\hline arthroplasty & auc0-t & benign & black \\
\hline arthroscopic & audio & best & bladder-related \\
\hline articular & auditory & best-corrected & blend-a-med \\
\hline artificial & augmented & beta & blended \\
\hline as03a-adjuvanted & auricular & beta- $1 b$ & blind \\
\hline as03-adjuvanted & australian & beta2-agonist & blinded \\
\hline as04-adjuvanted & autobiographical & beta-adrenergic & blinded-endpoint \\
\hline ascending-dose & autogenous & beta-agonist & blinding \\
\hline ascorbic & autoimmune & beta-carotene & blue \\
\hline asenapine & autologous & beta-cell & blunt \\
\hline aseptic & automated & beta-haemolytic & blunted \\
\hline asexual & automatic & better & blurred \\
\hline asian & autonomic & between-patient & bodily \\
\hline as-needed & autonomous & between-subject & body \\
\hline aspirate & available & bg & bolus \\
\hline aspirin & average & bi & bone-specific \\
\hline aspirin-treated & aversive & bid & bony \\
\hline assertive & avian & bidirectional & borderline \\
\hline assessable & awake & biexponential & botanical \\
\hline assessor-blind & awakening & big & bound \\
\hline assessor-blinded & aware & bilateral & bovine \\
\hline assigned & axial & biliary & bp-lowering \\
\hline assisted & $\tilde{\mathrm{A}} \ddot{\mathrm{Y}}$ & bilingual & brachial \\
\hline associated & $\tilde{A} \ddot{Y} 2$-agonist & bimanual & brain-derived \\
\hline associative & $\tilde{A} \ddot{Y}$-cell & b-inactivated & branded \\
\hline asthma & azimilide & binary & brazilian \\
\hline asthma-related & azithromycin & binding & breast-fed \\
\hline asthma-specific & azole & binding-adjusted & brief \\
\hline asthmatic & back & binocular & bright \\
\hline as-treated & backward & binomial & brisk \\
\hline asymmetric & bacterial & bioactive & british \\
\hline asymptomatic & bactericidal & bioavailable & broad \\
\hline at & bacteriologic & biochemical & broader \\
\hline atenolol-based & bacteriological & bioelectrical & broad-spectrum \\
\hline atherogenic & bad & bioequivalent & bronchial \\
\hline atherosclerotic & balanced & biologic & bronchoalveolar \\
\hline atherothrombotic & bare & biological & bronchodilatory \\
\hline athletic & baroreflex & biomechanical & bronchopulmonary \\
\hline atomoxetine & basal & biomedical & bronchoscopic \\
\hline atomoxetine-treated & basal-bolus & biopsy & brown \\
\hline
\end{tabular}




\begin{tabular}{|c|c|c|c|}
\hline buccal & cefuroxime & cirrhotic & colony-forming \\
\hline budesonide & celecoxib & cisplatin & colony-stimulating \\
\hline buffered & cell-derived & cisplatin-induced & colorectal \\
\hline buoyant & cell-mediated & citrate & colposcopic \\
\hline bupivacaine & cellular & citric & combined \\
\hline buspirone & cemented & c-kit & comfortable \\
\hline busy & cementless & clarithromycin & commercial \\
\hline $\mathrm{ca}$ & central & classic & common \\
\hline caesarean & centralised & classical & commonest \\
\hline caffeinated & centralized & classified & community-acquired \\
\hline caffeine & cephalic & clean & community-based \\
\hline calculated & ceramic & cleansing & community-dwelling \\
\hline calibrated & cerebral & clear & community-living \\
\hline caloric & cerebrospinal & clearer & comorbid \\
\hline canadian & cerebrovascular & clinical & co-morbid \\
\hline cancer-related & cerivastatin & clinic-based & comparable \\
\hline cancer-specific & certain & clinician & comparative \\
\hline cannabinoid & certolizumab & clinician-administered & comparator-controlled \\
\hline capable & certoparin & clinician-rated & comparator-treated \\
\hline capsaicin-induced & cervical & clonal & compartmental \\
\hline carbohydrate & cervicovaginal & clonidine & compatible \\
\hline carbonic & cesarean & clopidogrel & compelling \\
\hline carcinoembryonic & cetuximab & clopidogrel-treated & compensated \\
\hline cardiac & cgi-i & close & compensatory \\
\hline cardinal & challenging & closed & competent \\
\hline cardiometabolic & characteristic & closed-loop & competitive \\
\hline cardioprotective & chd & closer & complementary \\
\hline cardiopulmonary & cheaper & closest & complete \\
\hline cardiorespiratory & chemical & clustered & complex \\
\hline cardio-respiratory & chemotherapeutic & cluster-randomised & compliant \\
\hline cardiovascular & chemotherapy-induced & cluster-randomized & complicated \\
\hline care-as-usual & chemotherapy-naive & $\mathrm{cm}$ & component \\
\hline care-based & chewable & $\operatorname{cmax}$ & composite \\
\hline careful & chi & $\operatorname{co} 2$ & comprehensive \\
\hline carer & child-pugh & coadministered & compressed \\
\hline carious & chimeric & co-administered & comprised \\
\hline carotid & chinese & coated & compromised \\
\hline carpal & chiropractic & cochran-mantel-haenszel & compulsive \\
\hline carvedilol & chi-square & coefficient & compulsory \\
\hline castle & chi-squared & cognitive & computational \\
\hline castration-resistant & chloroquine & cognitive-behavioral & computed \\
\hline casual & cholesterol-lowering & cognitive-behavioural & computer-assisted \\
\hline catabolic & cholinergic & coherent & computer-based \\
\hline categorical & choroidal & cohort & computer-generated \\
\hline catheter-related & chronic & cold & computerised \\
\hline cationic & $\mathrm{ci}$ & cold-adapted & computerized \\
\hline caucasian & cilomilast & colic & computer-tailored \\
\hline causal & cimetidine & collaborative & concentrated \\
\hline causative & ciprofloxacin & collagen-induced & concentration-dependent \\
\hline cause-specific & circadian & collective & concentration-effect \\
\hline ccr5-tropic & circular & colloid & concentration-time \\
\hline cecal & circumferential & colonic & concentration-versus-time \\
\hline cefaclor-treated & circumsporozoite & colonoscopy & concentric \\
\hline
\end{tabular}




\begin{tabular}{|c|c|c|c|}
\hline conceptual & coprimary & culture-positive & deficient \\
\hline concerned & co-primary & cumulative & definite \\
\hline conclusive & core & curative & definitive \\
\hline concomitant & corneal & current & degenerative \\
\hline concrete & coronal & customary & delayed \\
\hline concurrent & coronary & customized & deleterious \\
\hline conditional & correct & cutaneous & deliberate \\
\hline condition-specific & corrective & cut-off & delta \\
\hline confident & correlate & $\mathrm{cv}$ & deltoid \\
\hline confirmative & correlated & cyclic & delusional \\
\hline confirmatory & corresponding & cycling & demographic \\
\hline confirmed & cortical & cyclooxygenase- 2 & demonstrable \\
\hline conflicting & corticospinal & cyclo-oxygenase-2 & dendritic \\
\hline confounding & corticosteroid & cyclophosphamide & denosumab-treated \\
\hline congenital & cortisol & cyclosporine & dense \\
\hline congestive & cosmetic & cyp & dental \\
\hline congruent & cost & cyp3a & dentinal \\
\hline conjugate & cost-effective & cystatin & dependant \\
\hline conjugated & costly & cystic & dependent \\
\hline conjunctival & cost-saving & cytogenetic & depressant \\
\hline connective & counterbalanced & cytokine & depressed \\
\hline conscious & covariate & cytological & depression-related \\
\hline consecutive & $\operatorname{cox}-2$ & cytoplasmic & depressive \\
\hline consensus-based & c-peptide & cytotoxic & derivative \\
\hline consenting & cranial & $\mathrm{d}$ & dermal \\
\hline consequent & c-reactive & dabigatran & dermatological \\
\hline conservative & creatine & daily & dermatology-specific \\
\hline considerable & creatinine & d-amphetamine & descriptive \\
\hline consistent & creative & danish & design \\
\hline constant & credible & dapagliflozin & desirable \\
\hline constitutional & crevicular & dapoxetine & desloratadine \\
\hline constraint-induced & critical & daptomycin & desvenlafaxine \\
\hline contemporary & cross-clade & darifenacin & detailed \\
\hline content & cross-cultural & dark & detectable \\
\hline contextual & cross-linked & data-driven & detrimental \\
\hline contingent & crossover & day-1 & devastating \\
\hline continual & cross-over & daytime & developed \\
\hline continued & cross-reactive & day-to-day & developmental \\
\hline continuous & cross-sectional & $\mathrm{dbp}$ & devoid \\
\hline contraceptive & crp & d-conjugate & dexlansoprazole \\
\hline contradictory & crucial & d-dimer & dextromethorphan \\
\hline contralateral & cruciate & debatable & diabetes-related \\
\hline contrary & crude & debilitating & diabetes-specific \\
\hline contrast-enhanced & crushed & decaffeinated & diabetic \\
\hline controlled & crystalloid & deceased & diagnosed \\
\hline controversial & $\mathrm{ct}$ & decisional & diagnostic \\
\hline convenient & c-terminal & decision-making & diaphyseal \\
\hline conventional & ctx-ii & declarative & diary \\
\hline convergent & cubic & decompensated & diastolic \\
\hline convincing & cued & decreased & dichotomous \\
\hline cooperative & cultural & deep & didactic \\
\hline coordinated & culture-confirmed & deeper & dietary \\
\hline copd & cultured & defective & diet-induced \\
\hline
\end{tabular}




\begin{tabular}{|c|c|c|c|}
\hline diet-treated & distinctive & dry & eight-week \\
\hline different & distressing & dsm-iii-r & eighty \\
\hline differential & distribution-based & dsm-iv & elastic \\
\hline differentiate & disturbed & dsm-iv-defined & elderly \\
\hline differentiated & diuretic & dsm-iv-tr & e-learning \\
\hline differing & diurnal & dsm-iv-tr-defined & elective \\
\hline difficile & divergent & dual & electric \\
\hline difficult & diverse & dual-chamber & electrical \\
\hline difficult-to-treat & dlqi & dual-energy & electrocardiographic \\
\hline diffuse & docetaxel & dual-therapy & electrochemical \\
\hline digestive & docosahexaenoic & ductal & electroconvulsive \\
\hline digit & doctor-patient & due & electroencephalographic \\
\hline digital & domestic & duloxetine & electrolyte \\
\hline digoxin & dominant & duloxetine-treated & electromagnetic \\
\hline dihydroartemisinin- & donepezil & duodenal & electromyographic \\
\hline piperaquine & dopamine & durable & electronic \\
\hline dilated & dopaminergic & dutasteride & electrophysiological \\
\hline diminished & dorsal & dutch & elemental \\
\hline diphtheria-tetanus-acellular & dorsolateral & dyadic & elementary \\
\hline dipropionate & dorzolamide & dye & eletriptan \\
\hline direct & dorzolamide-timolol & dynamic & elevated \\
\hline direct-acting & dose-adjusted & dysfunctional & elicited \\
\hline directional & dose-dependent & dyslipidemic & eligible \\
\hline dirithromycin-treated & dose-effect & dyspeptic & elispot \\
\hline disabled & dose-escalating & dysphoric & elusive \\
\hline disabling & dose-finding & $\mathrm{e} 2$ & e-mail \\
\hline disadvantaged & dose-independent & earlier & embedded \\
\hline disappointing & dose-limiting & earliest & embolic \\
\hline discernible & dose-linear & early & emergent \\
\hline discontinued & dose-normalized & early-onset & emetic \\
\hline discordant & dose-proportional & early-phase & emetogenic \\
\hline discrete & dose-ranging & early-stage & emotional \\
\hline discriminant & dose-related & easier & empiric \\
\hline discriminative & dosing & east & empirical \\
\hline discriminatory & double & eastern & empty \\
\hline diseased & double-blind & easy & emptying \\
\hline disease-free & double-blinded & eccentric & enalapril \\
\hline disease-modifying & double-dummy & echocardiographic & enantioselective \\
\hline disease-related & double-masked & ecological & encapsulated \\
\hline disease-specific & doubtful & economic & encouraging \\
\hline disordered & down-regulated & economical & end-diastolic \\
\hline disparate & downward & ed & endemic \\
\hline dispersible & doxorubicin & educational & end-expiratory \\
\hline dispositional & dpp-4 & eeg & endocrine \\
\hline disproportionate & dramatic & efalizumab & end-of-study \\
\hline disruptive & drop-out & effective & endogenous \\
\hline dissatisfied & drug-eluting & effect-site & endometrial \\
\hline disseminated & drug-free & effervescent & endoscopic \\
\hline dissociable & drug-induced & efficacious & endothelial \\
\hline distal & drug-naïve & efficient & endothelium-dependent \\
\hline distant & drug-naive & egfr & endotracheal \\
\hline distilled & drug-related & eicosapentaenoic & endovascular \\
\hline distinct & drug-resistant & eighth & endpoint \\
\hline
\end{tabular}




\begin{tabular}{|c|c|}
\hline end-point & esomeprazole \\
\hline end-stage & esophageal \\
\hline end-systolic & essential \\
\hline end-tidal & established \\
\hline energy-restricted & estimable \\
\hline english & estimated \\
\hline english-speaking & etanercept \\
\hline enhanced & etexilate \\
\hline enjoyable & ethical \\
\hline enlarged & ethinyl \\
\hline enormous & ethnic \\
\hline enough & etoposide \\
\hline enriched & etoricoxib \\
\hline enrolled & euglycaemic \\
\hline enteral & euglycemic \\
\hline enteric & euglycemic- \\
\hline enteric-coated & hyperinsulinemic \\
\hline enterohepatic & european \\
\hline entire & euroqol \\
\hline environmental & euroqol-5d \\
\hline enzymatic & evaluable \\
\hline enzyme & evaluated \\
\hline enzyme-linked & evaluative \\
\hline eortc & evaluator-blind \\
\hline eosinophil & evaluator-blinded \\
\hline eosinophilic & event \\
\hline epicardial & event-driven \\
\hline epidemic & event-free \\
\hline epidemiological & event-related \\
\hline epidermal & eventual \\
\hline epidural & everolimus \\
\hline epigastric & every-2-week \\
\hline episodic & everyday \\
\hline epithelial & evidence-based \\
\hline eq-5d & evident \\
\hline equal & evoked \\
\hline equianalgesic & evolutionary \\
\hline equipotent & exacerbated \\
\hline equivalent & exact \\
\hline equivocal & exaggerated \\
\hline er & examiner-blind \\
\hline erectile & examiner-blinded \\
\hline ergogenic & excellent \\
\hline ergonomic & excess \\
\hline erlotinib & excessive \\
\hline erosive & excised \\
\hline er-positive & excisional \\
\hline ertapenem & excitatory \\
\hline erythrocyte & exciting \\
\hline erythromycin & exclusive \\
\hline erythromycin-treated & excreted \\
\hline rythropoiesis-stimulating & executive \\
\hline rythropoietic & exenatide \\
\hline
\end{tabular}

exercise-based

exercise-induced

exercise-related

exert

exertional

exhaled

exhibited

existing

exogenous

expanded

expected

expensive

experienced

experimental

expert

expiratory

explanatory

explicit

explorative

exploratory

exponential

exposed

expressive

extended

extensive

external

extra

extracellular

extracorporeal

extrahepatic

extrapyramidal

extravascular

extreme

extrinsic

ezetimibe

face-to-face

facial

facilitated

facility-based

factorial

faecal

failed

fair

fall-related

false-positive

familial

familiar

family-based

family-focused

famotidine

fast

fasted

faster fastest

fasting

fast-track

fat

fatal

fat-free

fat-rich

fatty

favorable

favourable

fda-approved

fearful

feasible

febrile

febuxostat

fecal

fellow

female

feminine

femoral

fenofibrate

fenofibric

fentanyl

fermented

ferric

ferrous

fesoterodine

fetal

fev1

few

fewer

fexofenadine

fibrate

fibre

fibrinolytic

fibroblast

fidaxomicin

fifth

fifty

fifty-eight

filamentous

film-coated

final

financial

finasteride

fine

finnish

firm

first

first-degree

first-dose

first-episode

first-generation 


\begin{tabular}{|c|c|}
\hline first-in-class & fortified \\
\hline first-in-human & forty-eight \\
\hline first-in-man & forward \\
\hline first-line & four-arm \\
\hline first-order & four-day \\
\hline first-pass & fourfold \\
\hline first-phase & four-fold \\
\hline first-time & four-month \\
\hline first-year & four-period \\
\hline five-day & four-point \\
\hline five-period & fourth \\
\hline five-point & four-treatment \\
\hline five-way & four-way \\
\hline five-year & four-week \\
\hline fixed & four-year \\
\hline fixed-dose & foveal \\
\hline flare & fractional \\
\hline flat & fractionated \\
\hline flexible & fracture \\
\hline flexible-dose & fragile \\
\hline flow-mediated & fragmented \\
\hline fluconazole & frail \\
\hline fluid & frailty \\
\hline flu-like & free \\
\hline fluorescent & free-living \\
\hline fluoride & free-radical \\
\hline fluoroscopic & french \\
\hline fluoxetine & frequent \\
\hline fluoxetine-treated & fresh \\
\hline flush & frontal \\
\hline flushing & front-line \\
\hline fmri & frozen \\
\hline focal & full \\
\hline folate & full-scale \\
\hline folfiri & full-term \\
\hline folic & full-time \\
\hline folinic & fulvestrant \\
\hline follicle-stimulating & fumarate \\
\hline follicular & functional \\
\hline followed-up & fundamental \\
\hline following & fundus \\
\hline followup & fungal \\
\hline follow-up & furoate \\
\hline food-effect & furosemide \\
\hline forced & further \\
\hline force-titrated & future \\
\hline forearm & $\mathrm{g}$ \\
\hline foreign & gag-specific \\
\hline formal & gamma-aminobutyric \\
\hline formative & gaseous \\
\hline former & gastric \\
\hline formoterol & gastroduodenal \\
\hline formula-fed & gastroesophageal \\
\hline
\end{tabular}

\begin{tabular}{|c|c|}
\hline gastrointestinal & glucose-related \\
\hline gastro-intestinal & glucose-stimulated \\
\hline gastrointestinal-related & glutamate \\
\hline gastro-oesophageal & glutamatergic \\
\hline gefitinib & gluteal \\
\hline gel & glycaemic \\
\hline gelatinase-associated & glycated \\
\hline gel-forming & glycemic \\
\hline genant-modified & glycosylated \\
\hline gender-matched & goal-directed \\
\hline general & gold \\
\hline generalisable & golimumab \\
\hline generalised & good \\
\hline generalizable & go-raise \\
\hline generalized & gout \\
\hline gene-related & g-protein-coupled \\
\hline generic & graded \\
\hline genetic & gradual \\
\hline genital & gram-negative \\
\hline genome & gram-positive \\
\hline genome-wide & granulocyte \\
\hline genomic & graphic \\
\hline genotype & graphical \\
\hline genotyped & gray \\
\hline genotypic & great \\
\hline gentamicin & greater \\
\hline gentle & greatest \\
\hline genuine & green \\
\hline geographic & grey \\
\hline geographical & gross \\
\hline geometric & group-based \\
\hline geriatric & guideline-based \\
\hline german & gynaecological \\
\hline gestational & gynecological \\
\hline gh & $\mathrm{h}$ \\
\hline gh-deficient & h1n1 \\
\hline gh-releasing & h2-receptor \\
\hline gh-treated & h5n1 \\
\hline gi & habitual \\
\hline glargine & haematological \\
\hline glaxosmithkline & haemodynamic \\
\hline global & haemoglobin \\
\hline glomerular & haemophilus \\
\hline glp-1 & haemostatic \\
\hline glucagon-like & half \\
\hline glucocorticoid & half-life \\
\hline glucocorticoid-induced & haloperidol \\
\hline glucodynamic & haloperidol-controlled \\
\hline glucoregulatory & haloperidol-treated \\
\hline glucose & ham-d-17 \\
\hline glucose-6-phosphate & hand-foot \\
\hline glucose-dependent & hand-held \\
\hline glucose-lowering & hands-on \\
\hline
\end{tabular}




\begin{tabular}{|c|c|c|c|}
\hline happy & high-calorie & hospitalized & hypoxic \\
\hline haptic & high-carbohydrate & hot & iatrogenic \\
\hline haq-di & high-density & hourly & ibandronate \\
\hline hard & high-dose & hp & ibuprofen \\
\hline harmful & high-energy & hpv & $\mathrm{icu}$ \\
\hline harmonic & higher & $\mathrm{hr}$ & ideal \\
\hline hazard & higher-dose & hrqol & identical \\
\hline hazardous & higher-order & hscrp & idiopathic \\
\hline $\mathrm{hb}$ & highest & hs-crp & ifn $\tilde{A} \ddot{Y}-1 b$ \\
\hline hbalc & high-fat & hsv-2 & ige-mediated \\
\hline hbeag-positive & high-gi & http & igfbp-3 \\
\hline hcg & high-grade & human & igf-i \\
\hline hcl & high-intensity & human-bovine & i-ii \\
\hline hcv-infected & high-level & humanized & il-1 \\
\hline hdl-c & high-performance & humoral & il-10 \\
\hline head-to-head & high-pressure & hyaluronic & il-1 $\tilde{A} \ddot{Y}$ \\
\hline healing & high-protein & hybrid & il-1beta \\
\hline healthcare & high-quality & hydrated & il-2 \\
\hline health-care & high-resolution & hydrochloride & ileal \\
\hline healthful & high-risk & hydrochlorothiazide & iliac \\
\hline healthier & high-sensitivity & hydrocodone & ill \\
\hline health-promoting & high-throughput & hydrolyzed & illicit \\
\hline health-related & hippocampal & hydrophilic & illusory \\
\hline healthy & hispanic & hydroxy & iloperidone \\
\hline heartburn-free & histological & hydroxyapatite & imiquimod \\
\hline heat-sensitive & histomorphometric & hydroxyapatite-coated & immature \\
\hline heavy & histopathological & hydroxychloroquine & immediate \\
\hline hedonic & historic & hydroxynefazodone & immediate-release \\
\hline heightened & historical & hyperbaric & immigrant \\
\hline helminth & hiv-1 & hypercholesterolaemic & immune \\
\hline helpful & hiv-1-infected & hypercholesterolemic & immune-mediated \\
\hline hematological & hiv-infected & hyperglycaemic & immune-related \\
\hline hematopoietic & hiv-negative & hyperglycemic & immunized \\
\hline hemodynamic & hiv-positive & hyperinsulinaemic & immunocompetent \\
\hline hemorrhagic & hiv-related & hyperinsulinemic & immunocompromised \\
\hline hemostatic & hiv-seronegative & hyperinsulinemic- & immunogenic \\
\hline hepatic & hiv-specific & euglycemic & immunohistochemical \\
\hline hepatocellular & hmg-coa & hyperlipidemic & immunologic \\
\hline heptavalent & hoc & hypertensive & immunological \\
\hline her2-negative & holistic & hypertonic & immunomodulatory \\
\hline her2-positive & home-based & hypertrophic & immunoreactive \\
\hline herbal & homeopathic & hypnotic & immunosorbent \\
\hline hereditary & homeostatic & hypoactive & immunostimulatory \\
\hline heterogeneous & homogeneous & hypocaloric & immunosuppressant \\
\hline heterologous & homogenous & hypoglycaemic & immunosuppressive \\
\hline heterosexual & homologous & hypoglycemic & immunotherapy \\
\hline heterozygous & homozygous & hypogonadal & impacted \\
\hline hexavalent & horizontal & hypotensive & impaired \\
\hline hg & hormonal & hypothalamic & impeding \\
\hline hidden & hormone-receptor-positive & hypothalamic-pituitary- & imperative \\
\hline hierarchical & hormone-sensitive & adrenal & implant \\
\hline high & hospital-acquired & hypothesized & implantable \\
\hline high-calcium & hospital-based & hypothetical & implanted \\
\hline
\end{tabular}




\begin{tabular}{|c|c|c|c|}
\hline implementation & ineffective & innate & interdisciplinary \\
\hline implicit & inefficient & inner & interested \\
\hline important & ineligible & inner-city & interesting \\
\hline impossible & inert & innovative & interferon \\
\hline improved & inexpensive & inoperable & interferon-inducible \\
\hline improving & inexperienced & inotropic & interim \\
\hline impulsive & infant & inpatient & interindividual \\
\hline inaccurate & infantile & in-patient & inter-individual \\
\hline inactivated & infected & in-person & interleukin \\
\hline inactive & infection-related & insecticide-treated & interleukin-1 \\
\hline inadequate & infectious & insensitive & interleukin-10 \\
\hline inadvertent & infective & insignificant & interleukin-1 $\tilde{\mathrm{A}} \ddot{\mathrm{Y}}$ \\
\hline inappropriate & inferior & insoluble & interleukin-2 \\
\hline inattentive & infertile & inspiratory & intermediate \\
\hline incisional & infinite & inspired & intermediate-acting \\
\hline inclusive & inflamed & instantaneous & intermittent \\
\hline incompatible & inflammatory & in-stent & internal \\
\hline incomplete & infliximab & institutional & international \\
\hline inconclusive & infliximab-treated & instructional & internet \\
\hline incongruent & influential & instructive & internet-based \\
\hline inconsistent & influenza & instrumental & internet-delivered \\
\hline incontinent & influenzae & instrumented & interpatient \\
\hline incorporate & influenza-like & insufficient & inter-patient \\
\hline incorporated & informal & insulin-dependent & interpersonal \\
\hline incorporating & informational & insulinemic & interpretable \\
\hline incorrect & informative & insulin-independent & interproximal \\
\hline increased & informed & insulin-induced & interquartile \\
\hline incremental & infrared & insulin-like & interrupted \\
\hline incurable & infrequent & insulin-mediated & interstitial \\
\hline indacaterol & infused & insulin-naïve & intersubject \\
\hline independent & infusional & insulin-naive & inter-subject \\
\hline in-depth & infusion-related & insulinogenic & interventional \\
\hline indexed & ingested & insulin-resistant & interview-based \\
\hline indian & inguinal & insulin-sensitizing & intestinal \\
\hline indicated & inhalational & insulin-stimulated & intestine \\
\hline indicative & inhaled & insulin-treated & intimal \\
\hline indigenous & inhaler & intact & intimate \\
\hline indirect & inherent & intake & intolerable \\
\hline indistinguishable & inhibited & integral & intolerant \\
\hline individual & inhibiting & integrated & intra \\
\hline individualised & inhibitor & integrative & intraabdominal \\
\hline individualized & inhibitor-1 & intellectual & intra-abdominal \\
\hline individual-level & inhibitor-based & intended & intra-arterial \\
\hline individually-tailored & inhibitory & intense & intraarticular \\
\hline indocyanine & in-home & intensive & intra-articular \\
\hline indole & in-hospital & intentional & intrabony \\
\hline indomethacin & initial & intention-to-treat & intracellular \\
\hline indoor & initiating & intent-to-treat & intracerebral \\
\hline induced & injectable & interactive & intracoronary \\
\hline inducible & injected & intercellular & intracortical \\
\hline inductive & injection-site & interchangeable & intracranial \\
\hline industrial & injured & intercompartmental & intractable \\
\hline industrialized & injurious & intercostal & intracytoplasmic \\
\hline
\end{tabular}




\begin{tabular}{|c|c|c|c|}
\hline intradermal & irinotecan & laryngeal & likert \\
\hline intraepithelial & irregular & laryngoscopy & limbic \\
\hline intragastric & irrelevant & last & limited \\
\hline intrahepatic & irrespective & lasting & linear \\
\hline intraindividual & irreversible & last-observation-carried- & linezolid \\
\hline intra-individual & irritable & forward & lingual \\
\hline intramuscular & irritant & latanoprost & linguistic \\
\hline intramyocellular & ischaemic & late & linoleic \\
\hline intranasal & ischemic & late-life & lipid \\
\hline intraocular & isocaloric & latency & lipid-altering \\
\hline intraoperative & isoenergetic & latent & lipid-lowering \\
\hline intra-operative & isokinetic & late-onset & lipid-modifying \\
\hline intraoral & isolated & late-phase & lipophilic \\
\hline intraperitoneal & isometric & later & liposomal \\
\hline ntrapulmonary & isosorbide-5-mononitrate & lateral & liquid \\
\hline intrasubject & isotonic & latest & lispro \\
\hline intra-subject & isrctn & late-stage & little \\
\hline intrathecal & italian & latin & live \\
\hline intratumoral & iterative & latin-square & live-attenuated \\
\hline intrauterine & iu & latter & living \\
\hline intravaginal & japanese & lavage & local \\
\hline intravascular & joint & laxative & localised \\
\hline intravenous & jugular & ldl & localized \\
\hline ntraventricular & juvenile & ldl-c & locoregional \\
\hline ntravitreal & kaplan-meier & ldl-cholesterol & logarithmic \\
\hline in-treatment & kcal & ldl-c-lowering & logical \\
\hline intriguing & ketoconazole & le & logistic \\
\hline intrinsic & key & lead & logistical \\
\hline intrusive & $\mathrm{kg}$ & lead-in & log-linear \\
\hline intubated & kg-1 & lean & log-rank \\
\hline intuitive & kinematic & learned & log-transformed \\
\hline invariant & kinetic & least & long \\
\hline invasive & known & least-square & long-acting \\
\hline inverse & korean & led & long-chain \\
\hline inverted & 1 & left & longer \\
\hline investigational & 11 & left-sided & longer-term \\
\hline nvestigative & labelled & legal & longest \\
\hline nvestigator-administered & laboratory-based & leisure-time & longitudinal \\
\hline nvestigator-blind & laboratory-confirmed & lengthy & long-lasting \\
\hline investigator-blinded & lacosamide & leptin & long-standing \\
\hline nvestigator-initiated & lactic & lersivirine & longterm \\
\hline investigator-rated & lactobacilli & lesional & long-term \\
\hline in-vitro & lactobacillus & less & loose \\
\hline in-vivo & lai & lesser & loperamide \\
\hline involuntary & lamivudine & lethal & lopinavir \\
\hline involved & lamotrigine & liberal & loracarbef \\
\hline iodine & lansoprazole & licensed & loracarbef-treated \\
\hline iohexol & laparoscopic & lifelong & loratadine \\
\hline iontophoretic & large & lifestyle & lot-to-lot \\
\hline iop-lowering & larger & life-threatening & lovastatin \\
\hline pilimumab & large-scale & light & low \\
\hline psilateral & largest & lighter & low-active \\
\hline canian & laropiprant & likely & low-back \\
\hline
\end{tabular}




\begin{tabular}{|c|c|c|c|}
\hline low-calorie & manual & methicillin-resistant & $\mathrm{ml}$ \\
\hline low-carbohydrate & manualized & methicillin-susceptible & $\mathrm{ml}-1$ \\
\hline low-cost & many & methodological & $\mathrm{mm}$ \\
\hline low-density & marginal & methotrexate & mmhg \\
\hline low-dose & marital & methotrexate-naive & mmol \\
\hline lower & marked & metoprolol & mmp-3 \\
\hline lower-dose & masked & metric & mobile \\
\hline lowest & mass & metronidazole & mobile-bearing \\
\hline low-fat & matching & metropolitan & mobility \\
\hline low-gi & maternal & mexican & mock \\
\hline low-glycemic & mathematical & mf59-adjuvanted & modafinil \\
\hline low-grade & mature & $\mathrm{mg}$ & modal \\
\hline low-income & maximal & micro & model-based \\
\hline low-intensity & maximum & microbial & modeled \\
\hline low-level & maximum-tolerated & microbiologic & modeling \\
\hline low-molecular-weight & $\operatorname{mcg}$ & microbiological & modelled \\
\hline low-risk & mcrc & microcirculatory & modelling \\
\hline low-trauma & meal-induced & microg & moderate \\
\hline lumbar & meal-related & micronized & moderated \\
\hline luminal & mealtime & micronutrient & moderate-dose \\
\hline lumiracoxib & mean & microscopic & moderate-fat \\
\hline lupus & meaningful & microsomal & moderate-intensity \\
\hline luteal & measurable & microvascular & moderate-to-severe \\
\hline lymph & measured & mid & moderate-to-vigorous \\
\hline lymphatic & mechanical & middle & moderating \\
\hline lymphoblastic & mechanism-based & middle-aged & modern \\
\hline lymphocyte & mechanistic & migraine & modest \\
\hline lymphoproliferative & medial & migraine-associated & modifiable \\
\hline macronutrient & median & migraine-related & modified \\
\hline macrovascular & mediastinal & migraine-specific & modular \\
\hline macular & mediate & mild & modulate \\
\hline magnetic & mediated & milder & modulated \\
\hline main & mediating & mild-moderate & modulating \\
\hline major & medical & mild-to-moderate & modulatory \\
\hline maladaptive & medication-free & military & moisturizing \\
\hline malaria-endemic & medicinal & milk-based & molar \\
\hline malarial & medium & mimetic & molecular \\
\hline malaria-naïve & medium-term & mindfulness-based & monetary \\
\hline male & mefloquine & minimal & monitored \\
\hline maleate & mellitus & mini-mental & monocentric \\
\hline malignant & memantine & minimum & monoclonal \\
\hline malnourished & meningococcal & minor & monocular \\
\hline mammalian & menopausal & minute & monogamous \\
\hline mammographic & menstrual & mismatched & mononuclear \\
\hline manageable & mental & missing & monophasic \\
\hline mandatory & metabolic & mitochondrial & monotherapy \\
\hline mandible & metabolite & mitogen-activated & monounsaturated \\
\hline mandibular & metallic & mitral & monovalent \\
\hline manic & metastatic & mitt & monoxide-confirmed \\
\hline manifest & metered & mixed & montelukast \\
\hline manipulative & metered-dose & mixed-effect & monthly \\
\hline mantel-haenszel & metformin & mixed-effects & moral \\
\hline mantle & metformin-treated & mixed-model & more \\
\hline
\end{tabular}




\begin{tabular}{|c|c|c|c|}
\hline moreover & multisite & negligible & night-time \\
\hline morphine-induced & multi-site & nelfinavir & nilotinib \\
\hline morphological & multi-stage & neoadjuvant & ninety-eight \\
\hline morphometric & multitargeted & neointimal & ninth \\
\hline most & multivalent & neonatal & nitrate \\
\hline motivated & multivariable & neoral & nitric \\
\hline motivational & multivariable-adjusted & neovascular & nitrous \\
\hline moxifloxacin & multivariate & nephrotic & nizatidine \\
\hline $\operatorname{mrcc}$ & multivariate-adjusted & nervous & $\mathrm{nm}$ \\
\hline mri & municipal & net & nnrti-resistant \\
\hline mrna & mu-opioid & neural & nociceptive \\
\hline $\mathrm{mtx}$ & muscular & neuraminidase & nocturnal \\
\hline much & musculoskeletal & neurobehavioral & nodal \\
\hline mucous & musical & neurobiological & node-positive \\
\hline mug & mutant & neurochemical & no-effect \\
\hline multi & mutated & neurocognitive & nominal \\
\hline multicenter & mutational & neurodevelopmental & non \\
\hline multi-center & mutual & neuroendocrine & nonadherent \\
\hline multicentered & mycobacterial & neurogenic & nonadjuvanted \\
\hline multicentre & mycophenolate & neurohormonal & non-adjuvanted \\
\hline multi-centre & mycophenolic & neuroimaging & nonalcoholic \\
\hline multicentric & myelodysplastic & neuroleptic & non-alcoholic \\
\hline multicompartmental & myelogenous & neurologic & non-blind \\
\hline multicomponent & myeloid & neurological & nonblinded \\
\hline multi-component & myocardial & neuromuscular & non-blinded \\
\hline multidimensional & $n-3$ & neuronal & noncancer \\
\hline multidisciplinary & naïve & neuropathic & noncardiac \\
\hline multi-disciplinary & naive & neurophysiological & non-clinical \\
\hline multidose & naproxen & neuroprotective & non-communicable \\
\hline multi-dose & narrative & neuropsychiatric & noncomparative \\
\hline multidrug & narrow & neuropsychological & noncompartmental \\
\hline multidrug-resistant & nasal & neurosensory & non-compartmental \\
\hline multi-ethnic & nasogastric & neurosurgical & non-coronary \\
\hline multifaceted & nasopharyngeal & neurotrophic & nondepressed \\
\hline multi-faceted & nateglinide & neurovascular & nondiabetic \\
\hline multifactorial & national & neutral & non-diabetic \\
\hline multi-factorial & nationwide & neutralizing & non-disabled \\
\hline multifocal & native & neutropenic & non-dominant \\
\hline multifunctional & natriuretic & neutrophil & non-elderly \\
\hline multi-institutional & natural & new & non-exercise \\
\hline multi-item & naturalistic & newborn & nonfasting \\
\hline multikinase & nausea & newer & nonfatal \\
\hline multilevel & n-back & new-onset & non-fatal \\
\hline multi-level & near & next & non-hdl \\
\hline multimodal & nearest & next-day & non-hdl-c \\
\hline multinational & near-infrared & next-generation & non-hematological \\
\hline multinomial & near-maximal & ng & non-high-density \\
\hline multiple & nebulized & niacin & non-hispanic \\
\hline multiple-ascending & necessary & niacin-induced & non-hodgkin \\
\hline multiple-choice & necrotic & nicotine & nonhuman \\
\hline multiple-dose & nefazodone & nicotinic & noninfectious \\
\hline multiplex & negative & nifedipine & noninferior \\
\hline multi-professional & negatively & nighttime & non-inferior \\
\hline
\end{tabular}




\begin{tabular}{|c|c|c|c|}
\hline non-inferiority & non-small-cell & nulliparous & once-daily \\
\hline non-inflammatory & nonsmoking & numeric & once-monthly \\
\hline non-insulin & non-smoking & numerical & once-weekly \\
\hline non-insulin-dependent & nonspecific & numerous & once-yearly \\
\hline non-intervention & non-specific & nurse-delivered & oncogenic \\
\hline noninvasive & nonsquamous & nurse-led & on-demand \\
\hline non-invasive & non-squamous & nutrient & one-compartment \\
\hline non-japanese & nonsteroidal & nutritional & one-fifth \\
\hline nonlinear & non-steroidal & obese & one-half \\
\hline non-linear & nonstructural & obesity-related & one-hour \\
\hline nonlipid & non-st-segment & objective & one-month \\
\hline non-malignant & non-supervised & objectively & one-sided \\
\hline non-medical & nonsurgical & observable & one-tailed \\
\hline nonmetastatic & non-surgical & observational & one-third \\
\hline non-motor & non-tailored & observed & one-time \\
\hline nonmyeloid & non-traumatic & observer-blind & one-to-one \\
\hline non-neuropathic & non-treatment & observer-blinded & one-way \\
\hline nonnucleoside & nontypeable & observer-masked & one-week \\
\hline non-nucleoside & non-typeable & obsessive-compulsive & one-year \\
\hline nonobese & non-vaccine & obstetric & ongoing \\
\hline non-obese & non-verbal & obstetrical & on-going \\
\hline nonocular & nonvertebral & obstructive & online \\
\hline non-opioid & noradrenergic & obtainable & on-line \\
\hline non-palpable & nordic & obvious & only \\
\hline nonparametric & norepinephrine & oc & onset \\
\hline non-parametric & norethindrone & occasional & on-site \\
\hline nonpeptide & norethisterone & occipital & on-study \\
\hline non-peptide & norfloxacin & occlusal & on-treatment \\
\hline non-pharmaceutical & norgestimate & occlusive & oocyte \\
\hline nonpharmacological & normal & occult & opaque \\
\hline non-pharmacological & normalised & occupational & open \\
\hline non-platinum & normalized & ocular & open-angle \\
\hline nonpregnant & normal-weight & odd & open-ended \\
\hline non-pregnant & normative & oesophageal & open-label \\
\hline non-prescription & normotensive & oestradiol & open-labeled \\
\hline nonprogressive & north & off-drug & operable \\
\hline non-progressive & northern & office-based & operational \\
\hline nonpsychotic & norwegian & off-label & operative \\
\hline non-randomised & nosocomial & off-pump & ophthalmic \\
\hline nonrandomized & notable & olanzapine & opiate \\
\hline non-randomized & noteworthy & olanzapine-fluoxetine & opioid \\
\hline nonrenal & noticeable & olanzapine-treated & opioid-induced \\
\hline non-responder & no-treatment & old & opioid-related \\
\hline non-response & novel & older & opportunistic \\
\hline nonresponsive & noxious & oldest & opposite \\
\hline nonselective & $\mathrm{nph}$ & oleic & oppositional \\
\hline non-selective & n-telopeptide & oligomeric & opsonophagocytic \\
\hline nonserious & n-terminal & oligonucleotide & optic \\
\hline non-serious & nuclear & olive & optical \\
\hline non-severe & nucleoside & omalizumab-treated & optimal \\
\hline nonsignificant & nucleotide & omega-3 & optimised \\
\hline non-significant & nucleus & omeprazole & optimized \\
\hline non-small & null & once-a-day & optimum \\
\hline
\end{tabular}




\begin{tabular}{|c|c|c|c|}
\hline optional & p450-mediated & patient-reported & p-glycoprotein \\
\hline or & paediatric & patient-specific & p-gp \\
\hline oral & pai-1 & pcr-corrected & $\mathrm{ph}$ \\
\hline oral-dose & pain-free & peak & pharmaceutical \\
\hline orange & painful & peaked & pharmacist-led \\
\hline orbitofrontal & painless & peak-to-trough & pharmacodynamic \\
\hline ordinal & pain-related & peak-trough & pharmacoeconomic \\
\hline ordinary & paired & pedal & pharmacogenetic \\
\hline organic & pakistani & pediatric & pharmacogenomic \\
\hline organisational & palatable & pedometer-based & pharmacokinetic \\
\hline organizational & paliperidone & peer & pharmacokinetic- \\
\hline original & palliative & peer-led & pharmacodynamic \\
\hline oropharyngeal & pancreatic & pegfilgrastim & pharmacologic \\
\hline orthopaedic & pandemic & peg-ifn & pharmacological \\
\hline orthopedic & paper-based & pegylated & pharmacoscintigraphic \\
\hline orthostatic & paradoxical & pelvic & pharyngeal \\
\hline oscillatory & parallel & pemetrexed & phase \\
\hline oseltamivir & parallel-arm & penetrating & phase-ii \\
\hline osmotic & parallel-group & pentavalent & phasic \\
\hline osseous & parametric & peptic & ph-dependent \\
\hline osteoblast & paramount & perceptible & phenotypic \\
\hline osteoclast & parasite & perceptual & phid-cv \\
\hline osteopenic & parasitological & percutaneous & phosphate \\
\hline osteoporotic & parasympathetic & perennial & phospholipid \\
\hline otamixaban & parent & perfect & phosphorylated \\
\hline other & parental & performance-based & photodamaged \\
\hline outcome & parent-child & perinatal & photodynamic \\
\hline outdoor & parenteral & perineal & photographic \\
\hline outer & parent-focused & periodic & phq-9 \\
\hline out-of-pocket & parent-reported & periodontal & physical \\
\hline outpatient & paretic & perioperative & physician \\
\hline out-patient & paroxetine & peri-operative & physician-assessed \\
\hline outreach & paroxetine-controlled & peripheral & physician-patient \\
\hline outside & paroxysmal & periprocedural & physicochemical \\
\hline ovarian & partial & periprosthetic & physiologic \\
\hline ovary & particular & peritoneal & physiological \\
\hline overactive & particulate & peri-urban & physiotherapy \\
\hline overall & pasi & permanent & pi-based \\
\hline overlap & passive & permuted & pilot \\
\hline overlapping & past & peroral & pituitary \\
\hline overnight & patellofemoral & peroxisome & pivotal \\
\hline overt & pathogenic & per-patient & $\mathrm{pk}$ \\
\hline over-the-counter & pathological & per-protocol & placebo \\
\hline overweight & pathophysiological & persistent & placebo-adjusted \\
\hline ovulatory & patient & personal & placebo-controlled \\
\hline own & patient-blinded & personalised & placebo-corrected \\
\hline oxaliplatin & patient-centered & personalized & placebo-subtracted \\
\hline oxaliplatin-based & patient-centred & persuasive & placebo-treated \\
\hline oxidant & patient-controlled & pertinent & placental \\
\hline oxidative & patient-level & pertussis-inactivated & plain \\
\hline oxidized & patient-perceived & pervasive & planned \\
\hline oxygen-derived & patient-rated & pg & plaque \\
\hline $\mathrm{p}$ & patient-related & pgi-i & platelet-rich \\
\hline
\end{tabular}




\begin{tabular}{|c|c|c|c|}
\hline platinum-based & postoperative & pre-existing & preventing \\
\hline platinum-sensitive & post-operative & preferable & preventive \\
\hline plausible & postoperatively & preference-based & previous \\
\hline pleasant & post-operatively & preferential & primaquine \\
\hline pleiotropic & postpartum & preferred & primary \\
\hline pleural & postprandial & prefrontal & prime \\
\hline pneumatic & post-prandial & pregabalin-treated & prime-boost \\
\hline pneumococcal & post-primary & pregnant & primed \\
\hline point-of-care & postprocedural & prehospital & priming \\
\hline poliovirus & post-stroke & prehypertensive & primiparous \\
\hline polyclonal & post-study & pre-intervention & principal \\
\hline polycystic & postsurgical & preliminary & print-based \\
\hline polymeric & posttherapy & premalignant & prior \\
\hline polymorphic & post-therapy & premature & priori \\
\hline polysomnographic & post-training & pre-meal & private \\
\hline polyunsaturated & post-transplant & premenopausal & proactive \\
\hline pooled & posttraumatic & premenstrual & probabilistic \\
\hline poor & post-traumatic & premier & probable \\
\hline poorer & post-treatment & premixed & probing \\
\hline popular & postural & prenatal & probiotic \\
\hline population-based & postvoid & preoperative & problematic \\
\hline population-level & potent & pre-operative & problem-solving \\
\hline population-specific & potential & prepandemic & pro-brain \\
\hline porous & powered & preparatory & procedural \\
\hline portable & powerful & prepared & procedure-related \\
\hline portal & ppm & preplanned & productive \\
\hline posaconazole & practical & pre-planned & professional \\
\hline positive & practice-based & pre-post & profibrinolytic \\
\hline positive-controlled & pragmatic & preprandial & proficient \\
\hline possible & pramlintide & pre-pregnancy & profile \\
\hline postabsorptive & pramlintide-treated & prepubertal & profound \\
\hline post-acute & prandial & pre-school & prognostic \\
\hline postal & pravastatin & prescribed & progression-free \\
\hline post-baseline & pre & present & progressive \\
\hline post-brushing & prebiotic & preservative-free & proinflammatory \\
\hline post-challenge & pre-brushing & preserved & pro-inflammatory \\
\hline post-discharge & precancerous & prespecified & prolactin-related \\
\hline postdose & precise & pre-specified & proliferative \\
\hline post-dose & preclinical & pressurised & proliferator-activated \\
\hline post-dosing & pre-clinical & pressurized & prolong \\
\hline posterior & predefined & pre-study & prolonged \\
\hline posterolateral & pre-defined & presumed & prominent \\
\hline post-exercise & predetermined & presumptive & promising \\
\hline post-exposure & pre-determined & presystemic & prompt \\
\hline postherpetic & predictable & preterm & prone \\
\hline posthoc & predictive & pre-test & pronounced \\
\hline post-hoc & prednisolone & pretreated & propellant-free \\
\hline post-intervention & predominant & pretreatment & proper \\
\hline postmenopausal & pre-dose & pre-treatment & prophylactic \\
\hline post-menopausal & preemptive & pre-vaccination & propionate \\
\hline postmenstrual & pre-emptive & prevalent & propofol \\
\hline postnatal & pre-erythrocytic & preventable & proportional \\
\hline post-natal & pre-exercise & preventative & proposed \\
\hline
\end{tabular}




\begin{tabular}{|c|c|c|c|}
\hline proprietary & qualified & ready & renal \\
\hline proprioceptive & qualifying & real & renoprotective \\
\hline prosocial & qualitative & realistic & repeatable \\
\hline prospective & quality-adjusted & real-life & repeat-dose \\
\hline prospectively & quality-of-life & real-time & repeated \\
\hline prostate-specific & quantifiable & reasonable & repeated-dose \\
\hline prostatic & quantitative & reassortant & repetitive \\
\hline prosthetic & quarterly & receiver-operating & replicate \\
\hline protease-activated & quartile & recent & reported \\
\hline proteasome & quasi-experimental & recent-onset & representative \\
\hline protective & questionable & receptive & reproducible \\
\hline prothrombin & quetiapine & receptor-1 & reproductive \\
\hline protocol-defined & quick & receptor-mediated & required \\
\hline protocol-specified & quicker & receptor-positive & resectable \\
\hline prototypical & quiet & reciprocal & resected \\
\hline proven & $\mathrm{r}$ & recognised & resident \\
\hline provincial & rabeprazole & recombinant & residential \\
\hline provisional & racemic & recommended & residual \\
\hline provocative & racial & recorded & resistance-associated \\
\hline proximal & radial & recreational & resistant \\
\hline proxy & radical & rectal & resistive \\
\hline psoriatic & radicular & recurrence-free & resource-limited \\
\hline psychiatric & radioactive & recurrent & respective \\
\hline psychic & radiographic & red & respiratory \\
\hline psychodynamic & radiolabeled & reduced & responsible \\
\hline psychoeducational & radiolabelled & redundant & responsive \\
\hline psycho-educational & radiologic & referral & restless \\
\hline psychological & radiological & refined & restorative \\
\hline psychometric & radiostereometric & reflective & restricted \\
\hline psychophysical & radiotherapy & refractive & restrictive \\
\hline psychophysiological & ramelteon & refractory & resultant \\
\hline psychosocial & ramipril & regional & retinal \\
\hline psycho-social & random & registered & retrieval \\
\hline psychotherapeutic & randomised & regular & retrospective \\
\hline psychotic & randomised-controlled & regulating & reverse \\
\hline psychotropic & randomized & regulatory & reversible \\
\hline pubertal & randomized-controlled & rehabilitative & revised \\
\hline public & randomly & relapse & rewarding \\
\hline published & ranibizumab & relapsed & reward-related \\
\hline pulmonary & ranibizumab-treated & relapse-free & rheumatic \\
\hline pulsed & ranitidine & relapsing-remitting & rheumatoid \\
\hline pure & ranitidine-treated & related & ribosomal \\
\hline purpose & rank & relational & rich \\
\hline purulent & rapamycin & relative & rifampicin \\
\hline putative & rapid & relevant & right \\
\hline p-value & rapid-acting & reliable & right-handed \\
\hline pylori-negative & rare & reliever & rigid \\
\hline qd & rash & relieving & rigorous \\
\hline qol & rational & reluctant & risedronate \\
\hline qt & rationale & remarkable & rising-dose \\
\hline qtc & raw & remifentanil & risk-benefit \\
\hline q-twist & reactive & remifentanil-based & risky \\
\hline quadrivalent & reactogenic & remote & risperidone \\
\hline
\end{tabular}




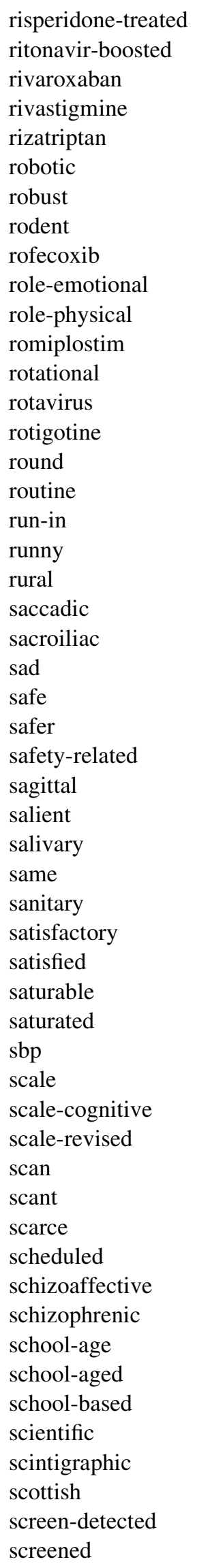

sd

se

seasonal

second

secondary

second-generation

second-line

second-phase

second-year

secretory

sectional

secure

sedated

sedating

sedative

sedentary

segmental

segmented

seizure-free

select

selected

selective

self-administered

self-assessed

self-care

self-completed

self-directed

self-efficacy

self-esteem

self-identified

self-limited

self-limiting

self-management

self-monitored

self-monitoring

self-paced

self-perceived

self-rated

self-report

self-reported

self-selected

semantic

semi-automated

seminal

semiquantitative

semi-quantitative

semistructured

semi-structured

senior

sensitive

sensorineural

sensory

separate sepsis-induced

septal

septic

sequential

sequestered

serial

serious

serological

seronegative

seropositive

seroprotective

serotonergic

serotype-specific

serous

serum

sevelamer

seven-day

seven-point

seventh

seventy

seventy-eight

seventy-five

seventy-one

seventy-two

several

severe

sevoflurane

sex-matched

sex-related

sex-specific

sexual

sf-36

sf-6d

sgrq

sham-controlled

sham-treated

shared

sharp

short

short-acting

short-course

shortened

shorter

short-form

short-lived

short-term

sick

sickle

sick-leave

sick-listed

sickness

side

side-effect sigmoid

sigmoidal

signal-regulated

signed-rank

significant

sildenafil

silent

similar

simple

simpler

simplex

simplified

simulated

simultaneous

simvastatin

single

single-agent

single-arm

single-ascending

single-blind

single-blinded

single-center

single-centre

single-day

single-dose

single-item

single-level

single-masked

single-nucleotide

single-pill

single-rooted

single-site

singleton

singular

sinus

sinusoidal

sirolimus-eluting

site-specific

six-dose

six-minute

six-month

sixth

sixty-eight

sixty-one

six-way

six-week

sizeable

skeletal

skeletal-related

skilled

sle

sleep-related

slight 
slow

slower

small

small-cell

smaller

smallest

small-molecule

smear-positive

smoking-related

smooth

soc

so-called

social

social-cognitive

societal

sociodemographic

socio-demographic

socioeconomic

socio-economic

sodium

sodium-glucose

sofa

soft

soft-tissue

sole

solicited

solid

solid-phase

soluble

somatic

somatosensory

sonic

sophisticated

sorafenib

sore

sound

south

southern

south-west

soy-based

sp

spanish

spanish-speaking

sparse

spatial

spatiotemporal

special

specialised

specialist

specialized

specific

specified

spectral spectrometric

spherical

spinal

spine

spiritual

spirometric

splanchnic

spleen

split

split-face

split-mouth

split-virion

spontaneous

sporadic

sputum

squamous

square

squat

srl

stable

stain

stand-alone

standard

standard-dose

standardised

standardized

standard-of-care

stannous

staphylococcal

static

statin

statin-induced

statin-treated

stationary

statistic

statistical

stavudine

steady

steady-state

steep

stent

stereoselective

stereotactic

sterile

sternal

steroid

stimulant

stimulating

stimulatory

stinging

stochastic

straight

strain-specific strata

strategic

stratified

streptococcal

stressful

stress-induced

stress-related

striatal

strict

striking

stringent

stromal

strong

stronger

strongest

stroop

structural

structured

studied

study

study-specific

subacromial

subacute

sub-acute

subarachnoid

subchronic

subclinical

sub-clinical

subcutaneous

subepithelial

subfoveal

subject

subjective

subject-reported

subject-specific

sublingual

submaximal

subnormal

suboptimal

sub-optimal

sub-saharan

subscale

subsequent

substantial

substantive

substrate

sub-study

subthalamic

subtherapeutic

subthreshold

sub-threshold

subtle

subtype subunit

suburban

successful

successive

such

sucrose

sudden

sufficient

sugar-sweetened

suggestive

suicidal

suitable

sumatriptan

superficial

superimposable

superimposed

superior

supervised

supine

supplemental

supplementary

supplemented

supportive

suppressed

suppressive

supragingival

supratherapeutic

supraventricular

surfactant

surgical

surprising

surrogate

survival

susceptible

suspected

sustainable

sustained

svcam-1

swedish

sweet

swiss

swollen

symbolic

symmetric

symmetrical

sympathetic

symptom

symptomatic

symptom-based

symptom-free

symptom-related

synaptic

synchronous 


\begin{tabular}{|c|c|c|c|}
\hline syncytial & therapist & tolerable & tricyclic \\
\hline synergistic & therapy-naive & tolerate & triple \\
\hline synovial & thermal & tolerated & triple-blind \\
\hline synthetic & thermogenic & toll-like & triple-therapy \\
\hline systematic & thick & tolterodine & triplicate \\
\hline systemic & thin & tonic & trivalent \\
\hline system-related & thiopental & top & troublesome \\
\hline systolic & third & top-down & truncated \\
\hline $\mathrm{t}$ & third-line & topical & tryptophan \\
\hline t1-weighted & third-party & topiramate & tuberculous \\
\hline $\mathrm{t} 2 \mathrm{dm}$ & thirst & topotecan & tubular \\
\hline tacrolimus & thirty-eight & total & tumour \\
\hline tactile & thirty-one & toxic & turkish \\
\hline tadalafil & thoracic & toxoid & tutorial \\
\hline tailored & thorough & trabecular & twelve-month \\
\hline tailor-made & threatening & tracheal & twenty-eight \\
\hline taiwanese & three-arm & traditional & twice \\
\hline tamoxifen & three-armed & trained & twice-a-day \\
\hline tamsulosin & three-day & training-induced & twice-daily \\
\hline tanzanian & three-dimensional & tramadol & twice-weekly \\
\hline taranabant & three-dose & tranexamic & twin \\
\hline target-controlled & threefold & transarterial & two-arm \\
\hline targeted & three-fold & transcranial & two-armed \\
\hline task-related & three-month & transcriptional & two-center \\
\hline tat & three-period & transcutaneous & two-centre \\
\hline tau & three-treatment & transdermal & two-compartment \\
\hline t-cell & three-way & transepidermal & two-dimensional \\
\hline t-cell-mediated & three-week & transfusional & two-dose \\
\hline technical & three-year & transient & two-factor \\
\hline telephone-based & thrice-daily & transitional & twofold \\
\hline telephone-delivered & thromboembolic & translational & two-fold \\
\hline temporal & thrombolytic & transluminal & two-group \\
\hline temporary & thrombotic & transmitted & two-hour \\
\hline tended & thyroid & transparent & two-month \\
\hline tender & tibial & transporter-mediated & two-part \\
\hline tension-type & tibiofemoral & transrectal & two-period \\
\hline ten-year & tid & transtheoretical & two-phase \\
\hline terbinafine & tidal & transthoracic & two-sample \\
\hline terfenadine & tight & transurethral & two-sequence \\
\hline teriparatide & time-averaged & transvaginal & two-sided \\
\hline terminal & time-dependent & traumatic & two-stage \\
\hline tertiary & time-limited & treatment-associated & two-step \\
\hline test & timely & treatment-as-usual & two-tailed \\
\hline tested & time-matched & treatment-emergent & two-treatment \\
\hline test-of-cure & time-weighted & treatment-experienced & two-way \\
\hline testosterone & timolol & treatment-free & two-week \\
\hline tetanus & tinnitus & treatment-induced & two-year \\
\hline tetravalent & tissue-selective & treatment-naïve & tympanic \\
\hline t-helper & titrated & treatment-naive & type \\
\hline theoretical & t-lymphocyte & treatment-related & type-2 \\
\hline theory-based & $\operatorname{tmax}$ & treatment-resistant & type-specific \\
\hline therapeutic & tocilizumab & tremendous & typhoid \\
\hline therapeutical & tofacitinib & trial & typical \\
\hline
\end{tabular}




\section{u-aqp 2}

ubiquitous

uc

uk

ulcerative

ultimate

ultra

ultra-long-acting

ultrasonic

ultrasound

ultrasound-guided

umbilical

unable

unacceptable

unadjusted

unadjuvanted

unaffected

unaltered

unanswered

unanticipated

unavailable

unaware

unbalanced

unbiased

unblinded

unblinding

unboosted

unbound

uncemented

uncertain

unchanged

uncircumcised

unclear

uncomfortable

uncommon

uncomplicated

unconditional

unconjugated

unconscious

uncontrollable

uncontrolled

uncooperative

uncorrected

under-five

undergone

undergraduate

underlying

under-represented

underserved

understudied

undertreated

underwent

undesirable undesired

undetectable

undetected

undetermined

undiagnosed

undifferentiated

unemployed

uneventful

unexpected

unexplained

unexplored

unfamiliar

unfavorable

unfavourable

unfit

unformed

unfractionated

unhealthy

unhelpful

unidentified

unified

uniform

unilateral

unimanual

uninfected

uninjured

unintended

unintentional

uninterrupted

uninvolved

unipolar

unique

univariate

universal

university-affiliated

university-based

unknown

unlabelled

unlikely

unmet

unnecessary

unpaired

unplanned

unpleasant

unpredictable

unprotected

unrecognized

unrelated

unreliable

unresectable

unresolved

unresponsive

unrestricted unsafe

unsatisfactory

unsaturated

unscheduled

unselected

unsolicited

unspecific

unstable

unstimulated

unstructured

unsuccessful

unsuitable

unsupervised

unsupported

untested

untoward

untrained

untranslated

untreated

unusual

unvaccinated

unwanted

unwilling

upcoming

upper

uptitrated

up-titrated

urate

urate-lowering

urban

urgent

uric

urinary

urine

urodynamic

urological

usable

useful

ustekinumab

ustekinumab-treated

usual

usual-care

uterine

uui

vaccinated

vaccine-homologous

vaccine-induced

vaccine-related

vaccine-type

vagal

vaginal

valid

validated valproate

valproic

valuable

valve

valvular

vancomycin

vandetanib

vardenafil

varenicline

variable

variant

varicella

varicella-zoster

varied

various

varnish

varus

vascular

vasoactive

vasodilating

vasodilator-stimulated

vasodilatory

vasogenic

vasomotor

vasopressor

vast

vehicle-controlled

venlafaxine

venous

ventilator-associated

ventilator-free

ventilatory

ventral

ventricular

ventromedial

verapamil

verbal

verified

versus

vertebral

vertebroplasty

vertical

very

vestibular

viable

video

vietnamese

vigorous

vildagliptin

vildagliptin-treated

viral

virologic

virological 


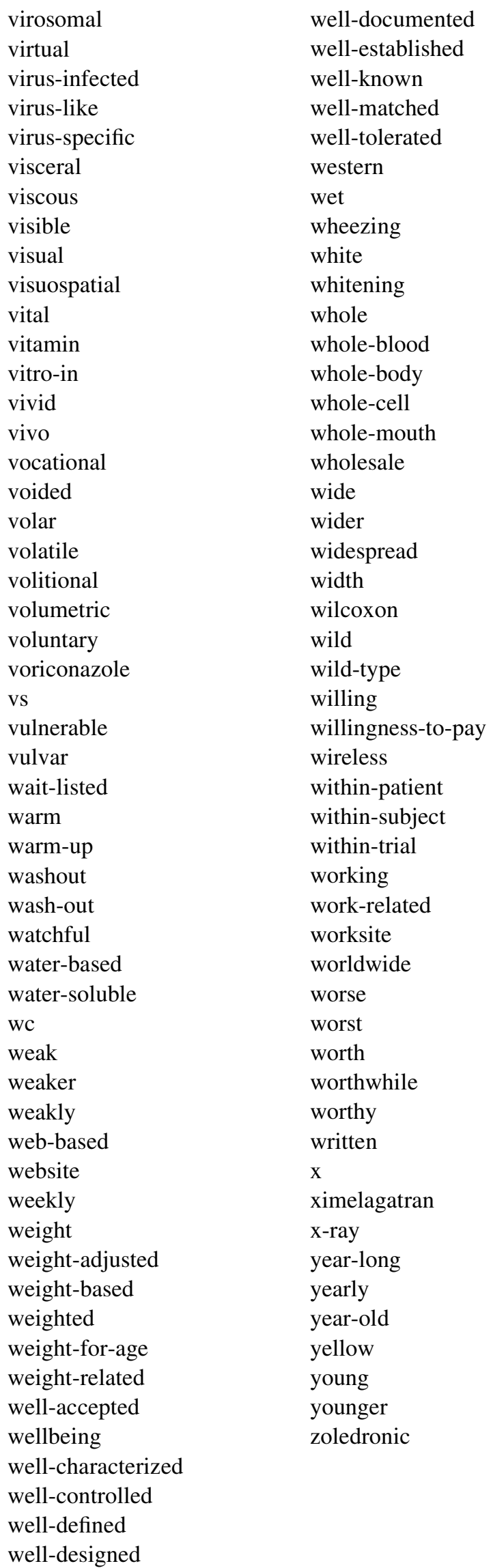

Appendix 3: Journals in which the abstracts were published, and number of abstracts by journal

\begin{tabular}{|c|c|}
\hline Journal & Count \\
\hline PloS one & 475 \\
\hline Journal of clinical pharmacology & 427 \\
\hline Trials & 344 \\
\hline BMC public health & 316 \\
\hline Diabetes care & 247 \\
\hline British journal of clinical pharmacology & 247 \\
\hline BMC musculoskeletal disorders & 225 \\
\hline Clinical therapeutics & 218 \\
\hline Current medical research and opinion & 186 \\
\hline British journal of cancer & 171 \\
\hline Vaccine & 147 \\
\hline Antimicrobial agents and chemotherapy & 143 \\
\hline $\begin{array}{l}\text { Journal of clinical oncology: official journal of the American } \\
\text { Society of Clinical Oncology }\end{array}$ & 143 \\
\hline Clinical pharmacology and therapeutics & 138 \\
\hline $\begin{array}{l}\text { International journal of clinical pharmacology and } \\
\text { therapeutics }\end{array}$ & 126 \\
\hline BMC psychiatry & 121 \\
\hline New England journal of medicine & 119 \\
\hline Lancet & 116 \\
\hline BMJ (Clinical research ed).) & 113 \\
\hline Diabetes, obesity and metabolism & 111 \\
\hline Journal of clinical psychiatry & 110 \\
\hline Critical care (London, England) & 109 \\
\hline Journal of clinical endocrinology and metabolism & 108 \\
\hline Annals of the rheumatic diseases & 107 \\
\hline BMC health services research & 107 \\
\hline BMC cancer & 102 \\
\hline European journal of clinical pharmacology & 99 \\
\hline Arthritis and rheumatism & 86 \\
\hline Clinical pharmacokinetics & 85 \\
\hline Nutrition journal & 83 \\
\hline Respiratory medicine & 79 \\
\hline Journal of infectious diseases & 78 \\
\hline Journal of clinical psychopharmacology & 77 \\
\hline Alimentary pharmacology and therapeutics & 77 \\
\hline Journal of medical Internet research & 76 \\
\hline BMC geriatrics & 75 \\
\hline Psychopharmacology & 74 \\
\hline International journal of clinical practice & 71 \\
\hline BMC family practice & 68 \\
\hline Pediatric infectious disease journal & 63 \\
\hline BMC pediatrics & 63 \\
\hline Respiratory research & 62 \\
\hline Health and quality of life outcomes & 61 \\
\hline BMC complementary and alternative medicine & 61 \\
\hline
\end{tabular}




Journal
American journal of cardiology
American heart journal
BMC neurology
Journal of rheumatology
Biopharmaceutics and drug disposition
Diabetic medicine: a journal of the British Diabetic
Association
Clinical infectious diseases: an official publication of the
Infectious Diseases Society of America
Malaria journal
Journal of antimicrobial chemotherapy
Journal of clinical dentistry
Clinical drug investigation
Arthritis research and therapy
BMC nephrology
Annals of oncology: official journal of the European Society
for Medical Oncology/ESMO
Osteoporosis international: a journal established as result of
cooperation between the European Foundation for
Osteoporosis and the National Osteoporosis Foundation of
the USA

Cephalalgia: an international journal of headache

BMC infectious diseases

Circulation

Lipids in health and disease

Journal of bone and mineral research: the official journal of the American Society for Bone and Mineral Research

American journal of gastroenterology

Ophthalmology

American journal of clinical nutrition

BMC medical education

Clinical cancer research: an official journal of the American Association for Cancer Research

Cancer chemotherapy and pharmacology

European heart journal

British journal of psychiatry: the journal of mental science

European respiratory journal

Journal of affective disorders

Journal of the American College of Cardiology

Pediatrics

International journal of chronic obstructive pulmonary disease

Biological psychiatry

BMC cardiovascular disorders

Neurology

BMC gastroenterology

European journal of cancer (Oxford, England: 1990)

BMC medicine

BMC pregnancy and childbirth

Diabetes technology and therapeutics

\section{Count}

\begin{tabular}{lc}
\hline Journal & Count \\
\hline Headache & 36 \\
Journal of international medical research & 35 \\
Schizophrenia research & 35 \\
Pharmacotherapy & 34 \\
Journal of acquired immune deficiency syndromes (1999) & 34 \\
British journal of nutrition & 34 \\
International journal of behavioral nutrition and physical & 34 \\
activity &
\end{tabular}

Diabetes $\quad 34$

American journal of psychiatry $\quad 34$

Yonsei medical journal $\quad 33$

Thorax 33

Annals of allergy, asthma and immunology: official 33 publication of the American College of Allergy, Asthma, and Immunology

Arzneimittel-Forschung $\quad 33$

American journal of dentistry 33

British journal of dermatology 33

BMC pulmonary medicine $\quad 32$

Chest $\quad 32$

Journal of nutrition $\quad 32$

Journal of Korean medical science $\quad 32$

BMC medical research methodology $\quad 32$

Journal of neuroscience: the official journal of the Society for 31 Neuroscience

American journal of respiratory and critical care medicine 31

Journal of cardiovascular pharmacology 31

Breast cancer research and treatment 31

Neuropsychopharmacology: official publication of the $\quad 30$ American College of Neuropsychopharmacology

BJU international $\quad 30$

Clinics (São Paulo, Brazil) $\quad 30$

Acta orthopaedica $\quad 30$

Diabetes research and clinical practice $\quad 30$

$\begin{array}{ll}\text { Critical care medicine } & 30\end{array}$

Journal of psychiatric research $\quad 30$

Human vaccines $\quad 30$

Journal of psychopharmacology (Oxford, England) 29

Gastroenterology 29

International clinical psychopharmacology 29

Journal of clinical hypertension (Greenwich, Conn.) 28

Cardiovascular diabetology $\quad 28$

Clinical journal of pain $\quad 28$

Pain $\quad 27$

Implementation science: IS $\quad 27$

Drug metabolism and disposition: the biological fate of 27 chemicals

Cancer $\quad 27$

Metabolism: clinical and experimental 27

Journal of allergy and clinical immunology 27 


\begin{tabular}{|c|c|}
\hline Journal & Coun \\
\hline Bone & 27 \\
\hline Rheumatology (Oxford, England) & 27 \\
\hline Journal of sexual medicine & 27 \\
\hline AIDS (London, England) & 27 \\
\hline Hypertension & 27 \\
\hline Thrombosis and haemostasis & 26 \\
\hline British journal of anaesthesia & 25 \\
\hline $\begin{array}{l}\text { Journal of strength and conditioning research/National } \\
\text { Strength and Conditioning Association }\end{array}$ & 25 \\
\hline Urology & 25 \\
\hline Annals of internal medicine & 25 \\
\hline Lancet. Oncology & 25 \\
\hline Stroke; a journal of cerebral circulation & 25 \\
\hline Journal of headache and pain & 24 \\
\hline $\begin{array}{l}\text { Value in health: the journal of the International Society for } \\
\text { Pharmacoeconomics and Outcomes Research }\end{array}$ & 24 \\
\hline Journal of cardiothoracic surgery & 24 \\
\hline Obesity (Silver Spring, Md.) & 24 \\
\hline European journal of applied physiology & 24 \\
\hline Pharmaceutical research & 24 \\
\hline Journal of the American Geriatrics Society & 24 \\
\hline Journal of urology & 24 \\
\hline Journal of cognitive neuroscience & 24 \\
\hline BMC surgery & 24 \\
\hline Journal of pain: official journal of the American Pain Society & 24 \\
\hline $\begin{array}{l}\text { Journal of thoracic oncology: official publication of the } \\
\text { International Association for the Study of Lung Cancer }\end{array}$ & 23 \\
\hline Contemporary clinical trials & 23 \\
\hline Journal of general internal medicine & 23 \\
\hline Journal of hypertension & 23 \\
\hline Arthritis care and research & 23 \\
\hline Scientificworldjournal & 22 \\
\hline $\begin{array}{l}\text { Health psychology: official journal of the Division of Health } \\
\text { Psychology, American Psychological Association }\end{array}$ & 22 \\
\hline Diabetologia & 22 \\
\hline Journal of human hypertension & 22 \\
\hline Medicine and science in sports and exercise & 22 \\
\hline Journal of the American Academy of Dermatology & 22 \\
\hline Spine & 22 \\
\hline European journal of clinical nutrition & 22 \\
\hline Clinical and vaccine immunology: CVI & 22 \\
\hline Preventive medicine & 22 \\
\hline American journal of tropical medicine and hygiene & 22 \\
\hline Anesthesia and analgesia & 22 \\
\hline Digestive diseases and sciences & 22 \\
\hline Journal of child and adolescent psychopharmacology & 21 \\
\hline Menopause (New York, N.Y.) & 21 \\
\hline Journal of clinical epidemiology & 21 \\
\hline $\begin{array}{l}\text { International journal of cancer. Journal international du } \\
\text { cancer }\end{array}$ & 21 \\
\hline
\end{tabular}

\begin{tabular}{lc}
\hline Journal & Cou \\
\hline European journal of heart failure & 21 \\
Journal of pharmaceutical sciences & 21 \\
BMC medical informatics and decision making & 21 \\
Nephrology, dialysis, transplantation: official publication of & 21
\end{tabular}

Chrology, dialysis, transplantation: official publication of -21 the European Dialysis and Transplant AssociationEuropean Renal Association

Blood

Hepatology (Baltimore, Md.) 20

World journal of surgery 20

Heart (British Cardiac Society) 20

Vascular health and risk management $\quad 20$

Human psychopharmacology 20

JAMA 20

Archives of internal medicine $\quad 20$

$\begin{array}{ll}\text { Patient education and counseling } & 19\end{array}$

Pain medicine (Malden, Mass.) 19

Clinical journal of the American Society of Nephrology: 19 CJASN

HIV clinical trials $\quad 19$

Nicotine and tobacco research: official journal of the Society 19 for Research on Nicotine and Tobacco

American journal of therapeutics 19

Intensive care medicine $\quad 19$

Clinical interventions in aging $\quad 19$

American journal of hypertension $\quad 19$

Lancet. Neurology 19

Fertility and sterility 18

Contraception 18

$\begin{array}{ll}\text { Pulmonary pharmacology and therapeutics } & 18\end{array}$

Human reproduction (Oxford, England) 18

Epilepsia $\quad 18$

$\begin{array}{ll}\text { Atherosclerosis } & 18\end{array}$

Psychoneuroendocrinology 18

Journal of asthma: official journal of the Association for the 17 Care of Asthma

Allergy and asthma proceedings: the official journal of $\quad 17$ regional and state allergy societies

PharmacoEconomics 17

Obstetrics and gynecology 17

International journal of obesity (2005) 17

Postgraduate medicine $\quad 17$

$\begin{array}{ll}\text { Journal of diabetes science and technology } & 17\end{array}$

Journal of bone and joint surgery. American volume 17

Transplantation $\quad 17$

BMC clinical pharmacology 17

Reproductive biology and endocrinology: RB\&E 17

BMC ophthalmology 16

Drugs in R\&D 16

BMC research notes 16

European urology 16 


\begin{tabular}{|c|c|}
\hline Journal & Coun \\
\hline Kidney international & 16 \\
\hline $\begin{array}{l}\text { American journal of kidney diseases: the official journal of } \\
\text { the National Kidney Foundation }\end{array}$ & 16 \\
\hline $\begin{array}{l}\text { Quality of life research: an international journal of quality of } \\
\text { life aspects of treatment, care and rehabilitation }\end{array}$ & 16 \\
\hline $\begin{array}{l}\text { British journal of general practice: the journal of the Royal } \\
\text { College of General Practitioners }\end{array}$ & 16 \\
\hline Appetite & 16 \\
\hline Oncologist & 16 \\
\hline American journal of medicine & 16 \\
\hline $\begin{array}{l}\text { Medical science monitor: international medical journal of } \\
\text { experimental and clinical research }\end{array}$ & 16 \\
\hline Journal of the American College of Nutrition & 16 \\
\hline PLoS neglected tropical diseases & 16 \\
\hline $\begin{array}{l}\text { Journal of alternative and complementary medicine (New } \\
\text { York, NY) }\end{array}$ & 16 \\
\hline Nutrients & 16 \\
\hline Surgical endoscopy & 16 \\
\hline Psychopharmacology bulletin & 15 \\
\hline Human vaccines and immunotherapeutics & 15 \\
\hline Psychiatry research & 15 \\
\hline Archives of general psychiatry & 15 \\
\hline British journal of ophthalmology & 15 \\
\hline Journal of pediatrics & 15 \\
\hline European journal of anaesthesiology & 15 \\
\hline Journal of periodontology & 15 \\
\hline International journal of cardiology & 15 \\
\hline Clinical rehabilitation & 14 \\
\hline Multiple sclerosis (Houndmills, Basingstoke, England) & 14 \\
\hline Annals of pharmacotherapy & 14 \\
\hline International journal of geriatric psychiatry & 14 \\
\hline Expert opinion on pharmacotherapy & 14 \\
\hline Environmental health perspectives & 14 \\
\hline Journal of pharmacology and experimental therapeutics & 14 \\
\hline Addictive behaviors & 14 \\
\hline $\begin{array}{l}\text { American journal of transplantation: official journal of the } \\
\text { American Society of Transplantation and the American } \\
\text { Society of Transplant Surgeons }\end{array}$ & 14 \\
\hline Gut & 14 \\
\hline $\begin{array}{l}\text { European neuropsychopharmacology: the journal of the } \\
\text { European College of Neuropsychopharmacology }\end{array}$ & 14 \\
\hline PLoS medicine & 14 \\
\hline Thrombosis research & 14 \\
\hline $\begin{array}{l}\text { Journal of the American Academy of Child and Adolescent } \\
\text { Psychiatry }\end{array}$ & 14 \\
\hline AIDS research and human retroviruses & 13 \\
\hline American journal of obstetrics and gynecology & 13 \\
\hline Archives of ophthalmology & 13 \\
\hline Experimental brain research & 13 \\
\hline American journal of surgery & 13 \\
\hline
\end{tabular}

\begin{tabular}{lc}
\hline Journal Count \\
\hline
\end{tabular}

Anesthesiology 13

International journal of antimicrobial agents $\quad 13$

Lung cancer (Amsterdam, Netherlands) 13

Cancer prevention research (Philadelphia, Pa.) 13

Medicina oral, patologí-a oral y cirugí-a bucal 13

Hormone and metabolic research $=$ Hormon- und 13

Stoffwechselforschung $=$ Hormones et métabolisme

Acta anaesthesiologica Scandinavica $\quad 13$

International journal of medical sciences $\quad 13$

Cancer epidemiology, biomarkers and prevention: a $\quad 13$ publication of the American Association for Cancer Research, cosponsored by the American Society of Preventive Oncology

$\begin{array}{ll}\text { Cardiovascular ultrasound } & 13\end{array}$

British journal of surgery $\quad 13$

Calcified tissue international $\quad 13$

Journal of gastroenterology and hepatology 12

International journal of gynaecology and obstetrics: the 12 official organ of the International Federation of Gynaecology and Obstetrics

Journal of thrombosis and haemostasis: JTH 12

Allergy 12

Behaviour research and therapy 12

AIDS and behavior $\quad 12$

American journal of physiology. Endocrinology and 12 metabolism

$\begin{array}{lr}\text { Anaesthesia } & 12\end{array}$

Investigative ophthalmology and visual science $\quad 12$

European journal of endocrinology/European Federation of 12 Endocrine Societies

Archives of pediatrics and adolescent medicine $\quad 12$

Circulation journal: official journal of the Japanese 12 Circulation Society

Paediatric anaesthesia $\quad 12$

Journal of neurophysiology $\quad 12$

Compendium of continuing education in dentistry $\quad 12$ (Jamesburg, NJ: 1995)

British journal of sports medicine $\quad 12$

European spine journal: official publication of the European 12 Spine Society, the European Spinal Deformity Society, and the European Section of the Cervical Spine Research Society

Investigational new drugs $\quad 12$

American journal of health-system pharmacy: AJHP: official 12 journal of the American Society of Health-System Pharmacists

BMC women's health $\quad 12$

Journal of drugs in dermatology: JDD 12

Archives of physical medicine and rehabilitation $\quad 12$

Health education research $\quad 11$

Journal of the National Cancer Institute 11

Journal of neuroengineering and rehabilitation 11 


\begin{tabular}{|c|c|c|c|}
\hline Journal & Count & Journal & Count \\
\hline Advances in therapy & 11 & \multirow{2}{*}{$\begin{array}{l}\text { Dermatologic surgery: official publication for American } \\
\text { Society for Dermatologic Surgery [et al.] }\end{array}$} & \multirow[t]{2}{*}{10} \\
\hline Korean journal of ophthalmology: KJO & 11 & & \\
\hline Radiation oncology (London, England) & 11 & Upsala journal of medical sciences & 10 \\
\hline Antiviral therapy & 11 & \multirow{2}{*}{$\begin{array}{l}\text { Otolaryngology-head and neck surgery: official journal of } \\
\text { American Academy of Otolaryngology-Head and Neck } \\
\text { Surgery }\end{array}$} & \multirow[t]{2}{*}{10} \\
\hline Pharmacogenetics and genomics & 11 & & \\
\hline American journal of ophthalmology & 11 & Drug and alcohol dependence & 10 \\
\hline Journal of consulting and clinical psychology & 11 & European journal of nutrition & 10 \\
\hline Journal of bone and joint surgery. British volume & 11 & Vision research & 10 \\
\hline Bone marrow transplantation & 11 & \multirow{2}{*}{$\begin{array}{l}\text { Cardiovascular drugs and therapy/sponsored by the } \\
\text { International Society of Cardiovascular Pharmacotherapy }\end{array}$} & \multirow[t]{2}{*}{10} \\
\hline Journal of dermatological treatment & 11 & & \\
\hline Psychological medicine & 11 & Lancet. Infectious diseases & 10 \\
\hline \multirow{2}{*}{$\begin{array}{l}\text { European journal of clinical microbiology and infectious } \\
\text { diseases: official publication of the European Society of } \\
\text { Clinical Microbiology }\end{array}$} & \multirow[t]{2}{*}{11} & Nutrition (Burbank, Los Angeles County, Calif.) & 10 \\
\hline & & International psychogeriatrics/IPA & 10 \\
\hline World journal of surgical oncology & 11 & Journal of nutrition education and behavior & 10 \\
\hline Maturitas & 11 & European journal of pain (London, England) & 10 \\
\hline BMC emergency medicine & 11 & Journal of neurology & 10 \\
\hline Journal of the American Society of Hypertension: JASH & 11 & Clinical pediatrics & 9 \\
\hline Age and ageing & 11 & $\begin{array}{l}\text { Climacteric: the journal of the International Menopause } \\
\text { Society }\end{array}$ & 9 \\
\hline Bipolar disorders & 10 & Circulation. Cardiovascular genetics & 9 \\
\hline $\begin{array}{l}\text { Journal of adolescent health: official publication of the } \\
\text { Society for Adolescent Medicine }\end{array}$ & 10 & $\begin{array}{l}\text { Journal of cardiovascular magnetic resonance: official } \\
\text { journal of the Society for Cardiovascular Magnetic }\end{array}$ & \multirow[t]{2}{*}{9} \\
\hline \multirow{2}{*}{$\begin{array}{l}\text { European journal of neurology: the official journal of the } \\
\text { European Federation of Neurological Societies }\end{array}$} & 10 & Resonance & \\
\hline & \multirow{2}{*}{10} & Clinical neuropharmacology & 9 \\
\hline $\begin{array}{l}\text { International journal of sport nutrition and exercise } \\
\text { metabolism }\end{array}$ & & $\begin{array}{l}\text { International journal of neuropsychopharmacology/official } \\
\text { scientific journal of the Collegium Internationale }\end{array}$ & 9 \\
\hline Drugs & 10 & Neuropsychopharmacologicum (CINP) & \\
\hline $\begin{array}{l}\text { Supportive care in cancer: official journal of the } \\
\text { Multinational Association of Supportive Care in Cancer }\end{array}$ & 10 & Journal of clinical lipidology & 9 \\
\hline $\begin{array}{l}\text { Multinational Association of Supportive Care in Cancer } \\
\text { Arteriosclerosis, thrombosis, and vascular biology }\end{array}$ & 10 & $\begin{array}{l}\text { Scandinavian journal of trauma, resuscitation and emergency } \\
\text { medicine }\end{array}$ & 9 \\
\hline Journal of anesthesia & 10 & COPD & 9 \\
\hline Annals of behavioral medicine: a publication of the Society & 10 & Clinical and experimental rheumatology & 9 \\
\hline & & Journal of pharmacokinetics and pharmacodynamics & 9 \\
\hline Journal of Alzheimer's disease: JAD & 10 & International journal of pharmaceutics & 9 \\
\hline Journal of hepatology & 10 & Journal of opioid management & 9 \\
\hline Clinical nutrition (Edinburgh, Scotland) & 10 & Journal of translational medicine & 9 \\
\hline Journal of pediatric gastroenterology and nutrition & 10 & Gastrointestinal endoscopy & 9 \\
\hline Journal of attention disorders & 10 & Sexually transmitted diseases & 9 \\
\hline $\begin{array}{l}\text { Osteoarthritis and cartilage/OARS, Osteoarthritis Research } \\
\text { Society }\end{array}$ & 10 & $\begin{array}{l}\text { Clinical and experimental allergy: journal of the British } \\
\text { Society for Allergy and Clinical Immunology }\end{array}$ & 9 \\
\hline Pharmacopsychiatry & 10 & Journal of contemporary dental practice & 9 \\
\hline Depression and anxiety & 10 & Scandinavian journal of gastroenterology & 9 \\
\hline Chemotherapy & 10 & American journal of sports medicine & 9 \\
\hline Journal of cardiac failure & 10 & Journal of clinical pharmacy and therapeutics & 9 \\
\hline World journal of gastroenterology: WJG & 10 & Journal of the American Dietetic Association & 9 \\
\hline Neuropsychologia & 10 & Japanese journal of clinical oncology & 9 \\
\hline $\begin{array}{l}\text { Diabetes and vascular disease research: official journal of the } \\
\text { International Society of Diabetes and Vascular Disease }\end{array}$ & 10 & Public health nutrition & 9 \\
\hline Progress in neuro-psychopharmacology and biological & 10 & Journal of autism and developmental disorders & 9 \\
\hline
\end{tabular}




Journal
Clinical gastroenterology and hepatology: the official clinical
practice journal of the American Gastroenterological
Association
Asian Pacific journal of cancer prevention: APJCP
Journal of arthroplasty
Biological psychology
Hypertension research: official journal of the Japanese
Society of Hypertension
European journal of pharmaceutical sciences: official journal
of the European Federation for Pharmaceutical Sciences
British journal of health psychology
Sexually transmitted infections
Journal of ocular pharmacology and therapeutics: the official
journal of the Association for Ocular Pharmacology and
Therapeutics
Indian journal of ophthalmology
American journal of geriatric psychiatry: official journal of
the American Association for Geriatric Psychiatry
Eye (London, England)
Reproductive biomedicine online
Journal of neurology, neurosurgery, and psychiatry
Europace: European pacing, arrhythmias, and cardiac
electrophysiology: journal of the working groups on
cardiac pacing, arrhythmias, and cardiac cellular
electrophysiology of the European Society of Cardiology
Archives of disease in childhood
Diabetes/metabolism research and reviews
BJOG: an international journal of obstetrics and gynaecology
Cancer causes and control: CCC
European journal of cardiovascular prevention and
rehabilitation: official journal of the European Society of
Cardiology, Working Groups on Epidemiology and
Prevention and Cardiac Rehabilitation and Exercise
Physiology

Infectious diseases in obstetrics and gynecology

Nutrition research (New York, NY)

Tropical medicine and international health: TM and IH

Journal of lipid research

Addiction (Abingdon, England)

Pediatric pulmonology

Clinical oral investigations

Cardiology

Journal of child psychology and psychiatry, and allied disciplines

American journal of preventive medicine

Knee surgery, sports traumatology, arthroscopy: official journal of the ESSKA

Proceedings of the National Academy of Sciences of the United States of America

Clinical endocrinology

Epilepsy and behavior: E\&B

Clinical cardiology

\begin{tabular}{ll}
\hline Journal Count \\
\hline
\end{tabular}

Acupuncture in medicine: journal of the British Medical

Acupuncture Society

International journal of colorectal disease $\quad 8$

Inflammatory bowel diseases $\quad 8$

Dermatology (Basel, Switzerland) $\quad 8$

International journal of nursing studies $\quad 8$

Journal of perinatology: official journal of the California 8

Perinatal Association

Substance abuse treatment, prevention, and policy $\quad 8$

Pharmacology $\quad 8$

British journal of haematology $\quad 8$

Experimental and clinical endocrinology and diabetes: 8 official journal, German Society of Endocrinology [and] German Diabetes Association

Chinese medical journal 7

$\begin{array}{ll}\text { European journal of medical research } & 7\end{array}$

Journal of orthopaedic and sports physical therapy $\quad 7$

$\begin{array}{ll}\text { Perception } & 7\end{array}$

International orthopaedics $\quad 7$

European journal of contraception and reproductive health 7 care: the official journal of the European Society of Contraception

$\begin{array}{ll}\text { Breast cancer research: BCR } & 7\end{array}$

$\begin{array}{ll}\text { Journal of clinical nursing } & 7\end{array}$

$\begin{array}{ll}\text { BMC neuroscience } & 7\end{array}$

$\begin{array}{ll}\text { Behavioral and brain functions: BBF } & 7\end{array}$

International journal of sports medicine $\quad 7$

$\begin{array}{ll}\text { Brain: a journal of neurology } & 7\end{array}$

Psychology and health $\quad 7$

Journal of occupational and environmental medicine/ $\quad 7$ American College of Occupational and Environmental Medicine

$\begin{array}{ll}\text { Science translational medicine } & 7\end{array}$

International journal of impotence research $\quad 7$

$\begin{array}{ll}\text { Journal of occupational rehabilitation } & 7\end{array}$

$\begin{array}{ll}\text { Surgical infections } & 7\end{array}$

Journal of the American Society of Nephrology: JASN 7

Arthroscopy: the journal of arthroscopic and related surgery: 7 official publication of the Arthroscopy Association of

North America and the International Arthroscopy

Association

European journal of obstetrics, gynecology, and reproductive $\quad 7$ biology

$\begin{array}{ll}\text { Annals of surgery } & 7\end{array}$

Journal of sports sciences $\quad 7$

Journal of oral and maxillofacial surgery: official journal of 7 the American Association of Oral and Maxillofacial Surgeons

Head and face medicine $\quad 7$

$\begin{array}{ll}\text { Basic and clinical pharmacology and toxicology } & 7\end{array}$

$\begin{array}{ll}\text { Pediatric research } & 7\end{array}$

$\begin{array}{ll}\text { Journal of evaluation in clinical practice } & 7\end{array}$ 


Journal Count

American journal of cardiovascular drugs: drugs, devices, and other interventions

Journal of the European Academy of Dermatology and Venereology: JEADV

European journal of cardio-thoracic surgery: official journal of the European Association for Cardio-thoracic Surgery

Movement disorders: official journal of the Movement Disorder Society

Sleep medicine

American journal of managed care

HPB surgery: a world journal of hepatic, pancreatic and biliary surgery

Blood pressure monitoring

Gynecologic oncology

International journal of radiation oncology, biology, physics

Journal of behavioral medicine

Dementia and geriatric cognitive disorders

Journal of cardiothoracic and vascular anesthesia

Occupational and environmental medicine

Asia Pacific journal of clinical nutrition

Clinical orthopaedics and related research

Minerva anestesiologica

Journal of the Association of Physicians of India

Mayo Clinic proceedings

Journals of gerontology. Series A, Biological sciences and medical sciences

BMC dermatology

Canadian journal of anaesthesia $=$ Journal canadien d'anesthésie

Sleep

Platelets

Journal of clinical anesthesia

Journal of epidemiology and community health

BMC urology

Pain practice: the official journal of World Institute of Pain Journal of internal medicine

Pharmacogenomics journal

Translational psychiatry

NeuroImage

Phytomedicine: international journal of phytotherapy and phytopharmacology

Journal of applied physiology (Bethesda, Md.: 1985)

European journal of clinical investigation

Retina (Philadelphia, Pa.)

Journal of endocrinological investigation

Neurourology and urodynamics

Scandinavian journal of clinical and laboratory investigation

Physiology and behavior

Neuropharmacology

Acta oncologica (Stockholm, Sweden)

Journal Count

Journal of endourology/Endourological Society

Applied physiology, nutrition, and

metabolism $=$ Physiologie appliquée, nutrition et métabolisme

Oncology

Scandinavian journal of work, environment and health

Journal of manipulative and physiological therapeutics

International journal of infectious diseases: IJID: official publication of the International Society for Infectious Diseases

International urology and nephrology

Journal of aerosol medicine: the official journal of the International Society for Aerosols in Medicine

Journal of the American Heart Association

Anti-cancer drugs

Acta paediatrica (Oslo, Norway: 1992)

Transactions of the Royal Society of Tropical Medicine and Hygiene

Journal of glaucoma 6

Annals of surgical oncology

Oral surgery, oral medicine, oral pathology, oral radiology, 6 and endodontics

BMC oral health

Clinical trials (London, England)

Journal of bone and mineral metabolism

Therapeutic drug monitoring

Mediators of inflammation

Cerebrovascular diseases (Basel, Switzerland)

Respirology (Carlton, Vic.)

Family practice

Journal of health, population, and nutrition

Journal of pharmacy and pharmacology

Scandinavian journal of gastroenterology. Supplement

Journal of anxiety disorders

Journal of women's health (2002)

Medical care

Epilepsy research

Journal of the Indian Medical Association

Journal of viral hepatitis

Medical decision making: an international journal of the Society for Medical Decision Making

Journal of the American Medical Directors Association 6

Neuropsychobiology 6

Paediatric drugs 6

Haematologica 6

Journal of diabetes and its complications $\quad 6$

Acta psychiatrica Scandinavica 6

Journal of behavior therapy and experimental psychiatry 6

Annals of the New York Academy of Sciences 6

Angle orthodontist

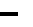




\begin{tabular}{|c|c|c|c|}
\hline Journal & Count & Journal & Count \\
\hline Journal of gastroenterology & 6 & Diagnostic microbiology and infectious disease & 5 \\
\hline Indian journal of medical research & 6 & \multirow{2}{*}{$\begin{array}{l}\text { Pediatric allergy and immunology: official publication of the } \\
\text { European Society of Pediatric Allergy and Immunology }\end{array}$} & 5 \\
\hline $\begin{array}{l}\text { American journal of physical medicine and rehabilitation/ } \\
\text { Association of Academic Physiatrists }\end{array}$ & 6 & & 5 \\
\hline Laryngoscope & 6 & Archives of neurology & 5 \\
\hline Clinical breast cancer & 6 & Journal of negative results in biomedicine & 5 \\
\hline $\begin{array}{l}\text { Journal of experimental psychology. Human perception and } \\
\text { performance }\end{array}$ & 6 & $\begin{array}{l}\text { Journal of stroke and cerebrovascular diseases: the official } \\
\text { journal of National Stroke Association }\end{array}$ & 5 \\
\hline Hepato-gastroenterology & 6 & European journal of drug metabolism and pharmacokinetics & 5 \\
\hline Journal of pain and symptom management & 6 & Journal of ECT & 5 \\
\hline Current Alzheimer research & 6 & AAPS journal & 5 \\
\hline Journal of experimental and clinical cancer research: CR & 6 & Journal of the neurological sciences & 5 \\
\hline Molecular psychiatry & 6 & Journal of chemotherapy (Florence, Italy) & 5 \\
\hline Journal of neural transmission (Vienna, Austria: 1996) & 6 & Clinical science (London, England: 1979) & 5 \\
\hline Journal of cataract and refractive surgery & 6 & Canadian journal of cardiology & 5 \\
\hline Community dentistry and oral epidemiology & 6 & Journal of traditional Chinese medicine $=$ Chung i tsa chih & 5 \\
\hline Pacing and clinical electrophysiology: PACE & 6 & sored by All-China Associat & \\
\hline European journal of dermatology: EJD & 6 & $\begin{array}{l}\text { Iraditional Cninese Medicine, Academy of I raditional } \\
\text { Chinese Medicine }\end{array}$ & \\
\hline Psychological research & 6 & Journal of psychosomatic research & 5 \\
\hline $\begin{array}{l}\text { EuroIntervention: journal of EuroPCR in collaboration with } \\
\text { the Working Group on Interventional Cardiology of the }\end{array}$ & 6 & Blood pressure & 5 \\
\hline European Society of Cardiology & & $\begin{array}{l}\text { Journal of the Medical Association of } \\
\text { Thailand = Chotmaihet thangphaet }\end{array}$ & 5 \\
\hline $\begin{array}{l}\text { Annals of clinical psychiatry: official journal of the } \\
\text { American Academy of Clinical Psychiatrists }\end{array}$ & 6 & $\begin{array}{l}\text { Neurogastroenterology and motility: the official journal of } \\
\text { the European Gastrointestinal Motility Society }\end{array}$ & 5 \\
\hline & 5 & Saudi medical journal & 5 \\
\hline physiology & J & Pediatric diabetes & 5 \\
\hline International journal of clinical pharmacology research & 5 & International journal of dermatology & 5 \\
\hline $\begin{array}{l}\text { Journal of clinical sleep medicine: JCSM: official publication } \\
\text { of the American Academy of Sleep Medicine }\end{array}$ & 5 & $\begin{array}{l}\text { Endocrine practice: official journal of the American College } \\
\text { of Endocrinology and the American Association of Clinical } \\
\text { Endocrinologists }\end{array}$ & 5 \\
\hline American journal of hematology & 5 & Alzheimer disease and associated disorders & 5 \\
\hline $\begin{array}{l}\text { Radiotherapy and oncology: journal of the European Society } \\
\text { for Therapeutic Radiology and Oncology }\end{array}$ & 5 & Journal of thrombosis and thrombolysis & 5 \\
\hline Pharmaceutical statistics & 5 & Parkinsonism and related disorders & 5 \\
\hline AIDS patient care and STDs & 5 & Health communication & 5 \\
\hline Psychotherapy and psychosomatics & 5 & Lasers in medical science & 5 \\
\hline Helicobacter & 5 & Journal of the American Dental Association (1939) & 5 \\
\hline British journal of pharmacology & 5 & International dental journal & 5 \\
\hline Graefe's archive for clinical and experimental & 5 & Psychiatric services (Washington, DC) & 5 \\
\hline $\begin{array}{l}\text { ophthalmology = Albrecht von Graefes Archiv für } \\
\text { klinische und experimentelle Ophthalmologie }\end{array}$ & & $\begin{array}{l}\text { Journal of gastrointestinal surgery: official journal of the } \\
\text { Society for Surgery of the Alimentary Tract }\end{array}$ & 5 \\
\hline Photomedicine and laser surgery & 5 & Journal of orthopaedics and traumatology: official journal of & 5 \\
\hline Operative dentistry & 5 & and Traum & \\
\hline European psychiatry: the journal of the Association of & 5 & Schizophrenia bulletin & 5 \\
\hline European Psychiatrists & & Clinical and applied thrombosis/hemostasis: official journal & 5 \\
\hline Cerebral cortex (New York, NY: 1991) & 5 & Academy of Clinical and Applied & \\
\hline European journal of gastroenterology and hepatology & 5 & 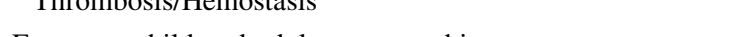 & \\
\hline Journal of hematology and oncology & 5 & European child and adolescent psychiatry & 5 \\
\hline Journal of thoracic and cardiovascular surgery & 5 & Clinics in orthopedic surgery & 5 \\
\hline Korean journal of radiology: official journal of the Korean & 5 & Particle and fibre toxicology & 5 \\
\hline Radiological Society & & Drug design, development and therapy & 5 \\
\hline
\end{tabular}




Journal
Physical therapy
Statistics in medicine
Journal of interventional cardiology
Journal of endodontics
Sleep and breathing = Schlaf \& Atmung
Emotion (Washington, DC)
Journal of vascular surgery
Middle East journal of anaesthesiology
European journal of vascular and endovascular surgery:
official journal of the European Society for Vascular
Surgery
Journal of clinical gastroenterology
Gynecological endocrinology: the official journal of the
International Society of Gynecological Endocrinology

International journal of technology assessment in health care

HIV medicine

Journal of virology

Regional anesthesia and pain medicine

Journal of pharmacokinetics and biopharmaceutics

Resuscitation

Skin pharmacology and physiology

Saudi journal of gastroenterology: official journal of the

Saudi Gastroenterology Association

Scandinavian journal of rheumatology. Supplement

Fundamental and clinical pharmacology

European journal of preventive cardiology

Aging and mental health

Journal of clinical periodontology

Journal of biopharmaceutical statistics

Health education and behavior: the official publication of the

Society for Public Health Education

Journal of dentistry

European radiology

Journal of the American Medical Informatics Association: JAMIA

Journal of studies on alcohol and drugs

Seminars in arthritis and rheumatism

Psychosomatic medicine

Circulation. Cardiovascular quality and outcomes

Palliative medicine

Journal of dental research

Journal of immunotherapy (Hagerstown, Md: 1997)

Medical journal of Australia

CMAJ: Canadian Medical Association journal $=$ journal de

l'Association medicale canadienne

International archives of allergy and immunology

Gait and posture

AJNR. American journal of neuroradiology

Transplantation proceedings

Child: care, health and development
Count

5

5

5

5

5

5

5

5

5

5

\section{5}

5

5

5

4

4

4

4

4

4

4

4

4

4

4

4

4

4

4

4

4

4

4

4

4

4

4

4

4

4

4

4
Journal

Count

Clinical microbiology and infection: the official publication

of the European Society of Clinical Microbiology and

Infectious Diseases

International journal of clinical pharmacology, therapy, and 4 toxicology

Journal of abnormal child psychology

Archives of dermatological research

Emergency medicine journal: EMJ

Journal of pediatric endocrinology and metabolism: JPEM

Clinical lung cancer

Transplant international: official journal of the European Society for Organ Transplantation

Nutrition, metabolism, and cardiovascular diseases: NMCD 4

International journal of epidemiology

Respiratory physiology and neurobiology

Journal of cystic fibrosis: official journal of the European Cystic Fibrosis Society

Gynecologic and obstetric investigation

European journal of haematology

Journal of diabetes

Clinical and experimental pharmacology and physiology

International journal of psychophysiology: official journal of the International Organization of Psychophysiology

Clinical and experimental hypertension (New York, NY: 4 1993)

Allergology international: official journal of the Japanese 4 Society of Allergology

European archives of oto-rhino-laryngology: official journal of the European Federation of Oto-Rhino-Laryngological Societies (EUFOS): affiliated with the German Society for Oto-Rhino-Laryngology—Head and Neck Surgery

Acta psychologica

Seminars in oncology

Scandinavian journal of public health

European neurology

Molecular vision

Journal of cardiovascular nursing

Infection

Journal of atherosclerosis and thrombosis

Scandinavian journal of medicine and science in sports

American journal of geriatric pharmacotherapy

International journal of stroke: official journal of the International Stroke Society

Ophthalmologica. Journal international d'ophtalmologie. International journal of ophthalmology. Zeitschrift für Augenheilkunde

Journal of the Formosan Medical Association = Taiwan yi 4 zhi

Journal of cosmetic dermatology 4

Psychology, health and medicine 4

International urogynecology journal 4

American journal of infection control
4

4

4

4

4

4

4

4

4

4

4

4

4 . 4 


Journal
American journal of rhinology and allergy
Scandinavian journal of urology and nephrology
Techniques in coloproctology
Spine journal: official journal of the North American Spine
Society
Anticancer research
Journal of cardiovascular pharmacology and therapeutics
Blood coagulation and fibrinolysis: an international journal in
haemostasis and thrombosis

Annals of thoracic surgery

Lupus

Leukemia

Clinical biomechanics (Bristol, Avon)

European journal of pediatrics

Neuroscience letters

BMC medical genetics

Circulation. Arrhythmia and electrophysiology

Pain physician

Rheumatology international

Science (New York, NY)

Neurobiology of learning and memory

Antiviral research

Expert opinion on investigational drugs

Military medicine

Oncology reports

Metabolic syndrome and related disorders

Journal of substance abuse treatment

Regulatory toxicology and pharmacology: RTP

Brain, behavior, and immunity

Archives of dermatology

International journal of behavioral medicine

Journal of medicinal food

Alcoholism, clinical and experimental research

Acta neurochirurgica. Supplement

Current HIV research

Journal of cardiovascular electrophysiology

Clinical rheumatology

Scandinavian journal of primary health care

Journal of physiotherapy

Australian and New Zealand journal of psychiatry

Southeast Asian journal of tropical medicine and public health

JACC. Cardiovascular interventions

Journal of magnetic resonance imaging: JMRI

Health technology assessment (Winchester, England)

Clinical physiology and functional imaging

Regulatory peptides

Clinical transplantation

European journal of ophthalmology
Count

4

4

4

4

4

4

4

4

4

4

4

4

4

4

4

4

4

4

4

4

4

4

4

4

4

4

4

4

4

4

4

4

4

4

4

4

4

4

4

4

4

4

4

4

4

4

4

\begin{tabular}{lc}
\hline Journal & Count \\
\hline
\end{tabular}

Nursing research

Journal of the Indian Society of Pedodontics and Preventive 4 Dentistry

Artificial organs 4

Journal of rehabilitation medicine 4

International urogynecology journal and pelvic floor $\quad 4$ dysfunction

Transfusion 4

Muscle and nerve 4

Applied psychophysiology and biofeedback 4

Orthopedics 4

Knee 4

International journal of obesity and related metabolic 4 disorders: journal of the International Association for the Study of Obesity

Circulation. Cardiovascular interventions 4

Chinese journal of integrative medicine 4

Journal of physical activity and health 4

Modern rheumatology/the Japan Rheumatism Association 4

Journal of child neurology 4

Archives of orthopaedic and trauma surgery 4

Anaesthesia and intensive care 4

Clinical toxicology (Philadelphia, Pa.) 4

AIDS care 4

American journal of Alzheimer's disease and other 4 dementias

Pediatric nephrology (Berlin, Germany) 4

Journal of medical economics 4

Cancer research 4

Pharmacological reports: PR 4

Injury prevention: journal of the International Society for 3 Child and Adolescent Injury Prevention

Neurorehabilitation and neural repair $\quad 3$

Annals of nutrition and metabolism 3

Skin research and technology: official journal of International 3 Society for Bioengineering and the Skin (ISBS) [and] International Society for Digital Imaging of Skin (ISDIS) [and] International Society for Skin Imaging (ISSI)

Journal of the American Board of Family Medicine: JABFM 3

Journal of clinical immunology 3

Journal of telemedicine and telecare $\quad 3$

American journal of nephrology 3

Health expectations: an international journal of public 3 participation in health care and health policy

Social science and medicine (1982) 3

Respiration; international review of thoracic diseases 3

Journal of cosmetic science 3

BMC medical ethics 3

European journal of pediatric surgery: official journal of 3 Austrian Association of Pediatric Surgery... [et al. $]=$ Zeitschrift für Kinderchirurgie 


Journal
Annals of plastic surgery
CNS spectrums
Journal of immunology (Baltimore, Md: 1950)
Internal medicine (Tokyo, Japan)
Pediatric cardiology
Analytical chemistry
Journal of dental hygiene: JDH/American Dental Hygienists'
Association
Postgraduate medical journal
Annals of Saudi medicine
Cutaneous and ocular toxicology
AAPS pharmSci
Nephrology (Carlton, Vic.)
Archives of gynecology and obstetrics
Familial cancer
Forschende Komplementärmedizin (2006)
Growth hormone and IGF research: official journal of the
Growth Hormone Research Society and the International
IGF Research Society

Oxidative medicine and cellular longevity

Inflammopharmacology

Diabetes and metabolism

Medical oncology (Northwood, London, England)

European journal of radiology

Canadian respiratory journal: journal of the Canadian

Thoracic Society

Journal of supportive oncology

Complementary therapies in medicine

Pharmacogenomics

Microvascular research

European archives of psychiatry and clinical neuroscience

Archives of surgery (Chicago, Ill.: 1960)

Endoscopy

Contact dermatitis

Lasers in surgery and medicine

European journal of orthodontics

Behavioural pharmacology

Hong Kong medical journal = Xianggang yi xue za zhi/

Hong Kong Academy of Medicine

Journal of clinical microbiology

Journal of nutrition, health and aging

Neurosurgery

Journal of advanced nursing

Journal of orthopaedic surgery and research

Acta anaesthesiologica Belgica

Neurobiology of aging

International journal of gynecological cancer: official journal of the International Gynecological Cancer Society

AJR. American journal of roentgenology

Brain stimulation
Count

3

3

\begin{tabular}{lr}
\hline Journal & Count \\
\hline Journal of surgical research & 3 \\
Digestion & 3 \\
Human brain mapping & 3 \\
Journal of clinical densitometry: the official journal of the & \\
International Society for Clinical Densitometry & 3 \\
Ophthalmic and physiological optics: the journal of the &
\end{tabular}

British College of Ophthalmic Opticians (Optometrists)

Journal of infection

Blood pressure. Supplement

British journal of psychology (London, England: 1953) 3

European journal of oral sciences

Langenbeck's archives of surgery/Deutsche Gesellschaft für 3 Chirurgie

Breast journal

Clinical journal of sport medicine: official journal of the 3 Canadian Academy of Sport Medicine

Indian journal of dermatology, venereology and leprology 3

Journal of cerebral blood flow and metabolism: official 3 journal of the International Society of Cerebral Blood Flow and Metabolism

International journal of cosmetic science 3

Annals of neurology 3

Journal of the National Cancer Institute. Monographs 3

Prostate cancer and prostatic diseases 3

Congestive heart failure (Greenwich, Conn.) 3

Nature neuroscience 3

CNS neuroscience and therapeutics 3

Chinese medical sciences journal $=$ Chung-kuo i hsüeh k’o 3 hsüeh tsa chih/Chinese Academy of Medical Sciences

Leukemia and lymphoma 3

Asia-Pacific journal of clinical oncology 3

Obesity research 3

Virology journal 3

Melanoma research 3

Circulation research $\quad 3$

Biomedical chromatography: BMC 3

International endodontic journal 3

Social psychiatry and psychiatric epidemiology 3

American journal of health behavior 3

European journal of surgical oncology: the journal of the 3 European Society of Surgical Oncology and the British Association of Surgical Oncology

Journal of hypertension. Supplement: official journal of the 3 International Society of Hypertension

Journal of nervous and mental disease $\quad 3$

British journal of radiology 3

Acta tropica 3

Inflammation research: official journal of the European 3 Histamine Research Society... [et al.]

Photodermatology, photoimmunology and photomedicine 3 


Journal
AMIA... Annual Symposium proceedings/AMIA
Symposium. AMIA Symposium
Food and chemical toxicology: an international journal
published for the British Industrial Biological Research
Association

Scandinavian journal of rheumatology

Studies in health technology and informatics

Journal of heart and lung transplantation: the official publication of the International Society for Heart

Transplantation

Amino acids

Biomarkers: biochemical indicators of exposure, response, and susceptibility to chemicals

Annals of emergency medicine

British journal of clinical practice

Clinical neurophysiology: official journal of the International

Federation of Clinical Neurophysiology

Infection and immunity

Academic emergency medicine: official journal of the

Society for Academic Emergency Medicine

Lipids

Psychology of addictive behaviors: journal of the Society of

Psychologists in Addictive Behaviors

Journal of ethnopharmacology

International journal of hematology

Alcohol and alcoholism (Oxford, Oxfordshire)

Archives of sexual behavior

Journal of obesity

American journal of public health

European journal of pharmaceutics and biopharmaceutics: official journal of Arbeitsgemeinschaft für

Pharmazeutische Verfahrenstechnik e.V

Digestive and liver disease: official journal of the Italian Society of Gastroenterology and the Italian Association for the Study of the Liver

Influenza and other respiratory viruses

Methods and findings in experimental and clinical pharmacology

American journal of physiology. Renal physiology

American journal of clinical oncology

Die Pharmazie

Shock (Augusta, Ga.)

Nephron. Clinical practice

Journal of neuro-oncology

Journal of experimental psychology. Learning, memory, and cognition

CNS drugs

Journal of physiology

International journal of cardiovascular imaging

Pediatric surgery international

Ophthalmic epidemiology

Wiener klinische Wochenschrift
Count

3

3

3

3

\begin{tabular}{ll}
\hline Journal & Count \\
\hline
\end{tabular}

International archives of occupational and environmental health

Advances in health sciences education: theory and practice 3

Cognition 3

Acta cardiologica 3

Journal of science and medicine in sport/Sports Medicine 3 Australia

Journal of physiological anthropology 3

Asian journal of andrology 3

Archives of oral biology 3

Indian journal of dental research: official publication of 3 Indian Society for Dental Research

Journal of medicinal chemistry 3

Xenobiotica; the fate of foreign compounds in biological 3 systems

Drug safety 3

Developmental medicine and child neurology 3

Life sciences $\quad 3$

Aesthetic plastic surgery 3

Journal of laparoendoscopic and advanced surgical 3 techniques. Part A

Cancer immunology, immunotherapy: CII 3

Addiction science and clinical practice 3

Radiology 3

Disability and rehabilitation $\quad 3$

Pediatric emergency care $\quad 3$

Cell metabolism 3

Pediatric dermatology 3

European journal of internal medicine 3

Diseases of the colon and rectum 3

Archives of gerontology and geriatrics 3

Journal of shoulder and elbow surgery/American Shoulder 3 and Elbow Surgeons... [et al.]

Medical teacher 3

International journal of obstetric anesthesia 3

Alzheimer's and dementia: the journal of the Alzheimer's 3 Association

Journal of critical care $\quad 3$

Renal failure 3

American journal of physiology. Heart and circulatory 3 physiology

Journal of biomedicine and biotechnology 3

PLoS pathogens 3

Cancer nursing $\quad 3$

American journal of emergency medicine 3

Pediatric exercise science $\quad 3$

Oral oncology 2

International journal of environmental research and public 2 health

Expert opinion on drug metabolism and toxicology 2 


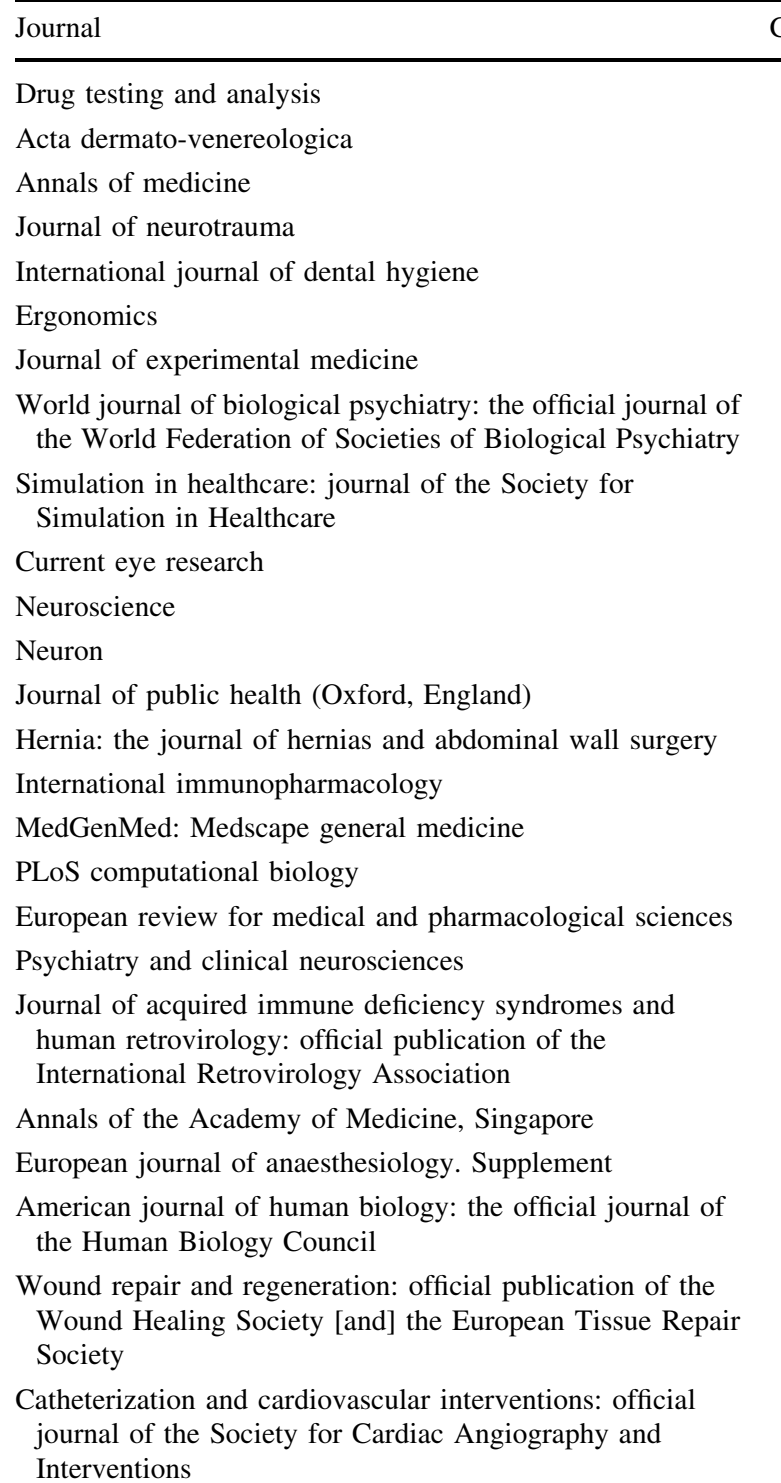

International journal of neuroscience

Annals of cardiac anaesthesia

Endocrine journal

Journal of andrology

Neurosciences (Riyadh, Saudi Arabia)

American journal of drug and alcohol abuse

Psychosomatics

Primary care respiratory journal: journal of the General

Practice Airways Group

Journal of abnormal psychology

Drugs under experimental and clinical research

Journal of athletic training

Nursing times

Journal of psychosomatic obstetrics and gynaecology

Obesity facts

Clinical colorectal cancer

Journal of dermatology
Count

2

2

2

2

2

2

2

2

2

2

2

2

2

2

2

2

2

2

2

2

2

2

2

2

2

\begin{tabular}{ll}
\hline Journal & Count \\
\hline
\end{tabular}

International journal for vitamin and nutrition research.

Internationale Zeitschrift für Vitamin- und

Ernährungsforschung. Journal international de

vitaminologie et de nutrition

Pediatric obesity 2

Journal of the National Medical Association 2

Journal of health communication 2

Journal of cutaneous medicine and surgery 2

Child psychiatry and human development 2

Burns: journal of the International Society for Burn Injuries 2

Southern medical journal 2

Prevention science: the official journal of the Society for 2

Prevention Research

Ocular immunology and inflammation 2

Caries research 2

Canadian journal of gastroenterology $=$ Journal canadien de 2 gastroenterologie

Memory (Hove, England) 2

Cancer immunity 2

Journal of the American Podiatric Medical Association 2

Paediatrics and international child health 2

International journal of oral science 2

Danish medical journal 2

Journal of investigational allergology and clinical 2 immunology

Journal of laryngology and otology 2

British journal of clinical practice. Supplement 2

Chirality 2

Motor control 2

Diabetes educator $\quad 2$

Journal of back and musculoskeletal rehabilitation 2

Journal of medical screening $\quad 2$

Reproductive health $\quad 2$

Chronic respiratory disease $\quad 2$

Australian and New Zealand journal of public health 2

Journal of laboratory and clinical medicine 2

Journal of neuro-ophthalmology: the official journal of the 2

North American Neuro-Ophthalmology Society

Optometry and vision science: official publication of the 2

American Academy of Optometry

Quintessence international (Berlin, Germany: 1985) 2

Acta odontologica Scandinavica 2

Agents and actions. Supplements 2

Canadian journal of psychiatry. Revue canadienne de 2 psychiatrie

Journal of inherited metabolic disease $\quad 2$

Zeitschrift für Gastroenterologie $\quad 2$

Transactions of the American Clinical and Climatological 2

Association 


Journal
Journal of population therapeutics and clinical pharmacology
= Journal de la thérapeutique des populations et de la
pharamcologie clinique
Korean journal of internal medicine
Pan African medical journal
Journal of obstetrics and gynaecology: the journal of the
Institute of Obstetrics and Gynaecology
Nouvelle revue française d'hématologie
Cognitive science
Journal of sports medicine and physical fitness
Croatian medical journal
Hormones (Athens, Greece)
Academic pediatrics
Translational research: the journal of laboratory and clinical
medicine

Psychiatric rehabilitation journal

Respiratory care

Journal of clinical psychology

International journal of adolescent medicine and health

Coronary artery disease

Immunopharmacology

Nucleosides, nucleotides and nucleic acids

Journal of obstetrics and gynaecology research

Treatments in respiratory medicine

Manual therapy

Heart disease (Hagerstown, Md.)

Endocrine

Oncology (Williston Park, NY)

European journal of oncology nursing: the official journal of European Oncology Nursing Society

Journal of wound care

Taiwanese journal of obstetrics and gynecology

Hormones and behavior

Surgery

Alternative therapies in health and medicine

Therapeutic apheresis and dialysis: official peer-reviewed journal of the International Society for Apheresis, the Japanese Society for Apheresis, the Japanese Society for Dialysis Therapy

Kidney international. Supplement

Research in sports medicine (Print)

Journal of reproductive medicine

Journal of obstetrics and gynaecology Canada

Expert review of anticancer therapy

Thyroid: official journal of the American Thyroid Association

European journal of health economics: HEPAC: health economics in prevention and care

Circulation. Heart failure

Journal of burn care and research: official publication of the American Burn Association
Count

\begin{tabular}{lc}
\hline Journal Count \\
\hline
\end{tabular}

Hormone research in paediatrics

Pharmacoepidemiology and drug safety 2

Skinmed $\quad 2$

Free radical biology and medicine $\quad 2$

Canadian journal of surgery. Journal canadien de chirurgie 2

Nature medicine $\quad 2$

BMC medical imaging $\quad 2$

Applied nursing research: ANR 2

Aging clinical and experimental research 2

International journal of eating disorders 2

Research in developmental disabilities $\quad 2$

Oral health and preventive dentistry 2

International journal for quality in health care: journal of the 2 International Society for Quality in Health Care/ISQua

Neonatology 2

Journal of tropical pediatrics 2

Liver transplantation: official publication of the American 2 Association for the Study of Liver Diseases and the International Liver Transplantation Society

Journal of rheumatology. Supplement 2

Amyotrophic lateral sclerosis: official publication of the 2 World Federation of Neurology Research Group on Motor Neuron Diseases

British journal of rheumatology 2

Pakistan journal of biological sciences: PJBS 2

Cleveland Clinic journal of medicine 2

Diabetes, nutrition and metabolism 2

General hospital psychiatry 2

Proceedings of the Nutrition Society 2

Scandinavian cardiovascular journal: SCJ 2

Zhong xi yi jie he xue bao $=$ Journal of Chinese integrative 2 medicine

Vascular and endovascular surgery 2

Social cognitive and affective neuroscience 2

Carcinogenesis 2

Italian journal of pediatrics $\quad 2$

Journal of vascular and interventional radiology: JVIR 2

Clinical chemistry 2

Canadian family physician Médecin de famille canadien 2

Diseases of the esophagus: official journal of the 2

International Society for Diseases of the Esophagus/I.S.D.E

Journal of aging and physical activity 2

Journal of medical virology 2

West African journal of medicine 2

Japanese journal of ophthalmology 2

International journal of rheumatic diseases 2

Journal of infection and chemotherapy: official journal of the 2 Japan Society of Chemotherapy

Brain research 2

Journal of adhesive dentistry 2 


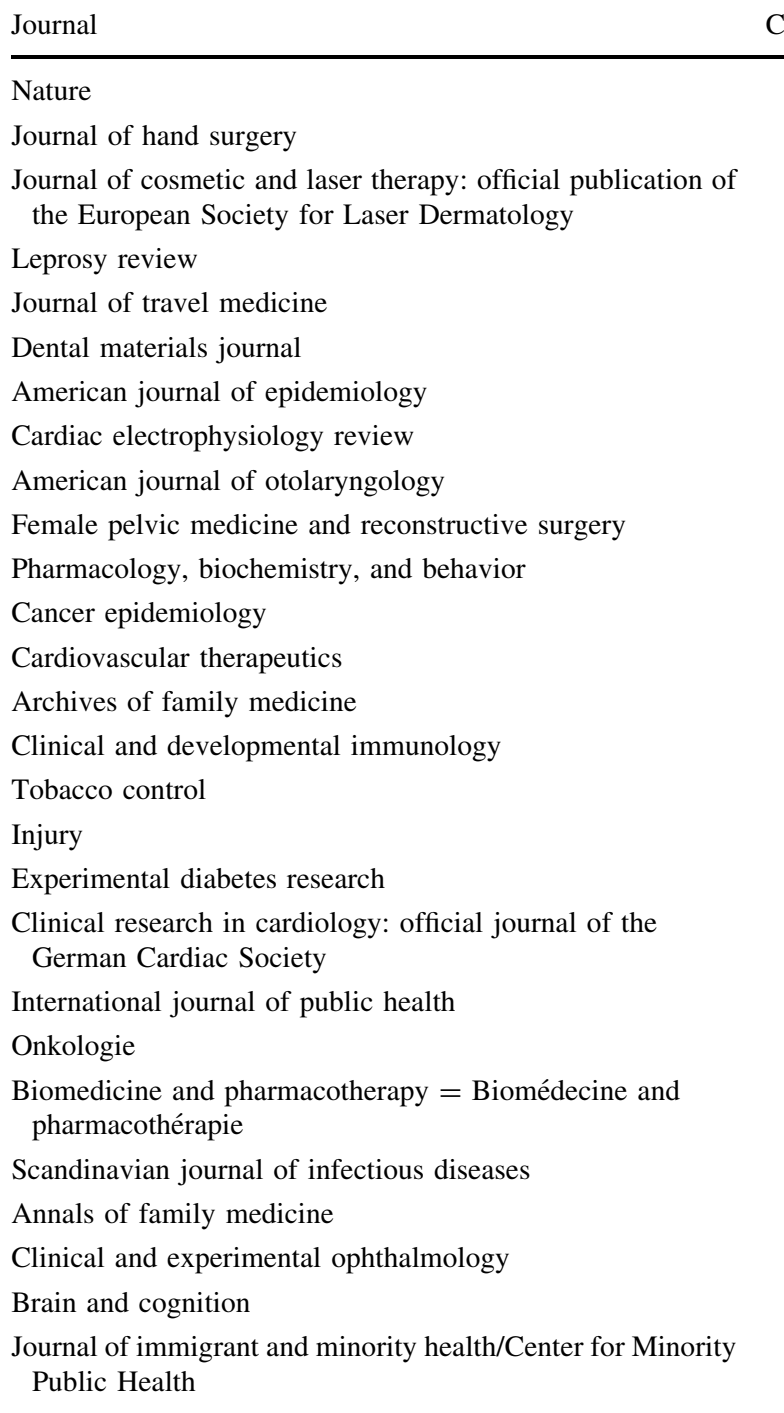

Primary care diabetes

Orphanet journal of rare diseases

Clinical and experimental dermatology

Acta neurologica Scandinavica

Drug metabolism and pharmacokinetics

American journal of clinical dermatology

Archives of disease in childhood. Fetal and neonatal edition

Annals of otology, rhinology, and laryngology

Acta obstetricia et gynecologica Scandinavica

Experimental dermatology

American journal of community psychology

Transplant infectious disease: an official journal of the

Transplantation Society

International journal of fertility and women's medicine

Acta physiologica (Oxford, England)

Clinical neurology and neurosurgery

Health economics

Drugs and aging

Women's health (London, England)
Count

2

2

2

2

2

2

2

2

2

2

2

2

2

2

2

2

2

2

2

\begin{tabular}{lr}
\hline Journal & Count \\
\hline International journal of immunopathology and pharmacology & 2 \\
JAMA psychiatry & 2 \\
Neuroepidemiology & 2 \\
BMC physiology & 2 \\
Mycoses & 2 \\
Journal of community health & 2 \\
Pituitary & 2 \\
International journal of pediatric obesity: IJPO: an official & 2 \\
journal of the International Association for the Study of & \\
Obesity & 2 \\
Expert review of clinical immunology & 2 \\
Journal of interferon and cytokine research: the official &
\end{tabular}
journal of the International Society for Interferon and Cytokine Research

Journal of chromatography. B, Biomedical sciences and 2 applications

Psychology and aging

British journal of social psychology/the British Psychological Society

Controlled clinical trials

European journal of physical and rehabilitation medicine

Digestive endoscopy: official journal of the Japan Gastroenterological Endoscopy Society

Archives of women's mental health

Cornea

European addiction research

Journal of Crohn's and colitis

JPEN. Journal of parenteral and enteral nutrition

Annals of noninvasive electrocardiology: the official journal of the International Society for Holter and Noninvasive Electrocardiology, Inc

British journal of oral and maxillofacial surgery 2

Ear, nose, and throat journal 2

Clinical respiratory journal 2

Psychological science $\quad 2$

Prostate 2

European journal of cardiovascular nursing: journal of the 2 Working Group on Cardiovascular Nursing of the European Society of Cardiology

Journal of clinical virology: the official publication of the 2 Pan American Society for Clinical Virology

Free radical research 2

Journal of the International AIDS Society 2

Clinical and translational oncology: official publication of 2 the Federation of Spanish Oncology Societies and of the National Cancer Institute of Mexico

Journal of interventional cardiac electrophysiology: an 2 international journal of arrhythmias and pacing

South African medical journal $=$ Suid-Afrikaanse tydskrif $\quad 2$ vir geneeskunde

Journal of clinical pediatric dentistry 2

Seminars in hematology 2 


\begin{tabular}{l} 
Journal \\
\hline BMC microbiology \\
Inhalation toxicology \\
Journal of psychiatric and mental health nursing \\
Journal of pharmaceutical and biomedical analysis \\
Director (Cincinnati, Ohio) \\
Eye and contact lens \\
Future oncology (London, England) \\
Journal of cancer research and clinical oncology \\
American journal of orthodontics and dentofacial \\
orthopedics: official publication of the American \\
Association of Orthodontists, its constituent societies, and \\
the American Board of Orthodontics \\
Clinical nephrology \\
Behavioural brain research \\
Journal of renal nutrition: the official journal of the Council \\
on Renal Nutrition of the National Kidney Foundation \\
European journal of public health \\
Surgery, gynecology and obstetrics \\
Journal of electromyography and kinesiology: official journal \\
of the International Society of Electrophysiological \\
Kinesiology
\end{tabular}

Pancreas

International journal of STD and AIDS

Explore (New York, NY)

Pharmacological research: the official journal of the Italian Pharmacological Society

Expert review of cardiovascular therapy

Therapeutic advances in respiratory disease

Archives of andrology

Australian journal of rural health

Issues in mental health nursing

Australian dental journal

Acta cirúrgica brasileira/Sociedade Brasileira para

Desenvolvimento Pesquisa em Cirurgia

Journal of medicine and life

Skin pharmacology and applied skin physiology

Minimally invasive therapy and allied technologies: MITAT:

official journal of the Society for Minimally Invasive

Therapy

Journal of pediatric psychology

Inflammation and allergy drug targets

Journal of the American College of Surgeons

Medical education online

Nature genetics

Annals of human biology

Journal of neuroscience methods

Archives of cardiovascular diseases

Urology journal

Journal of health care for the poor and underserved

Immunology letters

ANZ journal of surgery
Count

2

2

2

2

2

2

2

2

2

2

2

2

2

2

2

2

2

2

2

2

2

2

2

1

1

1

1

1

1

1

1

1

1

1

1

1
Journal

Count

Swiss medical weekly

International journal of circumpolar health

Journal of molecular biology

Annals of the Royal College of Surgeons of England

American journal of physiology. Regulatory, integrative and comparative physiology

Developmental neurorehabilitation de recherche en sciences infirmières

Journal of medical microbiology

Korean journal of hepatology

PM \& R: the journal of injury, function, and rehabilitation

Journal of the American Osteopathic Association

Journal of otolaryngology-head and neck surgery = Le Journal d'oto-rhino-laryngologie et de chirurgie cervicofaciale

Journal of radiological protection: official journal of the Society for Radiological Protection

Surgery for obesity and related diseases: official journal of the American Society for Bariatric Surgery

Aging

Japan journal of nursing science: JJNS

Developmental science

Body image

Journal of women's health/the official publication of the Society for the Advancement of Women's Health Research

Journal of the cardiometabolic syndrome

Journal of perianesthesia nursing: official journal of the American Society of PeriAnesthesia Nurses/American Society of PeriAnesthesia Nurses

International journal of obesity

Journal of obstetric, gynecologic, and neonatal nursing: JOGNN/NAACOG

Strahlentherapie und Onkologie: Organ der Deutschen 1 Röntgengesellschaft ... [et al.]

Journal of cardiovascular medicine (Hagerstown, Md.) 1

Neuroradiology

Virus research

Journal of invasive cardiology

Bratislavské lekárske listy

Ultrasound in medicine and biology 1

Anxiety, stress, and coping

Cyberpsychology and behavior: the impact of the Internet, multimedia and virtual reality on behavior and society

Dental materials: official publication of the Academy of Dental Materials

Journal of sex and marital therapy

Journal of the renin-angiotensin-aldosterone system: JRAAS

Journal of the College of Physicians and Surgeons-Pakistan: JCPSP

Regenerative medicine

Journal of palliative medicine 


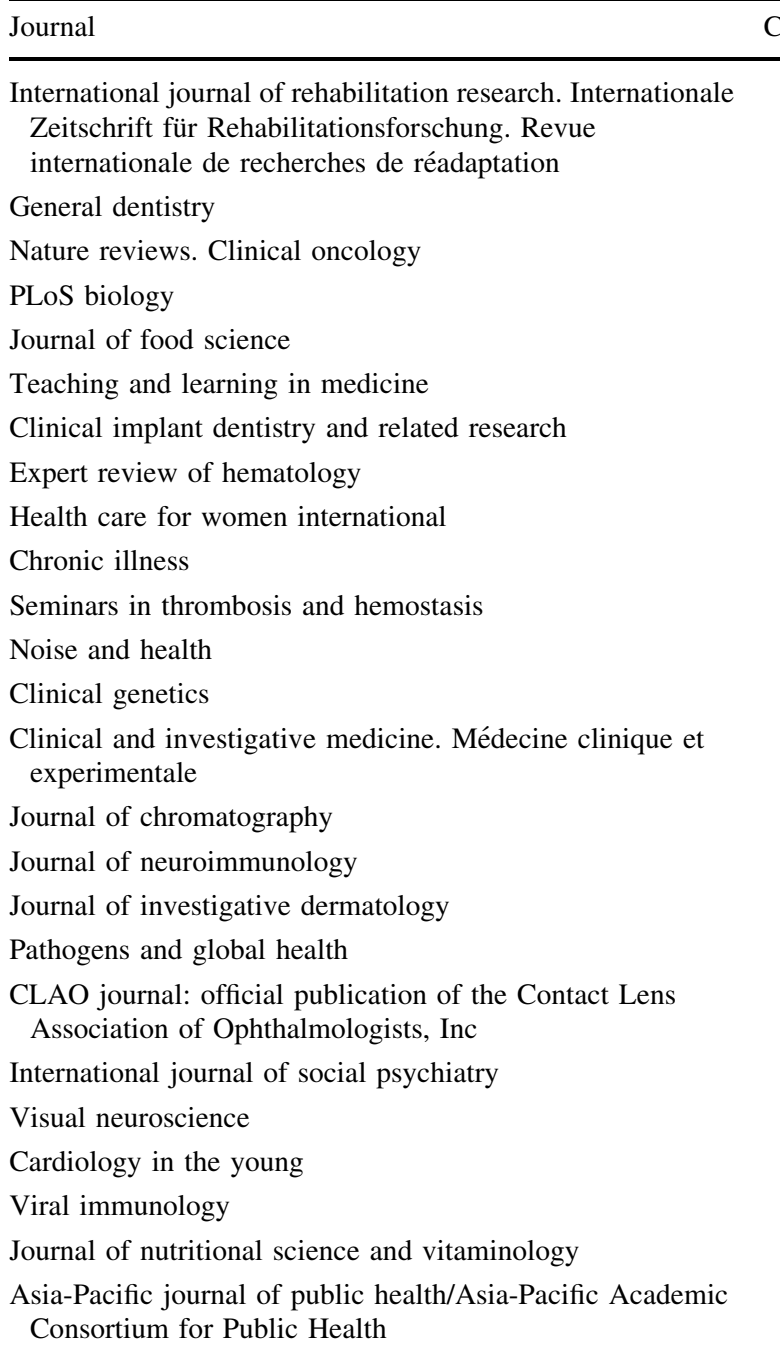

Journal of electrocardiology

São Paulo medical journal $=$ Revista paulista de medicina

Spinal cord

Cancer investigation

Circulation. Cardiovascular imaging

Wiener medizinische Wochenschrift (1946)

Acta paediatrica Scandinavica. Supplement

Pediatrics and neonatology

Brazilian oral research

Archives of physiology and biochemistry

Vox sanguinis

Medical engineering and physics

Digestive surgery

PLoS genetics

Nitric oxide: biology and chemistry/official journal of the Nitric Oxide Society

Canadian journal of clinical pharmacology $=$ Journal canadien de pharmacologie clinique

QJM: monthly journal of the Association of Physicians
Count

1

Surgical laparoscopy, endoscopy and percutaneous techniques

Journal of tissue viability

Australian journal of primary health

Clinical oral implants research

Clinical imaging

Aging (Milan, Italy)

Heart and vessels

Restorative neurology and neuroscience

Actas españolas de psiquiatría

Asian cardiovascular and thoracic annals

Progress in community health partnerships: research, education, and action

Future microbiology

Canadian journal of cardiovascular nursing $=$ Journal canadien en soins infirmiers cardio-vasculaires

Journal of virological methods

Pediatric transplantation

Clinical prostate cancer

Theoretical biology and medical modelling

Journal of neurointerventional surgery

Molecular imaging and biology: MIB: the official publication 1 of the Academy of Molecular Imaging

Odontology/the Society of the Nippon Dental University 1

Current opinion in HIV and AIDS 1

Expert review of respiratory medicine 1

Journal of oral science 1

International journal of sports physiology and performance 1

BMC medical genomics 1

Acta chirurgica Belgica 1

Journal of voice: official journal of the Voice Foundation 1

BioMed research international 1

Pediatric neurology 1

Orthopedic nursing 1

Journal of dental education $\quad 1$

American journal of human genetics 1

Virology 1

Epidemiology (Cambridge, Mass.) 1

Physiotherapy research international: the journal for $\quad 1$ researchers and clinicians in physical therapy

Clinical and experimental immunology 1

Psicothema 1

International journal of medical informatics 1

Asian journal of surgery/Asian Surgical Association $\quad 1$

Behavioural processes 1

Inflammation 1

Journal of pharmacological sciences 1

Drug and alcohol review 1

Magma (New York, NY) 1

Education for health (Abingdon, England) 1

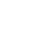
1 1 1 1 


Journal Count

Journal of orthopaedic research: official publication of the Orthopaedic Research Society

European journal of nuclear medicine

Cerebellum (London, England)

International surgery

Journal of nutritional biochemistry

Journal of perinatal medicine

Cell stress and chaperones

Somatosensory and motor research

Cell biochemistry and biophysics

International journal of clinical pharmacy

Tumour biology: the journal of the International Society for Oncodevelopmental Biology and Medicine

Acta neurochirurgica

Archives of pathology and laboratory medicine

Journal of orthopaedic science: official journal of the

Japanese Orthopaedic Association

Tuberculosis (Edinburgh, Scotland)

American review of respiratory disease

Journal of trace elements in medicine and biology: organ of the Society for Minerals and Trace Elements (GMS)

Journal of health economics

European journal of esthetic dentistry: official journal of the European Academy of Esthetic Dentistry

Personality and social psychology bulletin

Early human development

Zentralblatt für Gynäkologie

Nutrition and cancer

Nutritional neuroscience

Child development

Experimental and toxicologic pathology: official journal of the Gesellschaft für Toxikologische Pathologie

Reviews of infectious diseases

Journal of periodontal research

Health promotion international

Journal of rehabilitation research and development

Quality in health care: QHC

Internal medicine journal

Chronobiology international

Economics and human biology

Kurume medical journal

Journal of trauma

Phlebology/Venous Forum of the Royal Society of Medicine

Annali italiani di chirurgia

Documenta ophthalmologica. Advances in ophthalmology

Neuro endocrinology letters

Annals of human genetics

Eating and weight disorders: EWD

Journal of postgraduate medicine

Journal of vestibular research: equilibrium and orientation

\begin{tabular}{lc}
\hline Journal Count \\
\hline
\end{tabular}

Drug development and industrial pharmacy

International journal of clinical practice. Supplement

Reviews on recent clinical trials

Medicine and sport science

Oncology nursing forum

Journal of psychiatric practice

Sexual health

Promotion and education

International journal of pediatric otorhinolaryngology

Journal of psycholinguistic research

Journal of urban health: bulletin of the New York Academy of Medicine

Acta haematologica

GMS Zeitschrift für medizinische Ausbildung

Revista panamericana de salud pública $=$ Pan American journal of public health

Journal of communication disorders

Brain and language

Eating behaviors

Statistical methods in medical research

Contributions to nephrology

British heart journal

Joint Commission journal on quality improvement

European archives of paediatric dentistry: official journal of the European Academy of Paediatric Dentistry

Journal of neuroscience nursing: journal of the American Association of Neuroscience Nurses

Psychotherapy (Chicago, Ill.)

Urological research

Quality and safety in health care

Angiology

Behavior therapy

Dysphagia

Expert opinion on drug delivery

Arthritis care and research: the official journal of the Arthritis Health Professions Association

Compendium of continuing education in dentistry. (Jamesburg, NJ: 1995). Supplement

Journals of gerontology. Series B, Psychological sciences and social sciences

Behavioural and cognitive psychotherapy

Physician and sportsmedicine

Experimental cell research

Nigerian journal of medicine: journal of the National Association of Resident Doctors of Nigeria

Journal of nuclear cardiology: official publication of the American Society of Nuclear Cardiology

International journal of oncology

Journal of hand surgery, European volume

Epileptic disorders: international epilepsy journal with videotape 


Journal
Journal of immunoassay
Otology and neurotology: official publication of the
American Otological Society, American Neurotology
Society [and] European Academy of Otology and
Neurotology

Aviation, space, and environmental medicine

Journal of pain and palliative care pharmacotherapy

Molecular cancer therapeutics

Gerontology

Neurological sciences: official journal of the Italian

Neurological Society and of the Italian Society of Clinical

Neurophysiology

Toxicology and applied pharmacology

Tissue antigens

Culture, health and sexuality

Gastroentérologie clinique et biologique

Clinical and experimental nephrology

European journal of general practice

British dental journal

Journal of adolescence

Molecular pain

American journal of medical quality: the official journal of the American College of Medical Quality

Maternal and child nutrition

Acta myologica: myopathies and cardiomyopathies: official journal of the Mediterranean Society of Myology/edited by the Gaetano Conte Academy for the study of striated muscle diseases

Bulletin of Tokyo Dental College

American journal on addictions/American Academy of Psychiatrists in Alcoholism and Addictions

Journal of clinical child and adolescent psychology: the official journal for the Society of Clinical Child and Adolescent Psychology, American Psychological Association, Division 53

Molecular medicine (Cambridge, Mass.)

Transfusion and apheresis science: official journal of the World Apheresis Association: official journal of the

European Society for Haemapheresis

Journal of molecular neuroscience: MN

Musculoskeletal care

Journal of the Association for Research in Otolaryngology: JARO

Physiotherapy

Journal of immunological methods

Evaluation review

Current drug delivery

Journal of the Acoustical Society of America

International journal of biological sciences

Current cardiology reports

Herz

Journal of biomechanics

Count

1

1

1

1

1

1

1

1

1

1

1

1

1

1

1

1

1

\section{Journal}

Count

Statistical applications in genetics and molecular biology

International orthodontics/Collège européen d'orthodontie

Journal of clinical investigation

Zeitschrift für Rheumatologie

International journal for equity in health

Journal of applied microbiology

Journal of forensic sciences

La Clinica terapeutica

Journal of wound, ostomy, and continence nursing: official publication of the Wound, Ostomy and Continence Nurses Society/WOCN

Journal of health services research and policy

Journal of minimally invasive gynecology

Annals of hematology

BMC immunology

Orthopaedics and traumatology, surgery and research: OTSR

Current opinion in investigational drugs (London, England: 2000)

Journal of neurosurgery

Irish journal of medical science

Cognitive, affective and behavioral neuroscience

Current opinion in pharmacology

Journal of microbiology, immunology, and infection = Wei mian yu gan ran za zhi

Brain research bulletin

Saudi journal of kidney diseases and transplantation: an official publication of the Saudi Center for Organ Transplantation, Saudi Arabia

Journal of nuclear medicine: official publication, Society of Nuclear Medicine

Journal of dietary supplements 1

Journal of Ayub Medical College, Abbottabad: JAMC 1

Haemophilia: the official journal of the World Federation of 1 Hemophilia

Scandinavian journal of surgery: SJS: official organ for the Finnish Surgical Society and the Scandinavian Surgical Society

Journal of clinical and experimental neuropsychology

Ambulatory pediatrics: the official journal of the Ambulatory Pediatric Association

Journal of pharmacological and toxicological methods $\quad 1$

Proceedings. Biological sciences/the Royal Society 1

Neurocritical care

Biochemical pharmacology

Anxiety

Nature clinical practice. Cardiovascular medicine

Journal of vector borne diseases

Journal of epidemiology and biostatistics

Academic medicine: journal of the Association of American Medical Colleges

Blood transfusion $=$ Trasfusione del sangue 


\section{Journal \\ JACC. Cardiovascular imaging \\ Journal of health psychology \\ Journal of traumatic stress \\ Indian journal of cancer \\ Indian journal of pharmacology \\ Cancer discovery \\ Addiction biology}

Count

Health promotion journal of Australia: official journal of Australian Association of Health Promotion Professionals

Pediatric critical care medicine: a journal of the Society of Critical Care Medicine and the World Federation of Pediatric Intensive and Critical Care Societies

Clinical and experimental optometry: journal of the

Australian Optometrical Association

Journal of pediatric and adolescent gynecology

Journal of integrative medicine

Occupational medicine (Oxford, England)

Marine drugs

Annals of anatomy $=$ Anatomischer Anzeiger: official organ of the Anatomische Gesellschaft

German medical science: GMS e-journal

American journal of the medical sciences

Nordic journal of psychiatry

European journal of cancer prevention: the official journal of the European Cancer Prevention Organisation (ECP)

Pediatric blood and cancer

Physiotherapy theory and practice

European journal of echocardiography: the journal of the

Working Group on Echocardiography of the European

Society of Cardiology

Journal of acupuncture and meridian studies

Journal of Zhejiang University. Science. B

Journal of neuroinflammation

European journal of paediatric dentistry: official journal of European Academy of Paediatric Dentistry

Annals of ophthalmology

Health and place

Academic radiology

Drugs of today (Barcelona, Spain: 1998)

African journal of medicine and medical sciences

Minerva stomatologica

International journal of food sciences and nutrition

Rejuvenation research

Journal of cancer survivorship: research and practice

Substance use and misuse

Food and nutrition bulletin

Journal of applied biomechanics

Australian endodontic journal: the journal of the Australian Society of Endodontology Inc

Paediatric and perinatal epidemiology

Journal of musculoskeletal and neuronal interactions

\begin{tabular}{lc}
\hline Journal Count \\
\hline
\end{tabular}

Journal of managed care pharmacy: JMCP

Liver international: official journal of the International 1 Association for the Study of the Liver

Head and neck

Journal of refractive surgery (Thorofare, NJ: 1995)

Biological trace element research

Acta medica Iranica

Current biology: $\mathrm{CB}$

Mental health services research

1

Perfusion

Journal of clinical monitoring and computing

Kidney and blood pressure research

Biometrics

JAMA internal medicine

SADJ: journal of the South African Dental

Association = tydskrif van die Suid-Afrikaanse

Tandheelkundige Vereniging

International journal of language and communication disorders/Royal College of Speech and Language Therapists

Emerging infectious diseases

Journal of visualized experiments: JoVE

Heart and lung: the journal of critical care

1

Public health

Archives of Iranian medicine

Hospital practice (1995)

International journal of clinical and experimental pathology

Parasitology research

Biological research for nursing

Journal of pregnancy

Clinical and experimental medicine

Bulletin of the World Health Organization

Clinical psychology and psychotherapy

Immunopharmacology and immunotoxicology

Archives of virology

Journal of maternal-fetal and neonatal medicine: the official journal of the European Association of Perinatal Medicine, the Federation of Asia and Oceania Perinatal Societies, the International Society of Perinatal Obstetricians

Australasian journal of dermatology

Vascular medicine (London, England)

International journal on drug policy

Journal of orofacial pain

British journal of community nursing

Journal of rural health: official journal of the American Rural Health Association and the National Rural Health Care Association

Anesthesia progress

Journal of preventive medicine and hygiene

Preventing chronic disease

Journal of craniofacial surgery 


\begin{tabular}{|c|c|}
\hline Journal & Coun \\
\hline Indian journal of physiology and pharmacology & 1 \\
\hline Canadian journal of urology & 1 \\
\hline Experimental psychology & 1 \\
\hline $\begin{array}{l}\text { Journal of family psychology: JFP: journal of the Division of } \\
\text { Family Psychology of the American Psychological } \\
\text { Association (Division 43) }\end{array}$ & 1 \\
\hline European journal of epidemiology & 1 \\
\hline Behavior modification & 1 \\
\hline Research quarterly for exercise and sport & 1 \\
\hline Endocrine-related cancer & 1 \\
\hline Journal of the American Optometric Association & 1 \\
\hline Cancer science & 1 \\
\hline Journal of steroid biochemistry and molecular biology & 1 \\
\hline Journal of pediatric ophthalmology and strabismus & 1 \\
\hline Blood reviews & 1 \\
\hline Scandinavian journal of immunology & 1 \\
\hline International nursing review & 1 \\
\hline International journal of audiology & 1 \\
\hline [Nihon koshu eisei zasshi] Japanese journal of public health & 1 \\
\hline Journal of emergency medicine & 1 \\
\hline $\begin{array}{l}\text { Journal of neurological surgery. Part A, Central European } \\
\text { neurosurgery }\end{array}$ & 1 \\
\hline Western journal of nursing research & 1 \\
\hline $\begin{array}{l}\text { Eastern Mediterranean health journal = La revue de santé de } \\
\text { la Méditerranée orientale = al-Majallah al-ṣị̣hịyah li-sharq } \\
\text { al-mutawassit }\end{array}$ & 1 \\
\hline American journal of medical genetics. Part A & 1 \\
\hline $\begin{array}{l}\text { Hip international: the journal of clinical and experimental } \\
\text { research on hip pathology and therapy }\end{array}$ & 1 \\
\hline Urologic clinics of North America & 1 \\
\hline $\begin{array}{l}\text { Ophthalmic surgery, lasers and imaging: the official journal } \\
\text { of the International Society for Imaging in the Eye }\end{array}$ & 1 \\
\hline Middle East African journal of ophthalmology & 1 \\
\hline Parasites and vectors & 1 \\
\hline Nigerian journal of clinical practice & 1 \\
\hline Canadian journal of physiology and pharmacology & 1 \\
\hline Perceptual and motor skills & 1 \\
\hline Iranian journal of kidney diseases & 1 \\
\hline Molecular cancer & 1 \\
\hline Topics in stroke rehabilitation & 1 \\
\hline Frontiers of radiation therapy and oncology & 1 \\
\hline Evaluation and the health professions & 1 \\
\hline Comprehensive psychiatry & 1 \\
\hline IEEE transactions on biomedical engineering & 1 \\
\hline Pharmacy world and science: PWS & 1 \\
\hline International journal of andrology & 1 \\
\hline Drug research & 1 \\
\hline New Zealand medical journal & 1 \\
\hline Journal of learning disabilities & 1 \\
\hline Clinical neuroradiology & 1 \\
\hline
\end{tabular}


Journal

Count

Food and function

Journal of cardiology

Neuroscience research

Amyotrophic lateral sclerosis and other motor neuron disorders: official publication of the World Federation of Neurology, Research Group on Motor Neuron Diseases

Indian journal of gastroenterology: official journal of the Indian Society of Gastroenterology

Journal of agricultural and food chemistry

Child abuse and neglect

Annals of transplantation: quarterly of the Polish

Transplantation Society

Journal of geriatric psychiatry and neurology

Current atherosclerosis reports

Future cardiology

Acta oto-laryngologica

Hormone and metabolic research. Supplement series

Journal of palliative care

Medical archives (Sarajevo, Bosnia and Herzegovina)

European journal of neuroscience

Australian family physician

Bosnian journal of basic medical sciences/Udruženje basičnih mediciniskih znanosti $=$ Association of Basic Medical Sciences

Culture, medicine and psychiatry

Journal of clinical rheumatology: practical reports on rheumatic and musculoskeletal diseases

Medical mycology

Child neuropsychology: a journal on normal and abnormal development in childhood and adolescence

International journal of biological markers

Human genomics

Gut microbes

Neurotoxicology

Rhinology

Journal of agricultural safety and health

Journal of human nutrition and dietetics: the official journal of the British Dietetic Association

International journal of periodontics and restorative dentistry

Journal of cardiovascular surgery

Journal of physiology and pharmacology: an official journal of the Polish Physiological Society

Chemico-biological interactions

Journal of clinical pathology

Tropical gastroenterology: official journal of the Digestive Diseases Foundation

Dermatologica

American journal of Chinese medicine

Molecular aspects of medicine

Seizure

Behavioral neuroscience

1

1

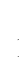

1

1

1

1

1

1

\begin{tabular}{lc}
\hline Journal Count \\
\hline
\end{tabular}

Alcohol research and health: the journal of the National

Institute on Alcohol Abuse and Alcoholism

International braz $\mathrm{j}$ urol: official journal of the Brazilian

Society of Urology

British journal of neurosurgery

Acta neurologica Scandinavica. Supplementum

Journal of psychosocial oncology

Journal of child and adolescent psychiatric nursing: official 1 publication of the Association of Child and Adolescent

Psychiatric Nurses, Inc

Journal of investigative and clinical dentistry 1

Medicine

Journal of surgical oncology

Medical microbiology and immunology

Clinical biochemistry

Neurology India

Skin therapy letter

International journal of tuberculosis and lung disease: the official journal of the International Union against

Tuberculosis and Lung Disease

Minerva chirurgica

Biochemia medica

Neuromolecular medicine

Scandinavian journal of psychology

Acta radiologica (Stockholm, Sweden: 1987)

Urologic oncology

Work (Reading, Mass.)

American journal of perinatology

Community mental health journal

American journal of physiology. Lung cellular and molecular physiology

Psychonomic bulletin and review

Genome biology

Cardiology journal

Current clinical pharmacology

European journal of histochemistry: EJH 1

New York state dental journal 1

Nephron

Cancer journal (Sudbury, Mass.)

International journal of urology: official journal of the 1 Japanese Urological Association

Scandinavian cardiovascular journal. Supplement 1

Cognitive processing 1

Medical principles and practice: international journal of the 1 Kuwait University, Health Science Centre

Journal of hospital infection 1

Cytokine 1

Evolutionary psychology: an international journal of $\quad 1$ evolutionary approaches to psychology and behavior

Journal of the Pakistan Medical Association 
Journal

Count

Pulmonary pharmacology

Hemodialysis international. International Symposium on

Home Hemodialysis

Experimental neurology

Seminars in liver disease

Neurotoxicity research

Danish medical bulletin

Attention deficit and hyperactivity disorders

Lung

\section{Hippocampus}

Journal of personality and social psychology

Clinical immunology (Orlando, Fla.)

International journal of surgery (London, England)

Acta ophthalmologica Scandinavica

Journal of chromatography. B, Analytical technologies in the biomedical and life sciences

Haematology and blood transfusion

Acta bio-medica: Atenei Parmensis

Gastric cancer: official journal of the International Gastric

Cancer Association and the Japanese Gastric Cancer

Association

Enfermedades infecciosas y microbiología clínica

Journal of neural transmission. Parkinson's disease and dementia section

Clinical cornerstone

Journal of interpersonal violence

Pain management nursing: official journal of the American Society of Pain Management Nurses

Breast (Edinburgh, Scotland)

Journal of developmental and behavioral pediatrics: JDBP

International angiology: a journal of the International Union of Angiology

Archives of otolaryngology-head and neck surgery

Neural plasticity

Journal of cardiopulmonary rehabilitation

International ophthalmology

Biomedical engineering online

Nursing ethics

Bioscience, biotechnology, and biochemistry

Gastroenterology nursing: the official journal of the Society of Gastroenterology Nurses and Associates

Dermatitis: contact, atopic, occupational, drug

Telemedicine journal and e-health: the official journal of the American Telemedicine Association

Acta otorhinolaryngologica Italica: organo ufficiale della Società italiana di otorinolaringologia e chirurgia cervicofacciale

1

1

1

1

1

1

1

1

1

1

1

1
Appendix 4: List of adjectives considered relevant and number of articles in which they were used

$$
\text { Word }
$$
Count

\section{Significant}

Similar

Effective

Important

Severe

Major

Moderate

Long-term

Comparable

Mild

Serious

Safe

Consistent

Usual

Relevant

Good

Conventional $\quad 537$

Complete 493

Poor 471

Potent 435

Useful $\quad 432$

Appropriate $\quad 355$

Apparent $\quad 350$

Efficacious $\quad 347$

Meaningful $\quad 345$

Intensive $\quad 345$

Substantial $\quad 337$

Unchanged $\quad 337$

Successful $\quad 337$

Improved $\quad 325$

Acceptable $\quad 315$

Necessary $\quad 295$

Well tolerated 287

Favorable 279

Strong 271

Modest 257

Novel 256

Adequate 241

Promising 240

Validated $\quad 235$

Simple $\quad 229$

Minor $\quad 225$

Equal 224

Difficult 223

Traditional 215 


\begin{tabular}{|c|c|c|c|}
\hline Word & Count & Word & Coun \\
\hline Feasible & 210 & Thorough & 56 \\
\hline Essential & 207 & Established & 56 \\
\hline Cost-effective & 198 & Preferred & 55 \\
\hline Protective & 192 & Numerous & 55 \\
\hline Inadequate & 190 & Formal & 54 \\
\hline Enhanced & 189 & Crucial & 54 \\
\hline Evident & 185 & Marginal & 54 \\
\hline Comprehensive & 175 & Prominent & 53 \\
\hline Reliable & 168 & Inconsistent & 52 \\
\hline Sufficient & 166 & Viable & 52 \\
\hline Evidence-based & 155 & Widespread & 51 \\
\hline Robust & 155 & Negligible & 50 \\
\hline Unlikely & 146 & Strongest & 49 \\
\hline Efficient & 144 & Reasonable & 49 \\
\hline Excellent & 140 & Harmful & 49 \\
\hline Mild-to-moderate & 131 & Notable & 49 \\
\hline Extensive & 130 & Inconclusive & 49 \\
\hline Unexpected & 129 & Pivotal & 48 \\
\hline Considerable & 128 & Expensive & 48 \\
\hline Valid & 124 & Detrimental & 47 \\
\hline Faster & 111 & Unnecessary & 47 \\
\hline Favourable & 110 & Definite & 46 \\
\hline Stronger & 108 & Superficial & 46 \\
\hline Fast & 105 & Putative & 46 \\
\hline Detectable & 104 & Ineffective & 46 \\
\hline Inferior & 102 & Hard & 45 \\
\hline Responsible & 101 & Profound & 44 \\
\hline Valuable & 98 & Precise & 44 \\
\hline Pronounced & 94 & Inexpensive & 44 \\
\hline Non-significant & 87 & Insignificant & 44 \\
\hline Definitive & 83 & Urgent & 44 \\
\hline Weak & 82 & Desirable & 43 \\
\hline Conservative & 82 & Ideal & 42 \\
\hline Excessive & 82 & Probable & 42 \\
\hline Controversial & 78 & Problematic & 42 \\
\hline Poorer & 77 & Sustainable & 41 \\
\hline Uncertain & 76 & Non-specific & 41 \\
\hline Suboptimal & 75 & Vigorous & 39 \\
\hline Satisfactory & 74 & Inappropriate & 39 \\
\hline Non-significant & 74 & Long-lasting & 38 \\
\hline Easy & 72 & Unacceptable & 38 \\
\hline Convenient & 69 & Extreme & 35 \\
\hline Correct & 69 & Unfavorable & 34 \\
\hline Theoretical & 68 & Non-specific & 34 \\
\hline Empirical & 67 & Synergistic & 34 \\
\hline Costly & 63 & Theory-based & 34 \\
\hline Attractive & 61 & Challenging & 33 \\
\hline Tolerable & 57 & Obvious & 33 \\
\hline
\end{tabular}




\begin{tabular}{|c|c|c|c|}
\hline Word & Count & Word & Coun \\
\hline Unhealthy & 32 & Generalizable & 16 \\
\hline Classical & 32 & Unsuitable & 16 \\
\hline Fundamental & 32 & In-depth & 16 \\
\hline Classic & 30 & Next-generation & 16 \\
\hline Powerful & 30 & Demonstrable & 16 \\
\hline Universal & 30 & Affordable & 16 \\
\hline Interesting & 30 & Holistic & 16 \\
\hline Nominal & 30 & Worthwhile & 16 \\
\hline Encouraging & 29 & Discordant & 15 \\
\hline Low-risk & 28 & Ultimate & 15 \\
\hline Suspected & 28 & Life-threatening & 15 \\
\hline Well known & 28 & Appreciable & 15 \\
\hline Opportunistic & 26 & Perfect & 15 \\
\hline Safer & 25 & Unfavourable & 14 \\
\hline Submaximal & 25 & Striking & 14 \\
\hline Plausible & 24 & Debilitating & 14 \\
\hline Confirmatory & 24 & Inspired & 13 \\
\hline Preferable & 24 & Noticeable & 13 \\
\hline Diminished & 24 & Unusual & 13 \\
\hline Easier & 23 & Unresolved & 12 \\
\hline Fair & 23 & Enormous & 12 \\
\hline Advantageous & 23 & Credible & 12 \\
\hline Weaker & 22 & Compelling & 12 \\
\hline Suggestive & 22 & Rewarding & 12 \\
\hline Informative & 22 & Contrary & 12 \\
\hline Unsuccessful & 22 & Fastest & 12 \\
\hline Remarkable & 21 & Well accepted & 12 \\
\hline Risky & 21 & Troublesome & 11 \\
\hline Unexplained & 21 & Noteworthy & 11 \\
\hline Conclusive & 21 & Pleasant & 11 \\
\hline Proven & 20 & Impossible & 11 \\
\hline Disadvantaged & 19 & Unbiased & 11 \\
\hline High-quality & 19 & Subtle & 11 \\
\hline Preferential & 19 & Logical & 11 \\
\hline Stringent & 19 & Unwanted & 11 \\
\hline Hazardous & 19 & Threatening & 11 \\
\hline Convincing & 18 & Irrelevant & 11 \\
\hline Dramatic & 18 & $\mathrm{Bad}$ & 10 \\
\hline Incorrect & 18 & Integrative & 10 \\
\hline Well established & 18 & Full-scale & 10 \\
\hline Devastating & 17 & Presumed & 10 \\
\hline Unpleasant & 17 & Failed & 10 \\
\hline Confident & 17 & Milder & 10 \\
\hline Very & 17 & Disruptive & 10 \\
\hline Intolerable & 17 & Enough & 10 \\
\hline Undesirable & 17 & Vast & 10 \\
\hline Disappointing & 16 & Advisable & 9 \\
\hline Counterbalanced & 16 & Surprising & 9 \\
\hline
\end{tabular}




\begin{tabular}{|c|c|}
\hline Word & Coun \\
\hline Intriguing & 9 \\
\hline Substantive & 9 \\
\hline Empiric & 9 \\
\hline Strategic & 9 \\
\hline Discernible & 9 \\
\hline Sub-optimal & 9 \\
\hline Elusive & 9 \\
\hline Conflicting & 9 \\
\hline Simpler & 9 \\
\hline Imperative & 9 \\
\hline Long-term & 9 \\
\hline Well documented & 8 \\
\hline Genuine & 8 \\
\hline Moral & 8 \\
\hline Inadvertent & 8 \\
\hline Nonserious & 8 \\
\hline Instructional & 8 \\
\hline Exaggerated & 8 \\
\hline Non-serious & 7 \\
\hline Intuitive & 7 \\
\hline Provocative & 7 \\
\hline Doubtful & 7 \\
\hline Disproportionate & 7 \\
\hline Concrete & 7 \\
\hline Pervasive & 7 \\
\hline Enjoyable & 7 \\
\hline Contradictory & 7 \\
\hline Gentle & 7 \\
\hline Inaccurate & 7 \\
\hline Unanticipated & 7 \\
\hline Unreliable & 6 \\
\hline Cost-saving & 6 \\
\hline Pertinent & 6 \\
\hline Consensus-based & 6 \\
\hline Exciting & 6 \\
\hline Debatable & 6 \\
\hline Under-represented & 6 \\
\hline Unsafe & 6 \\
\hline Understudied & 6 \\
\hline Questionable & 6 \\
\hline Sophisticated & 6 \\
\hline Well characterized & 6 \\
\hline Competent & 6 \\
\hline Tremendous & 6 \\
\hline Mild-moderate & 6 \\
\hline Big & 6 \\
\hline Provisional & 6 \\
\hline Subnormal & 5 \\
\hline
\end{tabular}

\begin{tabular}{lr}
\hline Word & Count \\
\hline Generalisable & 5 \\
Verified & 5 \\
Infinite & 5 \\
Sizeable & 5 \\
Undesired & 5 \\
Objectively & 5 \\
Inefficient & 5 \\
Instructive & 5 \\
Uncomfortable & 5 \\
Usable & 5 \\
Confirmative & 5 \\
Seminal & 4 \\
Weakly & 4 \\
\hline
\end{tabular}

\section{References}

1. Dickersin K. The existence of publication bias and risk factors for its occurrence. JAMA. 1990;263(10):1385-9.

2. Easterbrook PJ, Berlin JA, Gopalan R, Matthews DR. Publication bias in clinical research. Lancet. 1991;337(8746):867-72.

3. Schulz KF, Altman DG, Moher D. CONSORT 2010 statement: updated guidelines for reporting parallel group randomized trials. Ann Intern Med. 2010;152(11):726-32.

4. Han C, Kwak KP, Marks DM, Pae CU, Wu LT, Bhatia KS, et al. The impact of the CONSORT statement on reporting of randomized clinical trials in psychiatry. Contemp Clin Trials. 2009;30(2):116-22.

5. Alvarez F, Meyer N, Gourraud PA, Paul C. CONSORT adoption and quality of reporting of randomized controlled trials: a systematic analysis in two dermatology journals. Br J Dermatol. 2009;161(5):1159-65.

6. De AC, Drazen JM, Frizelle FA, Haug C, Hoey J, Horton R, et al. Clinical trial registration: a statement from the International Committee of Medical Journal Editors. N Engl J Med. 2004;351(12):1250-1.

7. Vera-Badillo FE, Shapiro R, Ocana A, Amir E, Tannock IF. Bias in reporting of end points of efficacy and toxicity in randomized, clinical trials for women with breast cancer. Ann Oncol. 2013;24(5):1238-44.

8. Torvik VI, Smalheiser NR. Author name disambiguation in Medline. ACM Trans Knowl Discov Data. 2009;3(3). pii: 11.

9. Breiman L. Random forests. Mach Learn. 2001;45:5-32.

10. The Apache Software Foundation. OpenNLP. The Apache Software Foundation. http://opennlp.apache.org/index.html. 2010. Cited 28 Oct 2013.

11. Penn Treebank Project. Alphabetical list of part-of-speech tags used in the Penn Treebank Project. https://www.ling.upenn. edu/courses/Fall_2003/ling001/penn_treebank_pos.html. 2015. Accessed 20 Feb 2015

12. Ferraro JP, Daume H III, Duvall SL, Chapman WW, Harkema H, Haug PJ. Improving performance of natural language processing 
part-of-speech tagging on clinical narratives through domain adaptation. J Am Med Inform Assoc. 2013;20(5):931-9.

13. Martin DO, Austin H. An exact method for meta-analysis of casecontrol and follow-up studies. Epidemiology. 2000;11(3):255-60.

14. Holm S. A simple sequentally rejective multiple test procedure. Scand J Stat. 1979;6(2):65-70.

15. Mansi BA, Clark J, David FS, Gesell TM, Glasser S, Gonzalez J, et al. Ten recommendations for closing the credibility gap in reporting industry-sponsored clinical research: a joint journal and pharmaceutical industry perspective. Mayo Clin Proc. 2012;87(5):424-9.
16. van LM, Overbeke J, Out HJ. Recommendations for a uniform assessment of publication bias related to funding source. BMC Med Res Methodol. 2013; 13(1):120.

17. Schwartz RS, Curfman GD, Morrissey S, Drazen JM. Full disclosure and the funding of biomedical research. N Engl J Med. 2008;358(17):1850-1.

18. Boutron I, Altman DG, Hopewell S, Vera-Badillo F, Tannock I, Ravaud P. Impact of spin in the abstracts of articles reporting results of randomized controlled trials in the field of cancer: the SPIIN randomized controlled trial. J Clin Oncol. 2014;32(36):4120-6. 